Multiscale Simulations

of

Star Polymer Melts

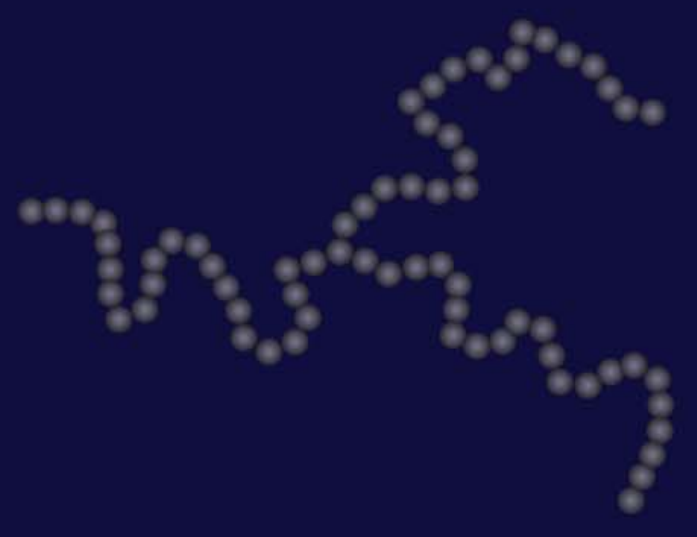

Li Liu 
MULTISCALE SIMULATIONS OF STAR POLYMER MELTS

LI LIU 
Promotion committee:

Prof. dr. H. Hilgenkamp University of Twente (Chairman)

Prof. dr. H. Hilgenkamp University of Twente (Secretary)

Prof. dr. W. J. Briels University of Twente (Supervisor)

Dr. ir. W. K. den Otter University of Twente (Ass. Supervisor)

Prof. dr. S. Luding University of Twente

Dr. H. T. M. van den Ende University of Twente

Prof. dr. ir. J. van der Gucht Wageningen University, The Netherlands

Prof. dr. D. Vlassopoulos University of Crete, Greece

Prof. dr. J. K. G. Dhont Forschungszentrum Jülich, Germany

\section{Liu Li}

Multiscale simulations of star polymer melts

Ph.D. Thesis, University of Twente, Enschede

This project is supported with a grant of the European Union's Seventh Framework Programme (FP7) through the Marie Curie initial training network DYNamics of Architecturally Complex Polymers (DYNACOP) and the European Soft Matter Infrastructure (ESMI).

ISBN: 978-90-365-3686-8

Copyright (c) Li Liu, Enschede, The Netherlands, 2014. All rights reserved. DOI: $10.3990 / 1.9789036536868$

Online version: http://dx.doi.org/10.3990/1.9789036536868

Typeset in ATE $_{\mathrm{E}} \mathrm{X}$ by the author

Front cover: schematic of a star at different scales, by Shuwen Yu

Back cover photos: Seasons@UTwente, Matenweg

Printed by: Gildeprint Drukkerijen - www.gildeprint.nl 


\title{
MULTISCALE SIMULATIONS OF STAR POLYMER MELTS
}

\author{
DISSERTATION
}

to obtain

the degree of doctor at the University of Twente,

on the authority of the rector magnificus,

prof. dr. H. Brinksma,

on account of the decision of the graduation committee,

to be publicly defended

on Friday, 20 June 2014 at 16:45

by

\section{Li Liu}

born on 4 November 1981

in Liaoning, China 
This dissertation has been approved by:

Prof. dr. W. J. Briels (promoter)

and

Dr. W. K. den Otter (assistant promoter) 
How is life? 



\section{Contents}

1 Introduction 1

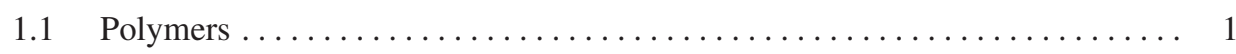

1.1.1 What is a polymer? ..........................

1.1.2 Polymer Architectures . . . . . . . . . . . . . . . 1

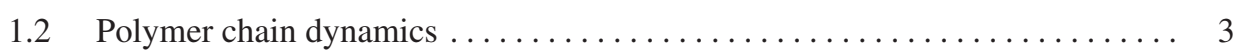

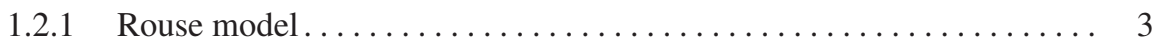

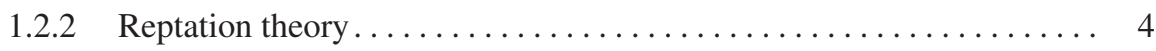

1.3 Particle-based computer simulations $\ldots \ldots \ldots \ldots \ldots \ldots \ldots \ldots \ldots \ldots$

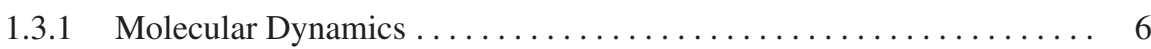

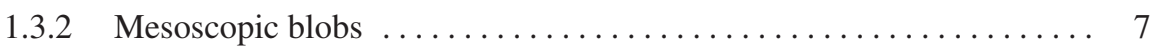

1.3.3 Coarse-Graining to a Single Particle $\ldots \ldots \ldots \ldots \ldots \ldots \ldots \ldots$

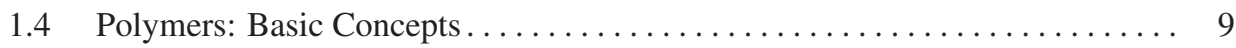

1.4.1 End-to-End Distance and Radius of Gyration. . . . . . . . . . . . 9

1.4.2 Mean Square Displacement and Diffusivity . . . . . . . . . . . 10

1.4.3 Stress autocorrelation and Viscoelasticity ............... 10

1.5 Thesis outline $\ldots \ldots \ldots \ldots \ldots \ldots \ldots \ldots \ldots \ldots \ldots \ldots \ldots \ldots \ldots \ldots$

2 Coarse-grained Simulations of Moderately Entangled Star Polyethylene Melt 13

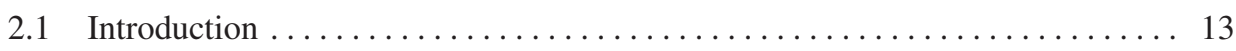

2.2 Model overview and Simulated systems $\ldots \ldots \ldots \ldots \ldots \ldots \ldots \ldots \ldots$

2.2.1 Coarse-graining method and Brownian dynamics . . . . . . . . 15

2.2.2 Uncrossability constraint $\ldots \ldots \ldots \ldots \ldots \ldots \ldots \ldots \ldots \ldots \ldots \ldots \ldots \ldots \ldots \ldots \ldots$

2.2.3 Topology specification and simulation details $\ldots \ldots \ldots \ldots \ldots \ldots$

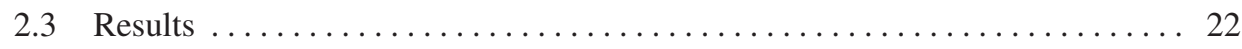

2.3.1 Dynamics and rheological properties of star polymer $\ldots \ldots \ldots \ldots 24$

2.3.2 Comparisons between star and linear polymers .............. 29 


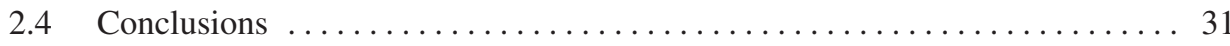

Appendix : Rouse model of nonlinear polymers ................... 32

3 Simulations of Symmetric Star Polymers with Different Arm Lengths 37

3.1 Introduction . . . . . . . . . . . . . . . . . . . . . . . . . . . . 37

3.2 Model and methods $\ldots \ldots \ldots \ldots \ldots \ldots \ldots \ldots \ldots \ldots \ldots \ldots \ldots \ldots$

3.2.1 Coarse grained polymers .......................... 40

3.2.2 Uncrossability constraints ......................... 41

3.2 .3 Analysis methods .............................. 43

3.3 Results and Discussion $\ldots \ldots \ldots \ldots \ldots \ldots \ldots \ldots \ldots \ldots \ldots \ldots \ldots \ldots$

$3.3 .1 \quad$ Linear polymers $\ldots \ldots \ldots \ldots \ldots \ldots \ldots \ldots \ldots \ldots \ldots \ldots \ldots \ldots$

3.3.2 Three-armed stars $\ldots \ldots \ldots \ldots \ldots \ldots \ldots \ldots \ldots \ldots \ldots \ldots \ldots, 47$

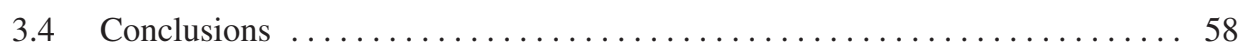

Appendix : Rouse relaxation modulus $\ldots \ldots \ldots \ldots \ldots \ldots \ldots \ldots \ldots \ldots \ldots$

4 Dynamics of Asymmetric Star Polymers under Coarse Grain Simulations 63

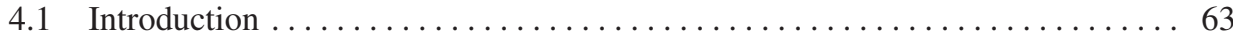

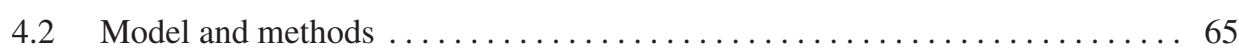

4.2.1 Simulation model................................ 65

4.2.2 Analysis methods ............................... 67

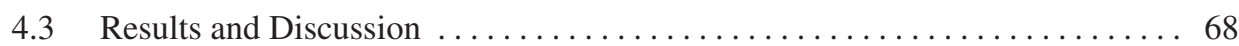

$4.3 .1 \quad$ T-shaped polymers $\ldots \ldots \ldots \ldots \ldots \ldots \ldots \ldots \ldots \ldots \ldots \ldots \ldots \ldots$

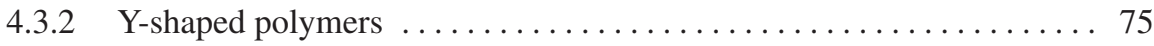

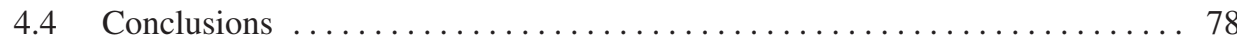

5 Coarse Grain Forces in Star Polymer Melts 79

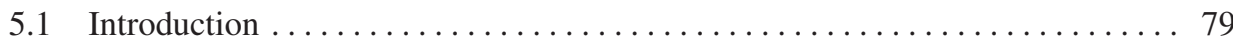

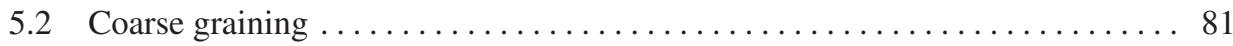

5.2.1 Potential of mean force $\ldots \ldots \ldots \ldots \ldots \ldots \ldots \ldots \ldots \ldots \ldots$

$5.2 .2 \quad$ Transient forces $\ldots \ldots \ldots \ldots \ldots \ldots \ldots \ldots \ldots \ldots \ldots \ldots \ldots \ldots \ldots \ldots$

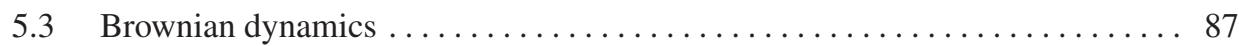

5.4 Coarse parameters from small scale simulations . . . . . . . . . . . . . . 89 
CONTENTS

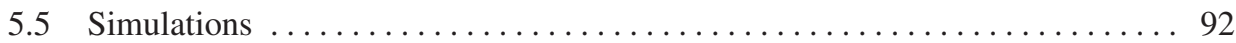

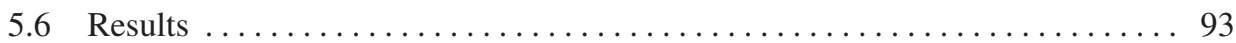

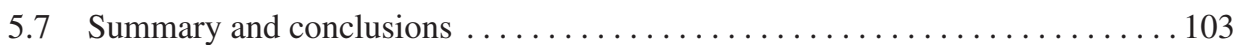

$\begin{array}{ll}\text { Summary } & 107\end{array}$

Samenvatting $\quad 111$

$\begin{array}{ll}\text { Acknowledgment } & 115\end{array}$

$\begin{array}{ll}\text { List of publications } & 117\end{array}$

$\begin{array}{lc}\text { Bibliography } & 118\end{array}$ 



\section{Introduction}

\subsection{Polymers}

\subsubsection{What is a polymer?}

Polymers are very much commonplace in the modern world, from natural rubber to familiar synthetics such as acrylic, nylon, and different kinds of plastics, etcetera [1,2]. They are of particular interest to industry due to their broad range of applications and one of the most fascinating materials for academic investigation. So what exactly is a polymer?

By definition, a polymer is a large molecule built from many covalently bonded units (monomers). Based on the identity of those repeated monomers, polymers are generally termed into homo or hetero groups. When all of the monomers are of the same type, the polymer is called a homopolymer while a polymer comprising of blocks with different chemical constitutions is referred to as a heteropolymer.

\subsubsection{Polymer Architectures}

Due to its simple structure and associated thermoplastic feature, polyethylene (PE) represents one of the most frequently used polymers in industry [3]. Depending on its density and degree of branching, PE can be used in the manufacture of many products such as plastic packaging, human joint replacement components and for water and gas piping. PE is a long chain polymer composed of carbon and hydrogen atoms arranged as shown in Fig. 1.1. It can be melted to a liquid-like state that can be re-molded to form a variety of different forms before returning to a solid state upon cooling.

The physical properties of polymers such as PE are strongly correlated to their underlying 


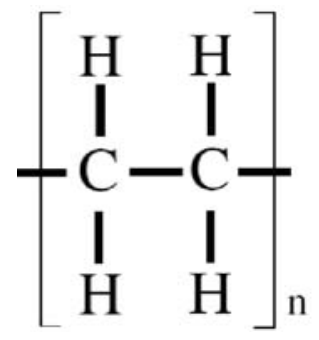

Figure 1.1: Polyethylene monomer.

architectures [4-11]. In general PE architectures can be divided into two categories: linear or branched. Linear PE facilitates the close packing of adjacent chains and is thus referred to as high density polyethylene (HDPE). On the other hand, branched PE is characterized by the number of branching points and the method of linking. The degree of branching significantly affects the close packing of chains and thus branched PE can be referred to as low density polyethylene (LDPE). The simplest branched polymer type is a star polymer which consists of a number of linear polymers covalently bonded to a single branching point. Star polymer properties are dependent upon the number of linear polymers (or arms) as well as the molecular mass of each arm [12-19]. Other varieties of branched polymers include $\mathrm{H}-$ type polymers which consist of two branching points, comb-like polymers where a number of chains are attached to a backbone chain with two or more chains at each branching point, and dendritic polymers with a very high degree of branching [20-24]. Examples of these branching architectures are shown in Fig. 1.2.

In this thesis, we will study the dynamic and rheological properties for the simplest branched polymer architecture. Therefore we will focus on homopolymers with a star architecture under melt conditions. For a fundamental understanding and comparison with star polymer melts, we will study linear polymer melts to ascertain the importance of branching on their rheological response. 


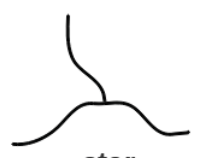

star

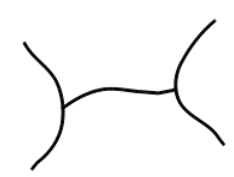

H-typed

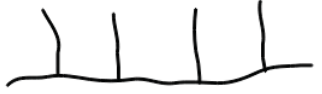

Comb-like

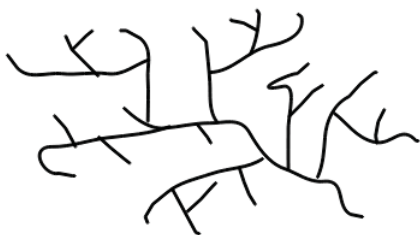

Dendritic polymer

Figure 1.2: Schematic presentation of different branched polymer architectures.

\subsection{Polymer chain dynamics}

The properties of linear polymer chains has been subject of extensive studies. From these studies it is known that the dynamics of linear polymers are dependent on the chain length [18, 25-33]. Short chain dynamics can be described using the Rouse model [34]. With increasing chain length, the mobility of the polymers decrease due to the development of interpolymer entanglements. For long chain polymers their behaviours are influenced by topological interactions which drastically affect the dynamic properties of the chains. Long chain polymer behaviors can be described by Reptation Theory $[35,36]$.

\subsubsection{Rouse model}

In the Rouse model, each polymer is subdivided into Gaussian segments connected together by harmonic springs as illustrated in Fig. 1.3. In effect the Rouse model is a bead-spring representation of a polymer chain where each bead is not subject to excluded volume effects and the hydrodynamic interactions between the beads are ignored. As a result the motion of a chain is caused by a friction force associated with the intra-molecular interactions and an additional stochastic or random force.

The Rouse model is applicable to short linear chains in the melt state. It correctly predicts the scaling of the center of mass diffusion constant where $D \sim N^{-1}$ and demonstrates that the 


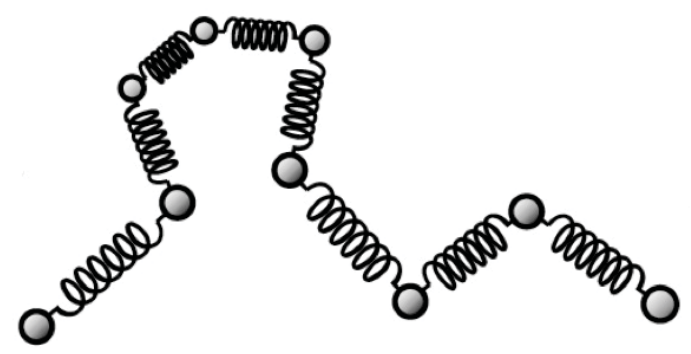

Figure 1.3: A polymer chain in bead-spring model. (Adapted from [37])

viscosity is proportional to the chain length of an unentangled polymer. However, it does not yield the correct expressions for slow diffusion behavior or the large relaxation time for melts consisting of high molecular weight linear polymers where entanglement effects dominate.

\subsubsection{Reptation theory}

The slow motion of long highly entangled polymer chains cannot be entirely captured by the Rouse model which is applicable up to a crossover time $\tau_{e}$. To predict chain dynamics beyond this crossover, Doi and Edwards refined reptation theory [35], introduced by de Gennes [36], by making it applicable to highly entangled polymer liquids. For a polymer melt, chains cannot pass through each other due to their elastic property. Each polymer is subject to topological constraints from surrounding chains. These constraints are referred to as entanglements and can be represented by a tube within which the polymer performs a snake-like motion as shown in Fig. 1.4. For a single reptating chain, the theory predicts that the viscosity and longest relaxation time follow the scaling law of $N^{3}$, while the diffusion constant scales as $D \sim N^{-2}$ where $N$ is the polymer length.

\subsection{Particle-based computer simulations}

As the third branch of scientific study, computer simulations have been become more and more prominent with advancements in modern computing technologies [38,39]. They offer increased control over system parameters in comparison to experiments, access to measures 


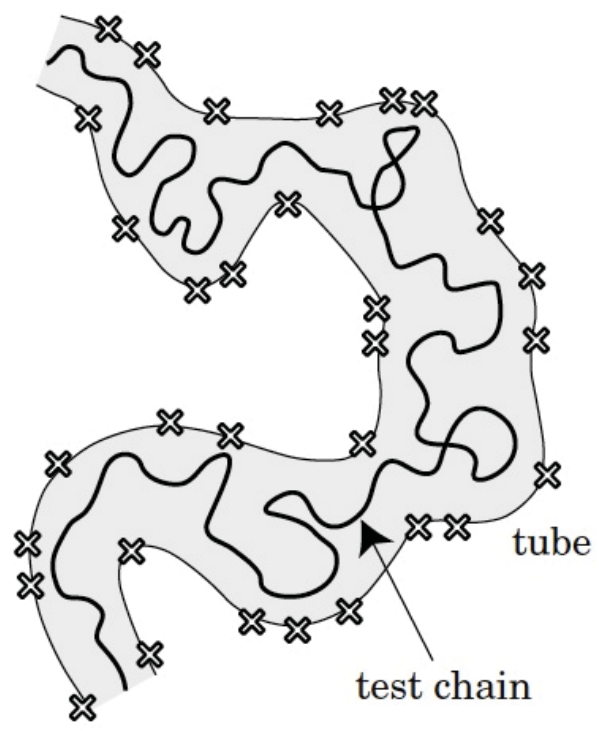

Figure 1.4: A test chain reptating in a tube defined by entanglements(crossings) with surrounding chains.(Adapted from [37])

sometimes inaccessible in experiments and a platform to test theoretical predictions.

At first sight, it is tempting to perform a polymer simulation where all chemical structures are included in an effort to capture as accurately as possible all interaction details. However, polymers exhibit clear time and length scaling in their dynamical behaviors. A direct prediction of the large scale dynamics and rheology from such a detailed method would require a huge computational effort that would prove costly in terms of computation time. To reduce the computational cost for the simulation of polymeric systems, coarse-graining approaches may be applied for the study of rheological responses in such systems [40-42]. The central concept in coarse graining approaches is the representation a group of atoms or monomers as a larger group unit that neglects the atomistic details within the unit. By this means, the replaced realistic parts will interact with each other as new repeated coarse grained particles. Fig. 1.5 shows a schematic representation of a linear chain under atomistic level and subject to different coarse graining degrees.

The evolution of the particle dynamics does not depend on the level of coarse graining 


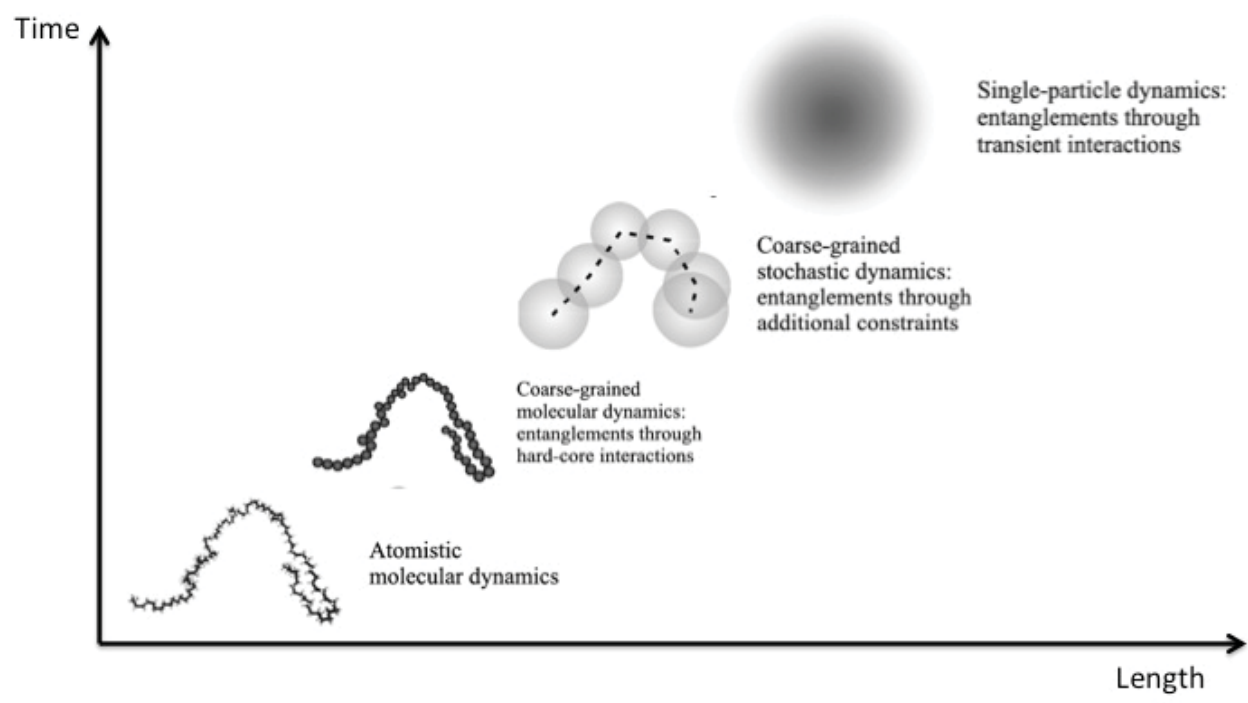

Figure 1.5: Schematic representation of different levels of coarse-graining for a polymer chain.(Adapted from [41])

applied to the constituent particles. For particles confined to a box with periodic boundary conditions, the forces on the particles are firstly calculated. Using these forces, the positions and the velocities (if a 2nd order integrator is used) are then updated for the next time step. This process then continues for the duration of the program.

\subsubsection{Molecular Dynamics}

In coarse-grained molecular simulations, one particle represents the center of mass of a few atoms. Accordingly a polymer chain will be substituted with a string of hard spheres or beads with a sufficient repulsive potential at short distance to prevent chain crossings. One of the most used approaches for the simulation of such a system is the Molecular Dynamics (MD) algorithm. In MD particle positions and velocities are evolved by integrating Newton's laws of motion once the force on each particle has been calculated. Typically MD simulations can only be applied to very short times as a small integration time step is required in order to attain 
a stable solution. In their MD simulations of polymer melts, Kremer and Grest (1990) [43] implemented a standard bead-spring coarse-grained approach where two beads from any two polymer chains interact via the repulsive Lennard-Jones pair potential,

$$
U_{i j}^{r e p}=\left\{\begin{array}{cl}
4 \varepsilon\left[\left(\frac{\sigma}{r_{i j}}\right)^{12}-\left(\frac{\sigma}{r_{i j}}\right)^{6}+\frac{1}{4}\right], & r_{i j} \leq 2^{1 / 6} \sigma \\
0, & r_{i j}>2^{1 / 6} \sigma
\end{array}\right.
$$

where $r_{i j}$ is the vector separating the two beads, $\sigma$ is an effective diameter and $\varepsilon$ is the well depth. In addition, the bond between two adjacent bonds in a chain was allowed to deform subject to a FENE (Finite-Extensible Non-Linear Elastic) potential,

$$
U_{i j}^{a t t}=\left\{\begin{array}{cc}
-0.5 k R_{0}^{2} \ln \left[1-\left(\frac{r_{i j}}{R_{0}}\right)^{2}\right], & r_{i j} \leq R_{0} \\
\infty, & r_{i j}>R_{0}
\end{array}\right.
$$

where $k$ is the spring constant and $R_{0}$ is the maximum bond length. The FENE potential provides a penalty on the free energy upon deformation. In their simulations, Kremer and Grest set $k=30 \varepsilon / \sigma^{2}$ and $R_{0}=1.5 \sigma$ while the chain lengths varied between $N=5$ to $N=400$ beads for a constant monomer density $\rho=0.85 \sigma^{-3}$ [43].

\subsubsection{Mesoscopic blobs}

For the simulation of the rheological response of polymeric liquids, larger time and length scales in comparison to the polymer chain approach of Kremer and Grest are required. In order to reach these required scales, one may lump many more atoms together to represent a coarse-grained entity. For example, for a given polymer chain every 20 monomeric units can be replaced with a mesoscopic entity which is referred to a 'blob' [44-46]. This leads to the introduction of soft interactions between blobs in that they are able to pass through each other easily. To prevent the unrealistic crossing, additional restriction must be implemented for the entangled polymer system.

An algorithm incorporating a number of uncrossability constraints has been developed by Padding and Briels to explicitly detect and prevent bond crossings in mesoscopic simulations 
at high density. In this method, an 'entanglement' is considered as the crossing point between two elastic bonds. Once the crossing is detected, the entanglement point $\mathbf{X}$ at the point of the crossing is defined and a path from one blob via the entanglement to the bonded partner is formed with the pathlength $\mathbf{R}_{i, i+1}$ updated to read

$$
\mathbf{R}_{i, i+1} \rightarrow L_{i, i+1}=\left|\mathbf{R}_{i}-\mathbf{X}\right|+\left|\mathbf{X}-\mathbf{R}_{i+1}\right| .
$$

The entanglement point $\mathbf{X}$ shifts with the displacement of the blobs and will be fixed at the equilibrium position of forces. In addition the attractive potential arising due to the two crossing bonds is also updated by

$$
\phi^{a t t}\left(\mathbf{R}_{i j}\right)=c\left(\mathbf{R}_{i, i+1}\right)^{\mu} \rightarrow \phi^{a t t}\left(L_{i, i+1}\right)=c\left(L_{i, i+1}\right)^{\mu}
$$

where $c$ and $\mu$ are fitting parameters. Using this uncrossability detection technique it is possible to predict the rheology of linear polymer melts up to approximately 10 entanglement lengths and study the properties of star polymer systems [44-47].

\subsubsection{Coarse-Graining to a Single Particle}

Due to the generic nature of polymers, their chemical differences are not noticeable on large length scales i.e. polymer chain length. This naturally inspires us to develop a highly coarsegrained approach that only keeps track of the dynamics of the centre of mass of the entire polymer chain. Consequently each polymer is depicted as a single particle that interacts with other coarse-grained particles under the action of a conservative energy and dominant entanglement effects. This particle representation and interaction description is central to the algorithm referred to as Responsive Particle Dynamics (RaPiD) [48-58]. In RaPiD, transient forces are introduced to represent memory effects and to effectively describe the viscoelastic response of the polymer fluid. These memory effects are captured with two main model parameters; $n_{i j}$ which corresponds to the overlap of two interacting polymers $i$ and $j$, and $n_{0}\left(r_{i j}\right)$ which is the equilibrium number of overlaps at a distance $r_{i j}$ between two polymers. If two entangled polymers which are equilibrium are pulled apart so that $r_{i j}$ increases, $n_{i j}$ will relax over a period until it reaches $n_{0}$ for the new separation distance. During the period in which $n_{i j}$ relaxes a non-zero entanglement force develops which goes to zero once $n_{i j}$ reaches 
the new equilibrium value. This transient force can be approximately expressed as

$$
F^{\text {transient }} \propto\left(n_{i j}-n_{0}\left(r_{i j}\right)\right) \frac{d n_{0}\left(r_{i j}\right)}{d r_{i j}} .
$$

The RaPiD algorithm has proven to be an very efficient approach to study the long-time linear and non- linear rheology in linear,telechelic and star polymer systems. RaPiD has been applied for the simulation of a number of complex behaviours in polymeric fluids such as shear banding [54], colloid alignment in viscoelastic fluids [56] and non-linear flow rheology [48].

\subsection{Polymers: Basic Concepts}

Finally in this chapter a number of measures and concepts central to describing and quantifying polymer dynamics and rheology are introduced $[37,59,60]$.

\subsubsection{End-to-End Distance and Radius of Gyration}

An approximate way to describe the average size of a polymer chain is to measure the Mean Square End-to-End distance $R_{e e}$ as given by

$$
R_{e e}^{2}=\left\langle\mathbf{R}^{2}\right\rangle=\left\langle\left(\mathbf{r}_{N}-\mathbf{r}_{0}\right)^{2}\right\rangle,
$$

where $\mathbf{R}$ is defined as the vector starting from $\mathbf{r}_{0}$ to the other terminal position $\mathbf{r}_{N}$ for each configuration of the linear polymer chain. Usually this is applied to a linear chain, while for a star polymer it may be considered as the distance between the branch point to any one of the arm ends.

The extent of a polymer may also be measured by the radius of gyration $R_{g}$ which in experiments is normally measured by light scattering techniques. $R_{g}$ is calculated as from the root mean square distance of each part of the chain $\mathbf{r}_{i}$ from its center of mass position $\mathbf{r}_{G}$ using

$$
R_{g}^{2}=\frac{1}{N} \sum_{i=1}^{N}\left\langle\left(\mathbf{r}_{i}-\mathbf{r}_{G}\right)^{2}\right\rangle
$$

where $\mathbf{r}_{G}$ is calculated from

$$
\mathbf{r}_{G}=\frac{1}{N} \sum_{i=1}^{N} \mathbf{r}_{i}
$$




\subsubsection{Mean Square Displacement and Diffusivity}

The diffusion coefficient $D$ is indicative of the transition property of a polymer system. It can be calculated from the slope of the mean square displacement (MSD) of the centre of mass according to the Einstein relation,

$$
D=\lim _{t \rightarrow \infty} \frac{\left\langle(\mathbf{r}(t)-\mathbf{r}(0))^{2}\right\rangle}{6 t} .
$$

The computing time must be sufficiently long enough to accurately measure $D$ and long enough so that the chain diffuses more than its own radius of gyration.

\subsubsection{Stress autocorrelation and Viscoelasticity}

The viscoelastic character of polymer fluid can be expressed by the viscosity of the fluid. The viscosity is calculating from the integral of the time correlation function of the stress tensor for a system in equilibrium using

$$
\eta=\frac{V}{k_{B} T} \int_{0}^{\infty}\left\langle S_{\alpha \beta}(t) S_{\alpha \beta}(0)\right\rangle d t
$$

where $V$ is the volume of the system, $k_{B} T$ is the thermal energy, and $S_{\alpha \beta}$ is the $\alpha \beta$-component of the stress tensor given by

$$
S_{\alpha \beta}=-\frac{1}{V} \sum_{i}^{N}\left(r_{i, \alpha}-r_{j, \alpha}\right) F_{i j, \beta}
$$

with $r_{i, \alpha}$ being the projection of the position vector of particle $i$ on plane $\alpha$, and $F_{i j, \beta}$ the $\beta$-component of the force on $i$ due to the interaction with $j$. 


\subsection{Thesis outline}

This thesis is focused upon the simulation of architecturally complex polymer, especially in the linear and star shapes, with the polymer entities subject to varying degrees of coarse graining. We aim to study the properties of polymer melts to help towards understanding the various behaviors of polymers with differing topologies. The thesis is organized as follows.

In Chapter 2, we present a mesoscopic coarse graining model for the study of star polymer systems. The entanglement effect is accomplished by introducing the uncrossability constraint. In order to understand the structural diffusion of star polymer, the segmental diffusions are studied through the typical blob mean square displacements. Comparisons are also presented between the star polymer systems and two corresponding linear polymer chains, one with the same length as the star backbone chain and the other having the same molecular mass as the entire star. The stretched Rouse mode autocorrelation function is also investigated in this chapter.

In Chapter 3, we applied the extended coarse grained method to simulate the symmetric three-armed star polymer melts, differing in the length of the arms. The dynamical properties of melts of linear polymers are discussed to set the background. The comparison of stars with matching linear polymers are implemented to highlight the effect of polymer architecture on the bulk behavior. The scaling law of the star viscosity with molecular weight is also studied.

Asymmetric star polymers, in which the arms do not have the same length, are also discussed as well as the linear and symmetric stars in Chapter 4. In this work, two types of asymmetric stars are introduced. By varying the side asymmetric arm length, the dynamics of these polymers are compared with corresponding linear chains and symmetric stars.

In Chapter 5, we describe how we intend to calculate the RaPiD functions and parameters from the data obtained with a small scale simulation. The small scale system in this case is a three arm star polymer melt built from Kremer-Grest polymers under melt conditions. The results are demonstrated from both the thermodynamic properties and the dynamic properties.

At the end of this thesis, the results are summarized in both English and Dutch. 



\section{Coarse-grained Simulations of Moderately Entangled Star Polyethylene Melt}

In this paper, a previous coarse-grain model (J.T. Padding and W.J. Briels, J. Chem. Phys.117, 925 (2002)) to simulate melts of linear polymers has been adapted to simulate polymers with more complex hierarchies. Bond crossings between highly coarse grained soft particles are prevented by applying an entanglement algorithm. We first test our method on a virtual branch point inside a linear chain to make sure it works effectively when linking two linear arms. Next we apply our method to study the diffusive and rheological behavior of a melt of three-armed stars. We find that the diffusive behavior of the three-armed star is very close to that of a linear polymer with the same molecular weight, while its rheological properties are close to those of a linear chain with molecular mass equal to that of the longest linear sub-chain in the star.

\subsection{Introduction}

Polyethylene is among the most important commercial plastics with a variety of applications in our daily lives, such as films, extrusion coating, food packaging, etc. [3]. It can be produced in a variety of different forms based on two main structural types: high density linear polyethylene and low density branched polyethylene. It has been found that the properties of polymer melts depend sensitively on the details of the chain architecture $[4-7,11,13,14,20,30,61,62]$. Thus to have a good understanding of the properties of polymers in relation to the various possible topologies would be of great use to optimize the 
processing and synthesis techniques from both industrial and scientific perspectives.

The dynamical and rheological properties of linear polymer systems are described quite well by the Rouse model for short chains [34] and the reptation model for entangled chains [63]. The experimentally observed scaling laws for the melt self-diffusion coefficient and viscosity as a function of molecular weight are explained successfully by these models $[31,64$, 65]. Besides this, computational methods have been developed to test theoretical predictions and reveal new phenomena along with experiments in this field.

Among the computer simulations, fully atomistic molecular simulations are usually carried out to acquire accurate fundamental information on polymer structures and dynamics in relation to their chemical details $[8,25,32,33,66]$. However, with current computer capabilities these goals become unaccomplishable with highly entangled systems, where long relaxation times and large simulation boxes have to be dealt with. In cases when less detailed information is needed to understand the basic physical phenomena of interest, coarse-graining techniques offer an alternative and efficient way to reach long time and length scales. For example, when studying the flow properties of linear polymers, one is mainly interested in some generic properties of entire chains [40-42,44,45,67].

Despite the advantages of coarse grained simulations, one should be aware of the fact that the coarse grain potential will become increasingly softer on lumping more and more atoms together, and consequently bonds will finally be capable of crossing each other. Such illegal bond crossings may be prevented by a number of uncrossability constraints. One has been developed by Padding and Briels in a method, called TWENTANGLEMENT, based on introducing an 'entanglement' at the touching location of two elastic bonds [44]. During the remaining part of the run, the entanglement points are updated with every time step by minimizing the elastic energy in the two entangled bonds, until they disentangle again. This model has been shown to be able to describe the effects of entanglements and to reproduce effectively experimental data of moderately entangled linear polymer melts [45].

In this work, we extend the program to be applicable to architecturally complex systems. Roughly speaking, we have updated the entanglement algorithm to prevent crossings between all branches of all polymers. Star polymers with a single branch point architecture present the simplest example of a branched polymer, and have attracted scientific interests for a long time $[9,10,15-18]$. We therefore first apply our model on star polymers. 
The organization of this paper is as follows: in Section 2.2 we describe the coarse-grained simulation model and introduce the uncrossability constraint. The topology specification is also discussed in this part. In Section 2.3 the properties of star polymer are presented and we make the comparisons between 3-arm star and linear chains. Section 2.4 contains the conclusions of this work.

\subsection{Model overview and Simulated systems}

\subsubsection{Coarse-graining method and Brownian dynamics}

Considering that the chemical details are unimportant when studying the dynamical properties of polymers at large time and length scales, we group a large number, $\lambda=20$, of monomers together into one coarse-grained bead (also referred to as blob). Accordingly a coarse-grained chain can be described by several consecutive blobs. For example, a linear polyethylene chain $\mathrm{C}_{140} \mathrm{H}_{282}$ in this work is represented by seven blobs and named $\mathrm{B}_{7}$.

For each blob, the center of mass position $\mathbf{R}$ is the average of $\lambda$ monomer locations $\mathbf{r}_{i}$, which are weighted by their mass $m_{i}$,

$$
\mathbf{R}=\frac{1}{M} \sum_{i=1}^{\lambda} m_{i} \mathbf{r}_{i}
$$

where $M$ is the blob's total mass. Blobs are simply treated as Brownian particles and their center of mass positions are evolving through a time dependent first-order Langevin equation, which includes displacements due to forces and random displacements $\delta \mathbf{R}_{i}$

$$
d \mathbf{R}_{i}=-\frac{1}{\xi} \nabla_{i} \Phi d t+\delta \mathbf{R}_{i}
$$

where $\Phi$ is the potential energy and $\xi$ the blob friction coefficient. We assume that the friction coefficients are independent of the blob configurations, and are related to the stochastic displacements through the fluctuation-dissipation theorem:

$$
\left\langle\delta R_{i \alpha} \cdot \delta R_{j \beta}\right\rangle=\frac{2 k_{B} T}{\xi} d t \delta_{i j} \delta_{\alpha \beta},
$$

where Greek subscripts refer to coordinate directions, $k_{B}$ is Boltzmann's constant, and $T$ is the temperature of the system. 
Forces between blobs are derived from the potential of mean force, which is related to the $n$-blob probability distribution function $P_{n}$ according to

$$
\Phi\left(\mathbf{R}^{n}\right)=-k_{B} T \ln P_{n}\left(\mathbf{R}^{n}\right)
$$

where $P_{n}$ may be obtained from an appropriate atomistic simulation.

We assume that the total potential of mean force can be approximately expressed by three independent contributions: a sum over all non-bonded pairs, one over bonded blobs, and one over bond angles

$$
\Phi\left(\mathbf{R}^{n}\right)=\sum_{i<j} \varphi^{n b}\left(R_{i, j}\right)+\sum_{i} \varphi^{b}\left(R_{i, i+1}\right)+\sum_{i} \varphi^{\theta}\left(\theta_{i}\right)
$$

The inter- and intra-molecular interactions together with potential functions are shown in Table 2.1. A Gaussian pair potential is used to describe repulsive forces between non-bonded $\varphi^{n b}$ and bonded $\varphi^{b_{\text {rep }}}$ blobs. The attractive part $\varphi^{b_{\text {att }}}$ for bonded interaction is described by a single power law. And the bending potential $\varphi^{\theta}$ is a cosine function of the angle $\theta$ between two consecutive bonds.

\begin{tabular}{ll} 
interaction type & potential expression \\
\hline non-bonded & $\varphi^{n b}(R)=c_{0} e^{-\left(R / b_{0}\right)^{2}}$ \\
bonded repulsive & $\varphi^{b_{\text {rep }}}(R)=c_{1} e^{-\left(R / b_{1}\right)^{2}}+c_{2} e^{-\left(R / b_{2}\right)^{2}}$ \\
bonded attractive & $\varphi^{b_{\text {att }}}(R)=c_{3}(R)^{\mu}$ \\
bending potential & $\varphi^{\theta}(\theta)=c_{4}(1+\cos \theta)^{v}$
\end{tabular}

Table 2.1: Interactions between non-bonded and bonded blobs.

The blob interactions are of course more complicated than assumed here. However, it is known that entanglement effects play a dominant role in the rheology of polymeric systems. This allows us to neglect to a large extent all details of the interactions in our simulation work. Similar assumptions are made in several other rheological simulation methods like in the works of the Naples group [68-70], and in the work of Schieber [71,72] and Likhtman [7375]. All these entanglement-based methods have the drawback that the diagonal elements of the stress tensor are not necessarily correct and that thermodynamic properties in general 
are not described at all. A coarse grain model that does describe both thermodynamics and rheology correctly was introduced by our group some time ago and now goes with the name RaPiD $[50,51,55,56,58]$. This model, however, does not explicitly include the architectural issues that we want to address in this paper.

\subsubsection{Uncrossability constraint}

As mentioned in the previous section, every 20 monomer units are lumped together to form a highly coarse grained particle. Due to the high coarse-graining level, the interaction potential of blobs becomes very soft and bonds can cross each other. In order to prevent the occurrence of this kind of unrealistic phenomenon, we implement an uncrossability constraint algorithm (see below), which introduces entanglements when imminent bond crossings are detected. The number of monomers mapped on one blob on the one hand satisfies the need for a large integration time step, and on the other hand assures that the size of the blobs is smaller than one reptation tube diameter. This allows for the possibility of reptation to emerge from the simulation. A coarse graining to blobs larger than the tube diameter will smear out confinement effects, resulting from the potential interactions and the imposed uncrossability constraints, and show reptation at an incorrect length scale, unless reptational moves are introduced by hand at the level of the blobs.

The uncrossability constraint is based on the consideration that bonds between blobs have elastic properties. By means of a geometric check, as soon as two bonds are detected to touch each other, an entanglement point $\mathbf{X}$ will be created at the crossing site. Concomitantly, the attractive potential contributed by the two crossing bonds is changed by replacing the two blob distances $R_{i, i+1}$ by pathlengths $L_{i, i+1}$ :

$$
\varphi^{a t t}\left(L_{i, i+1}\right)=c_{3}\left(L_{i, i+1}\right)^{\mu} .
$$

The pathlength $L_{i, i+1}$ is the contourlength of the path that starts at blob $i$ and goes via a number of entanglement points $\mathbf{X}_{1}, \mathbf{X}_{2}, \ldots, \mathbf{X}_{p}$, to the next blob $i+1$.

$$
\begin{aligned}
L_{i, i+1} & =\left|\mathbf{R}_{i}-\mathbf{X}_{1}\right|+\left|\mathbf{X}_{1}-\mathbf{X}_{2}\right|+\ldots \\
& +\left|\mathbf{X}_{p-1}-\mathbf{X}_{p}\right|+\left|\mathbf{X}_{p}-\mathbf{R}_{i+1}\right| .
\end{aligned}
$$

With every time step the 'entanglements' so created are updated such as to minimise the total attractive bond energy, and thereby forbid chain crossing. We stress that the number 


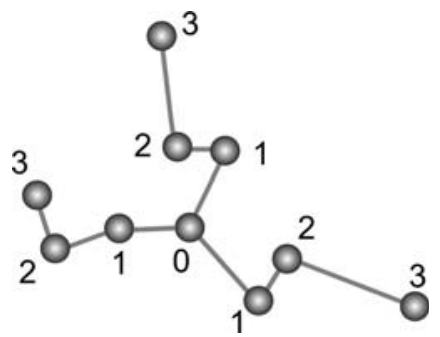

Figure 2.1: Schematic representation of $B\left(B_{3}\right)_{2}$ with a branch point and three arms.

of entanglements so defined is not the same as the entanglement number $\mathbf{Z}$ as it is used in reptation theory. It is rather the long-lasting entanglements which define the entanglement number. We refer to the original paper by Padding and Briels for a distribution of entanglement lifetimes for the linear chains.

\subsubsection{Topology specification and simulation details}

We conduct our simulations with linear and star polyethylene melts at a temperature of $450 \mathrm{~K}$. The numberings of a 3-arm star polymer are shown in Fig. 2.1. The name of a star polymer is defined by putting information of the branch point into the first bracket, and after that the length and number of star arms. For example, $\mathrm{B}\left(\mathrm{B}_{3}\right)_{3}$ consists of one branch point to which three arms are attached, each consisting of three blobs. Linear chains of $n$ blobs in this scheme are simply called $\mathrm{B}_{n}$. Notice that the properties of, for example, $\mathrm{B}_{7}$ and $\mathrm{B}\left(\mathrm{B}_{3}\right)_{2}$ should be the same.

The main complication with the simulation of star polymers is due to the presence of a branch point. In this paper we build stars by linking together linear chains to one central branch point. Any two linear chains connected at a branch point should then communicate through appropriate potential forces not present before the connection. Moreover entanglements should be dealt with correctly.

In view of the algorithmic complications, we first implemented a simple test to ensure that a branch point works properly when linking together two linear chains. The general idea is displayed in Fig. 2.2, where two $\mathrm{B}_{4}$ chains are connected at a branch point by overlapping 

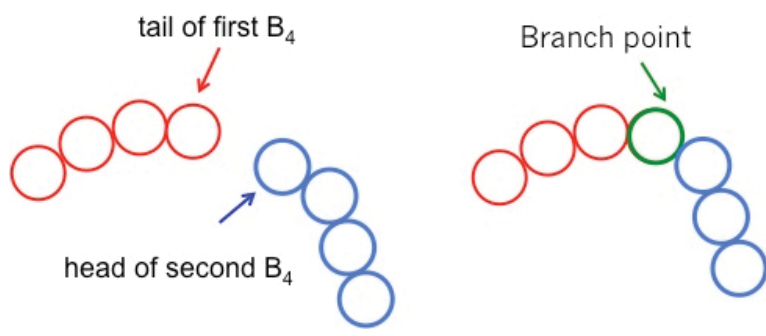

Figure 2.2: Sketch of $B\left(B_{3}\right)_{2}$ on the right with a branch point made by joining two $B_{4}$ chains on the left.

the tail and head blob of the first and second $\mathrm{B}_{4}$ chain. Consequently a new chain, called $\mathrm{B}\left(\mathrm{B}_{3}\right)_{2}$ with a branch point $\mathrm{B}$ and two side arms $\left(\mathrm{B}_{3}\right)_{2}$ is created. We assume that the branch point has a mass and friction equal to that of a normal blob. Provided that the two originally separated $\mathrm{B}_{4}$ chains now communicate their entanglements through the shared branch point in a correct way, the newly formed $\mathrm{B}\left(\mathrm{B}_{3}\right)_{2}$ should be equivalent with a single $\mathrm{B}_{7}$ according to the effective blob number. Therefore, entanglements which slip over the branch-end of one chain should not be eliminated, as they would be at a regular end of a chain, but be recognized as entanglements at the partner chain at the other side of the branch point. Only if this is done correctly will the correct results be obtained for the longer chain. The procedure followed by us at branch points of functionality three will be described below.

Blob and center of mass mean square displacements of $\mathrm{B}\left(\mathrm{B}_{3}\right)_{2}$ and $\mathrm{B}_{7}$ chains are shown in Fig. 2.3. The simulation parameters are given in Table 2.2. From this plot, it is seen that the new linear chain with a branch point inside, diffuses in exactly the same way as $\mathrm{B}_{7}$. We also make a comparisons of the rheological properties of both systems expressed by their zero shear stress relaxation moduli. In Fig. 2.4, the shear relaxation modulus of $\mathrm{B}\left(\mathrm{B}_{3}\right)_{2}$ very well follows that of $\mathrm{B}_{7}$. We conclude that the branch point works perfectly well in terms of chain dynamics.

Now shifting our attention to branch points in multi-armed stars, we need to take care that the angle between two bonds connected through a branch point should be treated differently than the one in a linear chain. The bonds inside a linear chain form angles taking any value between zero and $\pi$. With three-armed stars we expect that the arms at the branched point will 


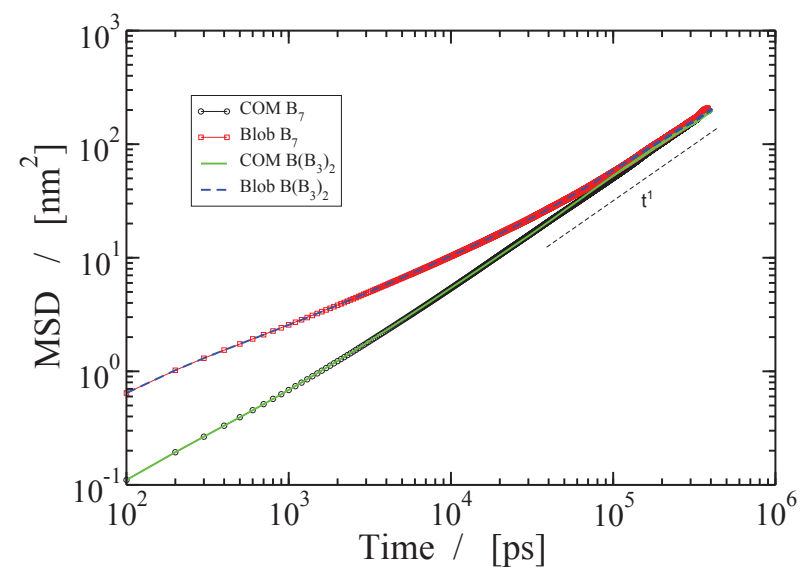

Figure 2.3: Diffusion of $B_{7}$ and $B\left(B_{3}\right)_{2}$. For both center of mass and blobs, these two chains diffuse in the same way.

on average be co-planar and make angles of $2 / 3 \pi$. In order to encourage our coarse-grained system to take this configuration, we have changed the bending potential at the branch point to

$$
\varphi_{b p}^{\theta}(\theta)=c_{5}\left(\cos \theta-\cos \frac{2}{3} \pi\right)^{2} .
$$

In Fig. 2.5 we have plotted the observed probability distributions (pdf), both for the bond angles along the arms (panel a) and for those at the branch points (panel b), with parameters given in Table 2.2. Notice that the pdf for the branch points has its maximum at a value of $\cos \theta$ slightly higher than -0.5 , i.e. at angles slightly smaller than $2 / 3 \pi$. The reason for this is that with non co-planer bonds the total sum of the bond angles is less than $2 \pi$.

We finally must decide what happens to entanglement that slip over the branch point. Consider an entanglement that 'lives' on arm number one and slips over the branch point, as illustrated in Fig. 2.6. After this move the entanglement may find itself in one of four situations, it may have been annihilated, it may be entangled either with arm number two or number three, or it may be entangled with both arms number two and number three. We have implemented the following algorithm. After the slip, we randomly choose either arm number 


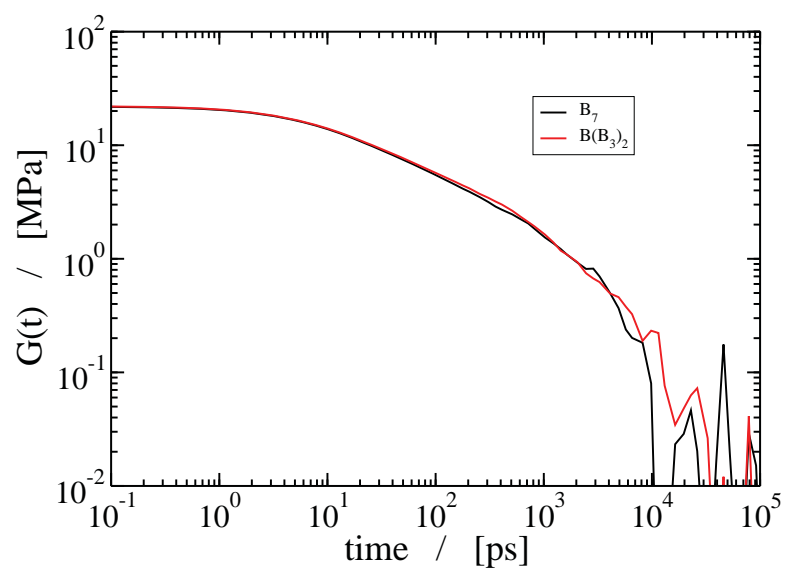

Figure 2.4: Stress autocorrelation function of $B_{7}$ and $B\left(B_{3}\right)_{2}$, they are following the same relaxation behavior.

two or arm number three. For the chosen arm we perform the usual entanglement checks after a slip over a blob. If an entanglement occurs with this arm, we stick to this entanglement ignoring other possibilities. If no entanglement occurs with this arm, we perform the entanglement checks for the remaining arm. If an entanglement does occur with this arm, we stick to this one and continue the run. In case also with this arm no entanglement survives, we annihilate the entanglement and continue the run. This algorithm will give the correct result in case the entanglement is finally annihilated. In case exactly one entanglement should remain, the algorithm will find the correct arm. In case two entanglements should occur, the algorithm only creates one on a randomly chosen arm. A large number of geometrical checks would be required to check for double entanglements. All results with the present algorithm are therefore valid under the assumption that double entanglements are relatively rare.

To generate an equilibrated box, we randomly place a number of polymers into the simulation box, keeping the mass density equal to its melt value of $\rho=0.761 \mathrm{~g} / \mathrm{cm}^{3}$. Unentangled simulations are performed first to allow a thorough equilibration. Then the uncrossability constraints are switched on to add the entanglement effect. The parameters for the potential of mean forces of this model are shown in Table 2.2. They apply when $\lambda=20$, and are fit to the distribution functions obtained in atomistic simulations [44]. 


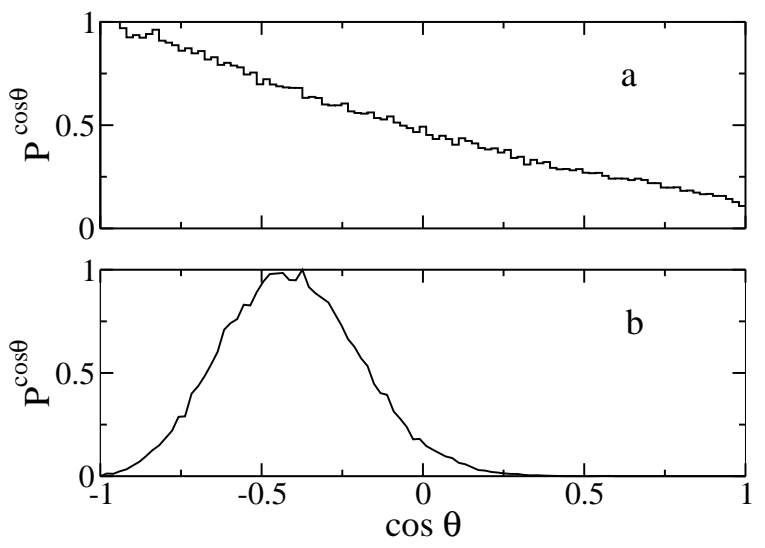

Figure 2.5: Probability distributions of bending angles in arbitrary unit. Panel a shows the bond angles along the arms, and curve in panel $b$ for those at the branch points.

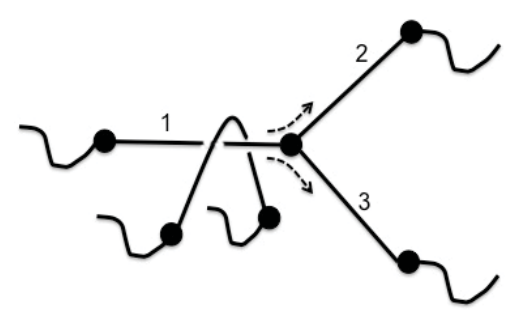

Figure 2.6: Diagram of an entanglement slipping past a branch point.

\subsection{Results}

In this section we discuss typical blob time dependent mean square displacements. To this end we number the blobs along the arms of each star from zero to three. With each star and each arm, blob number zero is the branch point and blob number three is the end point, shown in Fig. 2.1. Blob number one is the blob which is topologically closest to the branch point and blob number two is closest to the end point. The mean square displacement for blobs of type $i$ is then

$$
\mathbf{M S D}_{i}=\left\langle\left[\mathbf{R}_{i}(t)-\mathbf{R}_{i}(0)\right]^{2}\right\rangle,
$$




\begin{tabular}{llll} 
types & parameter & value & unit \\
\hline non-bonded & $\mathrm{c}_{0}$ & 5.56 & $\mathrm{~kJ} \mathrm{~mol}^{-1}$ \\
& $\mathrm{~b}_{0}$ & 0.49 & $\mathrm{~nm}$ \\
\hline \multirow{2}{*}{ bonded repulsive } & $\mathrm{c}_{1}$ & 10.46 & $\mathrm{~kJ} \mathrm{~mol}^{-1}$ \\
& $\mathrm{c}_{2}$ & 0.26 & $\mathrm{~nm}$ \\
& $\mathrm{~b}_{2}$ & 0.67 & $\mathrm{~nJ} \mathrm{~mol}$ \\
\hline \multirow{2}{*}{ bonded attractive } & $\mathrm{c}_{3}$ & 6.46 & $10^{-3} \mathrm{~kJ} \mathrm{~mol}^{-1} \mathrm{~nm}^{-\mu}$ \\
& $\mu$ & 9.73 & \\
\hline \multirow{2}{*}{ angular } & $\mathrm{c}_{4}$ & 3.01 & $\mathrm{~kJ} \mathrm{~mol}^{-1}$ \\
& $\mathrm{c}_{5}$ & 24.08 & $\mathrm{~kJ} \mathrm{~mol}^{-1}$ \\
& $v$ & 1.2 &
\end{tabular}

Table 2.2: Parameters for potential of mean force.

here the pointy brackets denote the usual average over initial times and an average over all blobs of type i, i.e. over all stars and all arms. Besides these we will also discuss the averaged blob mean square displacement, $\mathbf{M S D}_{b l}=\frac{3}{10} \sum_{i=1}^{3} \mathbf{M S D}_{i}+\frac{1}{10} \mathbf{M S D}_{0}$ and $\mathbf{M S D} \mathbf{D}_{c m}=$ $\left\langle\left[\mathbf{R}^{c m}(t)-\mathbf{R}^{c m}(0)\right]^{2}\right\rangle$, with the $\mathbf{R}^{c m}$ being the center of mass of the star.

Furthermore we will discuss the shear relaxation modulus $G(t)$, i.e. the linear stress response to a step strain. It can be measured from equilibrium fluctuations of the stress tensor as follows,

$$
G(t)=\frac{V}{k_{B} T}\left\langle\sigma_{\alpha \beta}(t) \sigma_{\alpha \beta}(0)\right\rangle, \alpha \neq \beta
$$

with $V$ the total volume of the simulation box, $\alpha, \beta \in\{x, y, z\}$ are different coordinate directions, and $\sigma_{\alpha \beta}$ is the microscopic stress tensor:

$$
\sigma_{\alpha \beta}=-\frac{1}{V} \sum_{i, j}\left(r_{i, \beta}-r_{j, \beta}\right) F_{i j, \alpha}
$$

where $\mathbf{r}$ is the blob position vector, and $F_{i j, \alpha}$ is the $\alpha$ component of the force on blob $i$ exerted 


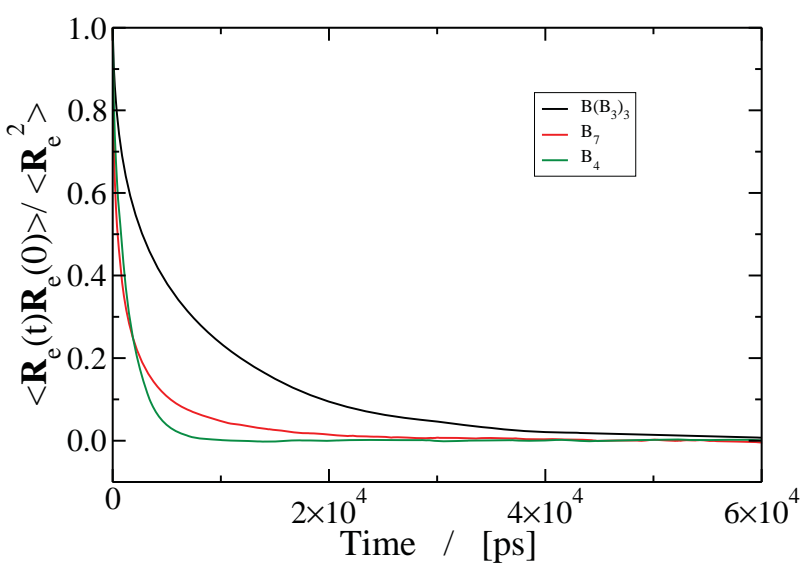

Figure 2.7: Time autocorrelation function of the unit vector directed from the branch point to the end of three arms for $B\left(B_{3}\right)_{3}$, and end-to-end vector for linear $B_{4}$ and linear $B_{7}$.

by blob $j$. From $G(t)$ we obtain the storage $G^{\prime}(\omega)$ and loss modulus $G^{\prime \prime}(\omega)$ in the usual way,

$$
\begin{aligned}
G^{\prime}(\omega) & =\omega \int_{0}^{\infty} \sin (\omega t) G(t) d t, \\
G^{\prime \prime}(\omega) & =\omega \int_{0}^{\infty} \cos (\omega t) G(t) d t .
\end{aligned}
$$

\subsubsection{Dynamics and rheological properties of star polymer}

In order to confirm the complete relaxation of our stars at the longest chain length possible, we have calculated time dependent autocorrelation functions (ACF) for the unit vectors directed from the branch point to the end-points of the star arms. The results are shown in Fig. 2.7 below, together with those for the end-end vectors of linear chains. It is clear from this picture that, even when the decay of the branch-end vectors for our star is very slow, many decay-times fit within the total runtime $10^{6} \mathrm{ps}$. We are assured therefore that our system is well equilibrated. Similar conclusions may be drawn from the mean-square displacements discussed next.

In Fig. 2.8 we display the blob and center of mass mean square displacements both when entanglements are accounted for (black curves) and when entanglement have been ignored (red curves). As is clear from the plots, entanglements substantially slow down the almost 


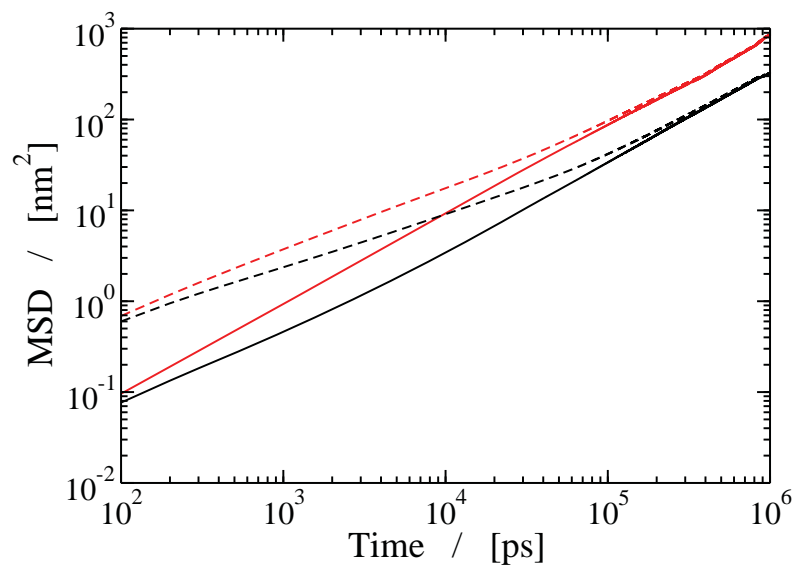

Figure 2.8: Blob mean square displacement (dashed) and center of mass mean square displacement (solid) of $B\left(B_{3}\right)_{3}$ with (black) and without (red) entanglement.

Rouse-like mean square displacements obtained for non-entangling polymers.

In order to better understand the structural diffusion of entangled stars, we have measured typical blob mean square displacements and plotted them in Fig. 2.9. As a result of the symmetrical arrangement of the various blobs in $\mathrm{B}\left(\mathrm{B}_{3}\right)_{3}$, blobs on equivalent positions along the three arms diffuse according to identical curves. The curves in Fig. 2.9 can therefore be separated into four groups, from bottom to top corresponding to segmental motions of the innermost branch point to the outermost free ends of each of the arms of the star. At early times, because of topological constraints originating along three different arms, the central branch point moves very slowly, in fact almost immediately as slow as in its final diffusive regime (solid black). The blobs next to the branch point move substantially faster than the branch point and become diffusive at a much later time. This trend continues with blobs being situated increasingly further outwards along the arms, with the arm end being the fastest of all, becoming diffusive only at a very late time.

In order to analyse the dynamics of the various blobs even further, we have calculated Rouse modes and their time correlation functions. In the Appendix 2.4 we shortly review the application of the Rouse model to non-linear polymers. From a mathematical point of view the analysis in the Appendix amounts to a transformation from blob-coordinates $\mathbf{R}_{k}$ to Rouse 


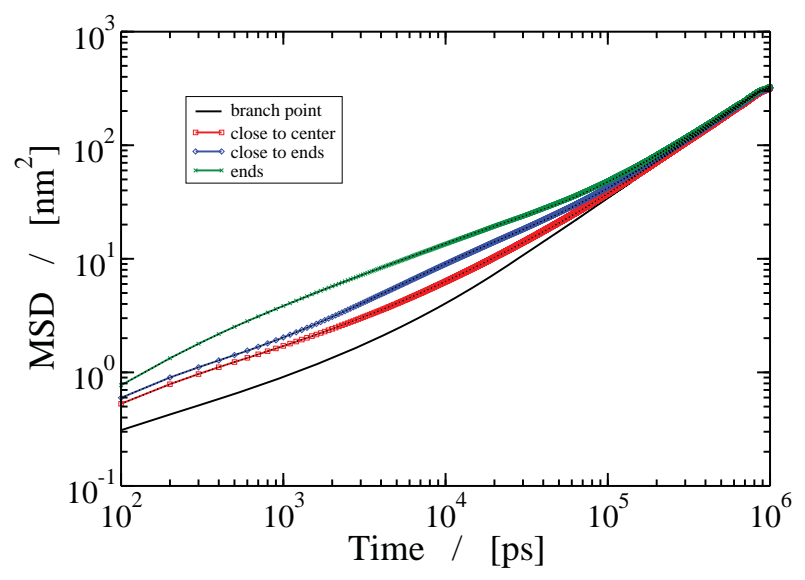

Figure 2.9: Mean square displacement of each blob in $B\left(B_{3}\right)_{3}$ with entanglement. From the bottom to top, the curves are corresponding to the from innermost branch point to outermost free ends along each star arms.

coordinates $\mathbf{X}_{k}$ which are chosen such that the coupled motion of the blobs in the Rouse model become decoupled. The time correlation functions of the Rouse coordinates, Rouse mode correlation functions for short, within the Rouse model decay exponentially with relaxation times $\tau_{k}$. In case the Rouse model does not apply, the Rouse coordinates still provide interesting coordinates to describe the motion of the blobs, in particular to reveal deviations from Rouse behavior. Due to interactions between non-bonded blobs and the uncrossability constrains, the Rouse mode time correlation functions will not follow the exponential decay, and may usually be described reasonably well by the so called stretched exponentials according to:

$$
C_{k}(t)=\left\langle\left(\mathbf{X}_{k}(t) \cdot \mathbf{X}_{k}(0)\right)\right\rangle /\left\langle\mathbf{X}_{k}^{2}\right\rangle=\exp \left[-\left(t / \tau_{k}^{*}\right)^{\beta_{k}}\right]
$$

the relaxation time $\tau_{k}^{*}$ and stretching parameter $\beta_{k}$ are obtained for each Rouse mode number $k$ by fitting Eq. (2.13) to the simulated normalized Rouse mode autocorrelation $C_{k}(t)$. Notice that $\beta_{k}$ is equal to one in case the Rouse model applies.

In Fig. 2.10 we have plotted $C_{k}(t)$ both for simulations with and without taking entanglements into account. For all modes the unentangled Rouse mode correlations are perfectly 


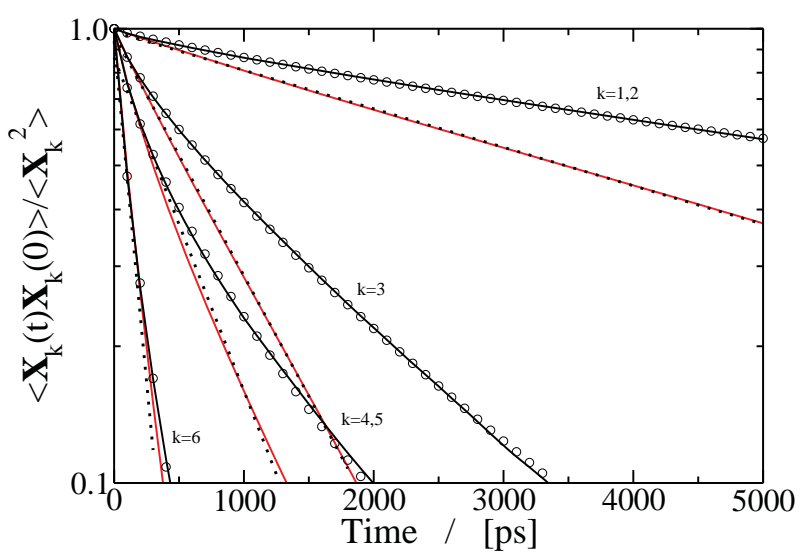

Figure 2.10: Mode relaxations in $B\left(B_{3}\right)_{3}$, scaled as $\ln \left[C_{k}(t)\right]$ on a linear scale. The solid black and red curves are the modes corresponding to the system with and without entanglements. The circles and dotted lines are the respective fits for each mode number $k$.

well described by simple exponentially decaying functions, indicating that the soft potential Eq. (2.5) does not lead to non-Rouse behavior. Moreover, at very short times, the Rouse mode correlation functions of the entangled and non-entangled simulations are tangential to each other. Quickly, however, entanglements severely slow down the Rouse mode correlations such that they cannot be described anymore by simple exponentials, although the stretched exponentials of Eq. (2.13) still do a very good job.

According to Eq. (A.12), the blob positions can be calculated from the Rouse mode coordinates. Assuming that in the real system Rouse modes are still not correlated, blob mean square displacements may be calculated according to

$$
\left\langle\left[\mathbf{R}_{n}(t)-\mathbf{R}_{n}(0)\right]^{2}\right\rangle=6 D_{R} t+2 \sum_{k=1}^{N-1} S_{n k}^{2}\left\langle\mathbf{X}_{k} \cdot \mathbf{X}_{k}\right\rangle\left(1-C_{k}(t)\right) .
$$

In Fig. 2.11, the solid curves are the blob displacements calculated from the simulation. The dotted lines are calculated according to Eq. (??) using the simulated diffusion coefficient $D_{R}$ and the exponential representation of the Rouse mode correlation functions (upper group of curves, left vertical axis) or the stretched exponential representation of the Rouse mode correlation functions (lower group of curves, right vertical axis). It is clearly seen that the 


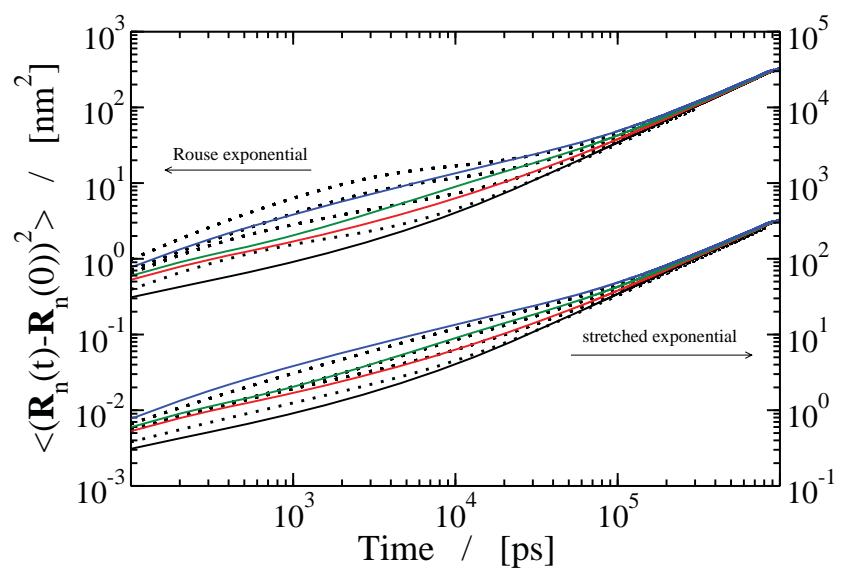

Figure 2.11: Blob displacements of $B\left(B_{3}\right)_{3}$. The dotted lines are numerical calculations from the modes: the upper groups are using the Rouse exponential fit, and the lower groups are the ones from stretched exponential fit. The solid lines are simulation results for comparisons.

Rouse model representation, i.e. the exponential representation of the Rouse mode correlation functions, leads to blob motions much faster than the actual motions, while the stretched exponential representation gives almost correct results. The remaining deviations in the latter case may be caused by correlations between different Rouse modes, or by small deviations of the Rouse mode correlation functions from their stretched exponential fits.

We now turn our attention to the viscoelastic properties of our star polymer $\mathrm{B}\left(\mathrm{B}_{3}\right)_{3}$ melt. In Fig. 2.12, the storage and loss moduli, $G^{\prime}(\omega)$ and $G^{\prime \prime}(\omega)$, are plotted as a function of frequency. Both curves are calculated as the appropriate Fourier transform of the stress relaxation modulus $G(t)$, shown in the inset. In the low frequency region $G^{\prime}(\omega)$ and $G^{\prime \prime}(\omega)$ are proportional to $\omega$ and $\omega^{2}$ respectively. The crossing of both curves occurs when $\omega$ approximately equals $10^{-3} / \mathrm{ps}$, which means that the longest relaxation time of $\mathrm{B}\left(\mathrm{B}_{3}\right)_{3}$ is roughly equal $t=\omega^{-1}=10^{3} \mathrm{ps}$. At frequencies beyond the crossing point, $G^{\prime}(\omega)$ becomes larger than $G^{\prime \prime}(\omega)$, and both curves continue to increase up to the highest frequency that can be investigated with the present coarse grain model. The continuous increase of the loss modulus after crossing the storage modulus is in agreement with experimental [61] and theoretical [13,62] predictions for small stars. Since our star is rather short, the slope of the loss modulus is still 


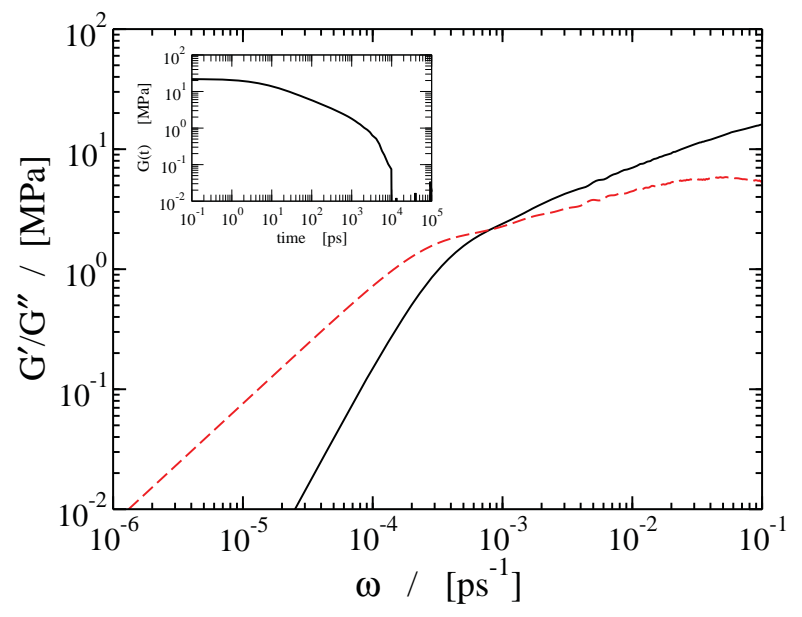

Figure 2.12: The storage and loss moduli, $G^{\prime}(\omega)$ and $G^{\prime \prime}(\omega)$, of $B\left(B_{3}\right)_{3}$ system, transformed from stress relaxation curve $G(t)$ on top left.

rather large. Only for very large stars will the slope practically become zero; this holds true both for Rouse stars as well as for entangled stars.

\subsubsection{Comparisons between star and linear polymers}

Segmental motions in general reveal (part of) the topological constraints in complex polymers. In order to better understand the relation between topological constraints and architectural complexity, we compare the conformational and dynamical properties of $\mathrm{B}\left(\mathrm{B}_{3}\right)_{3}$ and those of related linear chains. We selected three linear chains for comparison: $\mathrm{B}_{4}$, which corresponds to the length of one arm, $\mathrm{B}_{7}$, which corresponds to the longest linear backbone in $\mathrm{B}\left(\mathrm{B}_{3}\right)_{3}$ between two ends, and $\mathrm{B}_{10}$, corresponding to a linear chain having the same number of blobs or molecular weight as the target star.

The radius of gyration $R_{g}$ can be calculated directly from the simulated configurations, or assuming Rouse-like behavior and using

$$
R_{g}=\sqrt{\frac{1}{N} \sum_{k=1}^{N-1}\left\langle\mathbf{X}_{k} \cdot \mathbf{X}_{k}\right\rangle}=b \sqrt{\frac{1}{N} \sum_{k=1}^{N-1} \frac{1}{\lambda_{k}}} .
$$


where $\mathrm{b}$ is the Kuhn length and $\lambda_{k} \mathrm{~s}$ are the eigenvalues of the connectivity matrix $\mathrm{T}$ (see Appendix). The value of $R_{g}^{2}$ for $\mathrm{B}\left(\mathrm{B}_{3}\right)_{3}$ is $4.065 \mathrm{~nm}^{2}$, and the ratio of $R_{g}^{2}$ of $\mathrm{B}\left(\mathrm{B}_{3}\right)_{3}$ to that of $\mathrm{B}_{10}$ turns out to be 0.86 , which is a bit larger than the theoretical or experimental value $0.8[76,77]$ obtained with very large molecular weights.

It is interesting to compare the branch-end vector autocorrelation function of the star arms with the end-end vector autocorrelation functions of various linear chains as shown in Fig. 2.7. As is to be expected, the star arms relax much slower than the corresponding linear chain $\mathrm{B}_{4}$. The slowing down effect of connecting three arms together, is actually so strong that the arm relaxation is even much slower than the end-end vector relaxation of the longest chain present in the star, i.e. $\mathrm{B}_{7}$.

We next compare star and linear chains by their center of mass mean square displacements and zero shear relaxation moduli. Fig. 2.13 reveals that the MSD curve of $\mathrm{B}\left(\mathrm{B}_{3}\right)_{3}$ (solid line) strongly overlaps with the one of $\mathrm{B}_{10}$ (circle symbol) and is substantially slower than that of $\mathrm{B}_{7}$. Notice that our linear stars are too small to show the various regimes in the (blob)mean square displacements that are so characteristic for reptational motion. In fact Our longest chain is a bit shorter than two entanglement lengths. We refer to the original papers of Padding and Briels for further information on this issue [45]. Although somewhat boldly, we conclude/surmise that relatively small stars diffuse similar to linear polymers of equal mass; on the basis of the present results, nothing can be said about the diffusive behavior of much larger stars.

In contrast, Fig. 2.14 shows that the stress relaxation of $\mathrm{B}\left(\mathrm{B}_{3}\right)_{3}$ is much faster than that of $\mathrm{B}_{10}$, very akin to that of $\mathrm{B}_{7}$. Intuitively one may say that stress fluctuations are roughly proportional to the largest distance between two points of applied forces. These are very similar in $\mathrm{B}\left(\mathrm{B}_{3}\right)_{3}$ and $\mathrm{B}_{7}$, and substantially shorter than in $\mathrm{B}_{10}$. By integrating $\mathrm{G}(\mathrm{t})$ we have calculated zero shear rate viscosities, finding $\eta_{0}\left(\mathrm{~B}_{7}\right)=9.22 \times 10^{-3} \mathrm{~Pa} \mathrm{~s}, \eta_{0}\left(\mathrm{~B}_{10}\right)=32.3 \times 10^{-3} \mathrm{~Pa} \mathrm{~s}$, and $\eta_{0}\left(\mathrm{~B}\left(\mathrm{~B}_{3}\right)_{3}\right)=9.19 \times 10^{-3} \mathrm{~Pa}$ s. For the two linear polymers, these results are in agreement with those in ref. [45], and in good agreement with experiments. We could not compare the star polymer to experimental results, because synthesized stars typically have much larger arms. 


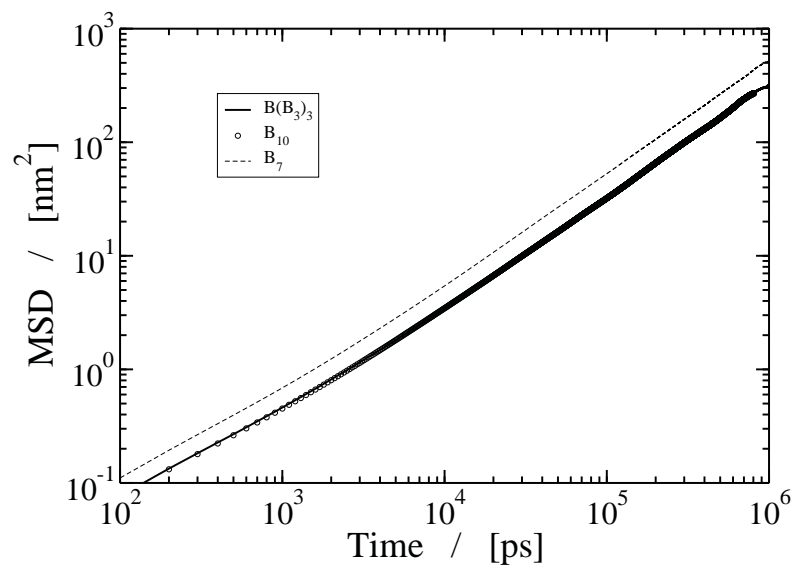

Figure 2.13: Comparisons of center of mass mean square displacement in $B\left(B_{3}\right)_{3}, B_{10}$ and $B_{7}$.

\subsection{Conclusions}

In this work, the TWENTANGLEMENT code to perform coarse-grain simulations of linear polymers has been made applicable to polymers of more complex architectures. In order to deal with complex architectures we have included the possibility to connect several polymers at one branch point (of course one complex polymer may contain more than one branch point). We have tested our code by connecting two linear chains into a longer linear chain. Next we applied our method to build a star polymer $\mathrm{B}\left(\mathrm{B}_{3}\right)_{3}$ and investigated its dynamical and rheological properties. The results were compared with those of linear melts with molecular weight equal to that of the entire molecule or that of the main backbone chain consisting of two arms and the branch point of our three-arm star.

From our simulations, we found that the shape of the polymer has an important effect on the properties of the melt. We observed different dynamics for different star polymer segments. Rouse mode correlation functions could be well described by stretched exponentials. Assuming uncorrelated Rouse modes we recovered the blob mean square displacements almost completely, meaning that different Rouse modes are only slightly correlated. The comparison with linear chains revealed that (relatively short) star polymers diffuse as linear 


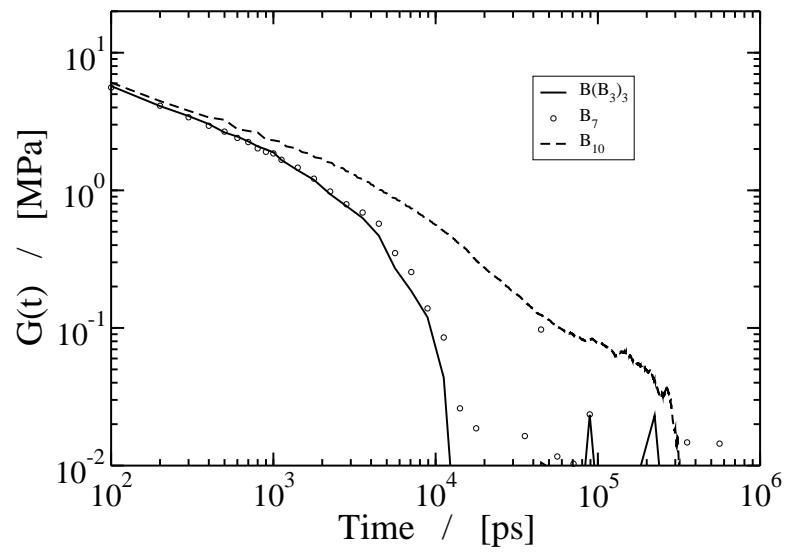

Figure 2.14: Comparisons of zero shear relaxation in $B\left(B_{3}\right)_{3}, B_{10}$ and $B_{7}$.

chains of equal mass whereas they display rheological behavior very similar to that of the linear polymer with a molecular mass equal to that of the longest linear chain available in the star.

Obviously each arm of our $\mathrm{B}\left(\mathrm{B}_{3}\right)_{3}$ represents a rather short polymer, approximately a bit less than one entanglement length, but still long enough to capture some characteristics of a star polymer melt that is different from a linear polymer melt. Future work will focus on simulations of stars having longer entangled arms and different numbers of arms to understand the chain length and branching degree effect. Efforts are also in progress aiming at simulating branched polymers of other shapes.

\section{APPENDIX: ROUSE MODEL OF NONLINEAR POLYMERS}

In this Appendix we quickly review the salient features of the Rouse model as applied to non-linear polymers. We collect all position vectors $\mathbf{R}_{n}$ in one long vector $R$. To be clear, every entry of $R$ is a vector, not one of its Cartesian coordinates. The total number of entries in $R$ is therefore equal to the number of blobs $N$ in each polymer. The equation of motion then reads

$$
\frac{d R}{d t}=-w T R+F,
$$


here $T$ is a symmetric matrix reflecting the topology of the polymer and $F$ is a vector of random displacements, for which

$$
\begin{aligned}
& \langle F(t)\rangle \quad=0, \\
& \left\langle F(t) \cdot F(0)^{T}\right\rangle=6 D \mathbb{I} \delta(t),
\end{aligned}
$$

II denotes the $N \times N$ unit matrix. Finally, $w$ denotes the elementary Rouse rate and $D=\frac{1}{3} b^{2} w$ with $b$ being the Kuhn length.

As an example we mention that equations of motion for the smallest possible three arm star $\mathrm{BB}_{3}$ read as

$$
\begin{aligned}
\frac{d \mathbf{R}_{0}}{d t} & =-w\left(3 \mathbf{R}_{0}-\mathbf{R}_{1}-\mathbf{R}_{2}-\mathbf{R}_{3}\right)+\mathbf{f}_{0}, \\
\frac{d \mathbf{R}_{1}}{d t} & =-w\left(\mathbf{R}_{1}-\mathbf{R}_{0}\right)+\mathbf{f}_{1}, \\
\frac{d \mathbf{R}_{2}}{d t} & =-w\left(\mathbf{R}_{2}-\mathbf{R}_{\mathbf{0}}\right)+\mathbf{f}_{2}, \\
\frac{d \mathbf{R}_{3}}{d t} & =-w\left(\mathbf{R}_{3}-\mathbf{R}_{0}\right)+\mathbf{f}_{3},
\end{aligned}
$$

from which we see that the matrix $T$ reads

$$
T=\left[\begin{array}{cccc}
3 & -1 & -1 & -1 \\
-1 & 1 & 0 & 0 \\
-1 & 0 & 1 & 0 \\
-1 & 0 & 0 & 1
\end{array}\right] .
$$

Likewise for $\mathrm{B}\left(\mathrm{B}_{3}\right)_{3}$, we find

$$
T=\left[\begin{array}{cccccccccc}
3 & -1 & 0 & 0 & -1 & 0 & 0 & -1 & 0 & 0 \\
-1 & 2 & -1 & 0 & 0 & 0 & 0 & 0 & 0 & 0 \\
0 & -1 & 2 & -1 & 0 & 0 & 0 & 0 & 0 & 0 \\
0 & 0 & -1 & 1 & 0 & 0 & 0 & 0 & 0 & 0 \\
-1 & 0 & 0 & 0 & 2 & -1 & 0 & 0 & 0 & 0 \\
0 & 0 & 0 & 0 & -1 & 2 & -1 & 0 & 0 & 0 \\
0 & 0 & 0 & 0 & 0 & -1 & 1 & 0 & 0 & 0 \\
-1 & 0 & 0 & 0 & 0 & 0 & 0 & 2 & -1 & 0 \\
0 & 0 & 0 & 0 & 0 & 0 & 0 & -1 & 2 & -1 \\
0 & 0 & 0 & 0 & 0 & 0 & 0 & 0 & -1 & 1
\end{array}\right] .
$$


We solve Eq. (A.1) by diagonalizing matrix $T$ :

$$
\begin{aligned}
R & =S X, \\
\frac{d X}{d t} & =-w \Lambda X+G, \\
\Lambda & =S^{-1} T S, \\
G & =S^{-1} F,
\end{aligned}
$$

here $S$ is chosen such that $\Lambda$ is diagonal, i.e. such that the columns of $S$ are mutually orthogonal eigenvectors of $T$. The entries of $X$ are called Rouse vectors. It is convenient to normalize these eigenvectors to unity, in which case $S^{-1}=S^{T}$. In that case,

$$
\begin{array}{cl}
\langle G\rangle & =0, \\
\left\langle G(t) \cdot G(0)^{T}\right\rangle & =6 D \mathbb{\amalg} \delta(t) .
\end{array}
$$

Always one of the eigenvalues of $T$ is equal to zero, with corresponding normalized eigenvector having all entries equal to $1 / N$. The corresponding Rouse-vector $\mathbf{X}_{0}$ and random displacement vector $\mathbf{G}_{0}$ are given by

$$
\begin{aligned}
\mathbf{X}_{0} & =\frac{1}{\sqrt{N}} \sum_{k=1}^{N} \mathbf{R}_{k}, \\
\mathbf{G}_{0} & =\frac{1}{\sqrt{N}} \sum_{k=1}^{N} \mathbf{F}_{k} .
\end{aligned}
$$

Solving the equation of motion in this case, we find

$$
\begin{aligned}
& \mathbf{X}_{0}(t) \quad=\mathbf{X}_{0}(0)+\int_{0}^{t} d t^{\prime} \mathbf{G}_{0}\left(t^{\prime}\right), \\
& \left\langle\left[\mathbf{X}_{0}(t) \quad-\mathbf{X}_{0}(0)\right]^{2}\right\rangle=6 D t
\end{aligned}
$$

Notice that $\mathbf{X}_{0}$ is $\sqrt{N} \mathbf{R}_{c m}$, so the diffusion coefficient is $D_{R}=D / N$.

For the Rouse-vectors with non-zero eigenvalues, we obtain

$$
\mathbf{X}_{k}(t)=\mathbf{X}_{0}(0) e^{-w \lambda_{k} t}+\int_{0}^{t} d t^{\prime} e^{-w \lambda_{k}\left(t-t^{\prime}\right)} \mathbf{G}_{k}\left(t^{\prime}\right) .
$$

Noticing that $\left\langle\mathbf{X}_{k}(t) \cdot \mathbf{X}_{k}(t)\right\rangle=\left\langle\mathbf{X}_{k}(0) \cdot \mathbf{X}_{k}(0)\right\rangle$,we find

$$
\begin{aligned}
\left\langle\mathbf{X}_{k} \cdot \mathbf{X}_{k}\right\rangle & =6 D /\left(2 w \lambda_{k}\right)=b^{2} / \lambda_{k}, \\
\left\langle\left[\mathbf{X}_{k}(t)-\mathbf{X}_{k}(0)\right]^{2}\right\rangle & =\frac{2 b^{2}}{\lambda_{k}}\left(1-e^{-w \lambda_{k} t}\right),
\end{aligned}
$$


with these results we are now in the position to calculate blob mean square displacements.

For this purpose, notice that

$$
\mathbf{R}_{n}=\frac{1}{\sqrt{N}} \mathbf{X}_{0}+\sum_{k=1}^{N-1} S_{n k} \mathbf{X}_{k} .
$$

We then easily obtain

$$
\begin{aligned}
& \left\langle\left[\mathbf{R}_{n}(t)-\mathbf{R}_{n}(0)\right]^{2}\right\rangle \\
= & 6 D_{R} t+2 \sum_{k=1}^{N-1} S_{n k}^{2} \quad\left\langle\mathbf{X}_{k} \cdot \mathbf{X}_{k}\right\rangle\left(1-e^{\left.-\omega \lambda_{k} t\right)} .\right.
\end{aligned}
$$

The example of $\mathrm{BB}_{3}$ given above has eigenvalues $\lambda_{k}=0,1,1,4$. The corresponding $S$ matrix reads

$$
S=\left[\begin{array}{cccc}
\frac{1}{2} & 0 & 0 & \frac{1}{2} \sqrt{3} \\
\frac{1}{2} & \frac{1}{2} \sqrt{2} & 0 & 0 \\
\frac{1}{2} & -\frac{1}{2} \sqrt{2} & 1 & 0 \\
\frac{1}{2} & 0 & 0 & 1 .
\end{array}\right]
$$

Similarly, the $S$ for $\mathrm{B}\left(\mathrm{B}_{3}\right)_{3}$ can be calculated. 



\title{
Simulations of Symmetric Star Polymers with Different Arm Lengths
}

\begin{abstract}
Melts of three-armed polymers have been simulated using a coarse grained model parameterized by atomistic simulations of polyethylene. The bonds between the highly coarse grained, and hence soft, polymer beads are explicitly prevented from crossing by the TWENTANGLEMENT algorithm. Three melts of symmetric stars, differing in the lengths of the arms, are compared against five melts of linear polymers with comparable dimensions, to assess the impact of the branched architecture on the self-diffusion and the bulk rheological properties. A satisfactory quantitative agreement with experiments is observed for the diffusion coefficients and zero-shear viscosities of the linear polymers. The power-law relation between viscosity and molecular mass is indicative of the transition regime between Rouse and reptation dynamics. The star polymers, in stead, follow an exponential mass-viscosity relation and show qualitative agreement with the storage and loss moduli for stars with far longer arms. The stress relaxation $d y-$ namics of the stars is also compared with a theoretical analysis in terms of Rouse modes.
\end{abstract}

\subsection{Introduction}

Melts of star polymers behave differently from melts of linear polymers made of identical monomers. They even obey distinctly different scaling laws. A well-known example is the relation between the zero-shear viscosity and the molecular mass: for linear polymers the 
viscosity scales as a power of the total mass [27,28], while for symmetric star polymers the viscosity grows exponentially with the mass of an arm $[78,79]$. This marked difference is a consequence of the polymer dynamics at the microscopic level. Long linear polymers perform reptation dynamics, i.e. they move predominantly along their own contour path, confined to the tube formed by their neighbours [36,63]. Star polymers with $f$ long arms, where $f$ is also known as the functionality, will have their arms confined to $f$ distinct tubes for most of the time, thereby ruling out reptation dynamics. In stead, stars and other branched polymers are believed to move by retracting an arm from its tube through thermal motion, followed by extension of this arm into a new tube [80-84], which strongly reduces the dynamics of stars $[85,86]$. Insights into the dynamical properties of polymer melts, and the microscopic processes giving rise to the macroscopic properties, are gained in mutually supplementary studies by experiment, theory and computer simulation $[62,87]$. The dynamics of branched polymers is of particular interest due to their wide usage in industry [88-91].

The long polymer or arm lengths required to see reptation or retraction dynamics, respectively, pose a challenge to computer simulations. Ideally, one would like to simulate polymers at the all-atom level by Molecular Dynamics (MD) to obtain a nearly exact agreement between simulations and experiments, but the long length and time scales involved make this computationally very demanding simulations [92]. The group of Tanner reported united-atom simulations of $\mathrm{C}_{100} \mathrm{H}_{202}$ polyethylene (PE) isomers, using non-equilibrium techniques to overcome the slow dynamics, in their study on the influence of star architecture on the shear thinning characteristics [7]. To reduce the computational burden, one often lumps together several backbone atoms and their adjacent hydrogen atoms or small side groups into 'beads'. These beads are typically modelled as Lennard-Jones (LJ) spheres, connected by finitely extensible nonlinear elastic (FENE) bonds [93] In this bead-spring polymer model, the LJ and FENE potentials are tuned to guarantee that the chains can not cross each other, thus retaining the key feature of polymer dynamics. The well-known Kremer-Grest model for linear polymers was also applied, by its developers, to study the structural features of star polymers, like the mass distribution, and their internal relaxation dynamics [94,95]. Zhou and Larson used a slightly modified model, including a bending potential to create a less flexible chain, to study the predicted hopping dynamics of the branching point when an arm has spontaneously retracted [96]. Bačová et al. used the same model to compare branch 
point dynamics in stars with free arm ends and stars with fixed arm ends [97]. Continuing along the coarse-graining path of sacrificing chemical details to gain access to longer time and length scales, one may group ever more backbone atoms (and their side groups) together into mesoscopic 'blobs'. The effective repulsive interaction between these blobs, as deduced from atomistic MD simulations [44,47], becomes softer with increasing blob mass, while the bonds between the blobs become longer. Both trends are detrimental to the automatic prevention of bond crossings in the simulations, hence alternative schemes have to be introduced to re-instate entanglement effects. In slip-link models, the chains of particles are threaded through a sequence of rings which are connected to fixed points in space by rigid constrains or harmonic potentials $[73,98]$. The rings restrict the sideways motions of the chain, but not the reptational sliding of the chain through the rings; a series of rules govern the creation of new rings when loops or dangling ends grow too large, while vacated rings are removed. Masubuchi et al. used a slip-link model to study the viscoelastic properties of a melt of star polymers, and the impact of branch point hopping on stress relaxation [99]. Padding and Briels developed the TwENTANGLEMENT algorithm $[44,45]$ that explicitly detects imminent bond crossings and inserts entanglement points to prevent the bonds from actually crossing. The equations of motion of the blobs and entanglement points are coupled by mutual interactions; the chains are allowed to slip past an entanglement point, and several mechanisms allow for disentanglement. We have recently extended this algorithm to handle branched molecules. The first simulations of a melt of symmetric three-armed stars already clearly highlighted the impact of the polymer architecture on the melt properties: the short-armed stars diffuse like linear polymers of equal mass, but their rheological properties resemble those of a linear polymer of equal end-to-end span [46]. In highly idealized lattice models, such as the bond fluctuation model (BFM), the polymer beads are confined to lattice points; the beads are displaced by Monte Carlo moves that explicitly forbid bond crossing and double occupancies of lattice sites [100,101]. Pakula used lattice models to study the structure and relaxation dynamics of stars in melts, observing a hopping motion of star centers of mass $[102,103]$. The lattice model simulations of three-armed stars by Brown and Szamel are inconclusive on the power-law versus exponential scaling of the diffusion coefficient with the mass of the arm [104]. Several of the aforementioned studies also present results on asymmetric stars, and/or on more complex branched architectures $[7,96,97,99]$. 
In this work, we will use the TWENTANGLEMENT algorithm. Application of this method to melts of linear PE chains of various lengths yielded good agreement with experimental studies [45]. We have recently extended the technique to branched molecules, and successfully applied the algorithm to study a melt of PE stars with short arms [46]. Here, we extend our previous study to symmetric three-armed stars with longer arms, thereby allowing the arms to get more thoroughly entangled. The dynamical and rheological properties of three melts are studied and compared against five melts of matching linear polymers. A global overview of the TWENTANGLEMENT algorithm, the simulation set-up and the applied analysis methods is provided in Section 3.2. The simulation results are presented and discussed in Section 3.3, where the linear polymers precede the star polymers to facilitate the comparison. Our main conclusions are summarized in Section 3.4.

\subsection{Model and methods}

\subsubsection{Coarse grained polymers}

In this study, we used the coarse grained TWENTANGLEMENT model. Originally developed by Padding and Briels $[44,45]$ for the simulation of linear polymers, the algorithm was recently extended by us [46] to study polymers with more complex architectures. The polymers are composed of coarse grained particles (or blobs), each representing 20 consecutive backbone carbons of a polyethylene (PE) chain.

Assuming complete separation of time scales between the eliminated and retained degrees of freedom, i.e. the positions $\mathbf{R}^{n}=\left\{\mathbf{R}_{1}, \ldots, \mathbf{R}_{n}\right\}$ of the $n$ blobs, and neglecting effects of inertia, the time evolution of the position of the $i^{\text {th }}$ blob is described by the first order Langevin equation

$$
d \mathbf{R}_{i}=-\frac{1}{\xi} \nabla_{i} \Phi d t+\sqrt{\frac{2 k_{B} T d t}{\xi}} \Theta_{i} .
$$

The friction coefficient $\xi$ in the first term and the random displacements in the second term represent the influence of the eliminated fast degrees of freedom on the retained slow degrees of freedom, with $k_{B}$ the Boltzmann constant, $T$ the absolute temperature, and where $\Theta_{i}$ denotes a Markovian stochastic vector of zero mean and unit variance, devoid of correlations 
between its components and between particles, and without memory. The potential of mean force (PMF) is defined as $\Phi\left(\mathbf{R}^{n}\right)=-k_{B} T \ln P\left(\mathbf{R}^{n}\right)$, where the distribution function $P$ of blob center of mass positions has previously been determined by grouping together 20 consecutive united atom $\mathrm{CH}_{2}$ particles in molecular dynamics simulations of a $\mathrm{C}_{120} \mathrm{H}_{242}$ melt $[44,105]$. For practical reasons, the PMF has been approximated as a sum of contributions,

$$
\Phi\left(\mathbf{R}^{n}\right)=\sum_{i} \phi^{\text {bond }}\left(l_{i, i+1}\right)+\sum_{i} \phi^{\text {ang }}\left(\theta_{i, i+1, i+2}\right)+\sum_{i j} \phi^{\mathrm{nb}}\left(R_{i j}\right)
$$

with $\phi^{\text {bond }}$ the attractive bond potential between two consecutive blobs at a bond length $l_{i, i+1}=\left|\mathbf{R}_{i+1}-\mathbf{R}_{i}\right|, \phi^{\text {ang }}$ the bending potential between three consecutive blobs at an angle $\theta_{i, i+1, i+2}$, and $\phi^{\text {nb }}$ the repulsive non-bonded potential between any pair of blobs. We refer the reader to our previous paper [46] for the functional forms and the parametrizations of these interactions.

\subsubsection{Uncrossability constraints}

As mentioned in the Introduction, when the number of backbone carbons lumped into a blob increases, the interactions between the blobs become softer and softer. The soft interactions between $\mathrm{C}_{20}$ blobs do not prevent the $\mathrm{CG}$ polymers from crossing each other; this fundamental feature of polymers, with far reaching implications for the properties of a melt, is lost in the coarse-graining procedure. In order to restore proper polymer dynamics, these unrealistic bond crossing events are detected and prevented in the TWENTANGLEMENT algorithm. The crossing of the $i$ to $i+1$ bond with the $j$ to $j+1$ bond is detected by a change in sign, during the time step $d t$, of the triple product

$$
V_{i j}=\left[\left(\mathbf{R}_{i+1}-\mathbf{R}_{i}\right) \times\left(\mathbf{R}_{j+1}-\mathbf{R}_{j}\right)\right] \cdot\left(\mathbf{R}_{i}-\mathbf{R}_{j}\right)
$$

formed by the two bond vectors and their connecting vector. If the two bonds cross, an 'entanglement point' $\mathbf{X}$ is introduced at the (approximate) position of the crossing event. The position of this entanglement point is updated every time step, using the procedure outlined below, until the two bonds disentangle; this annihilation event is detected by a procedure similar to that used to detect their creation.

The collection of $n$ blob positions and a variable number of entanglement points is advanced in time by the following sequence of steps. For a given configuration, the potential 
energy and the forces are calculated by means of Eq. (3.2), with the one exception that the bond length is not calculated as the direct distance between two consecutive blobs but as the contour length of the (meandering) bond connecting these blobs:

$$
\sum_{i} \phi^{\text {bond }}\left(l_{i, i+1}\right) \rightarrow \sum_{i} \phi^{\text {bond }}\left(L_{i, i+1}\right)
$$

where

$$
L_{i, i+1}=\left|\mathbf{R}_{i}-\mathbf{X}_{1}^{i, i+1}\right|+\left|\mathbf{X}_{1}^{i, i+1}-\mathbf{X}_{2}^{i, i+1}\right|+\ldots+\left|\mathbf{X}_{p}^{i, i+1}-\mathbf{R}_{i+1}\right|
$$

with $\mathbf{X}_{1}^{i, i+1}, \ldots, \mathbf{X}_{p}^{i, i+1}$ denoting the $p$ entanglements along the bond connecting $\mathbf{R}_{i}$ to $\mathbf{R}_{i+1}$. The positions of the entanglement points are then determined by numerically minimizing the total binding potential energy with respect to the entanglement points, subject to the given blob positions. The resulting bond forces on the blobs, in combination with the angular and non-bonded forces, are used to update the blob positions by means of Eq. (3.1). As the last step in the cycle, the system is scanned to detect creations of new entanglements and annihilations of old entanglements. Whereas the detection of bond crossing has been explained in Eq. (3.3) using straight blob-to-blob paths, the employed algorithm detects crossings of the meandering paths between the blobs by applying Eq. (3.3) to all straight segments connecting successive points (by using entanglement positions as well as blob positions) along a polymer chain. Since real polymers can slide past an entanglement point, the detection step also allows entanglement points to slip over a blob, e.g. $\mathbf{X}_{p}^{i, i+1}$ becoming $\mathbf{X}_{1}^{i+1, i+2}$. Entanglements slipping over the last bead of a chain are annihilated. For details on the implementation of the Twentanglement algorithm, we refer the reader to Padding and Briels $[44,45]$.

When applied to three-armed star polymers, TWENTANGLEMENT must be extended in two ways. A minor change is that the angular potential centered on the branching point reaches its minima at $\theta= \pm 120^{\circ}$, rather than at $\theta=180^{\circ}$, as well as being stiffer than the bending potential centered on a blob in a linear chain. A major change is required to enable the slip of an entanglement point over a branching point, as this move now allows for more complex final states than the slip of an entanglement point over a blob in a linear chain. In our previous paper [46] we presented and tested a pragmatic solution to this complication.

The simulations were performed in cubic boxes with periodic boundary conditions, with the densities of all melts fixed at $\rho=0.761 \mathrm{~g} / \mathrm{cm}^{3}$. The number of polymers in the box varied 
from 100 for the shortest polymer, $\mathrm{B}_{9}$, to 80 for the longest chains, $\mathrm{B}_{19}$ and $\mathrm{B}\left(\mathrm{B}_{6}\right)_{3}$. Previous studies on linear polymers established an entanglement length of about 6 beads [45]. A friction coefficient $\xi=32 \mathrm{ps}^{-1}$ was used for all polymers. The thermostat implicit in Eq. (3.1) maintained a temperature of $450 \mathrm{~K}$. The timestep was $0.4 \mathrm{ps}$. Each melt was equilibrated as thoroughly as possible (see Section 3.3), followed by a production run covering about $12 \mu$ s.

\subsubsection{Analysis methods}

The dynamics of polymers in the melt will be quantified by the diffusion coefficient, as extracted from the mean square displacement

$$
D(t)=\frac{1}{6 t}\left\langle\left|\mathbf{R}_{i}^{c m}(t)-\mathbf{R}_{i}^{c m}(0)\right|^{2}\right\rangle,
$$

with $\mathbf{R}_{i}^{c m}(t)$ the center of mass position of the $i^{\text {th }}$ chain at time $t$, and the pointy brackets denoting an average over all reference times and molecules.

Stress relaxation moduli are calculated from equilibrium simulations by means of the auto correlation

$$
G(t)=\frac{V}{6 k_{B} T} \sum_{\alpha \beta}\left\langle\sigma_{\alpha \beta}(t) \sigma_{\alpha \beta}(0)\right\rangle,
$$

where the sum runs over the six off-diagonal elements and the stress tensor is given by

$$
\sigma_{\alpha \beta}=-\frac{1}{V} \sum_{\langle i, j\rangle}\left(Y_{i, \alpha}-Y_{j, \alpha}\right) f_{i j, \beta} .
$$

Here $Y_{i, \alpha}$ denotes the $\alpha$-component of the position $\mathbf{Y}_{i}$ of the $i^{\text {th }}$ entity - either a blob or an entanglement point - in the box of volume $V, f_{i j, \beta}$ is the $\beta$-component of the force exerted on this entity by entity $j$, and the sum runs over all entity pairs. A typical example of a calculated stress auto correlation is shown in Fig. 3.1. The plotted markers are randomly selected from the calculated $G(t)$, showing all data points at low times but reaching a constant density for large times, with red points representing positive values and blue points for negative values. The distribution of these points gives a clear impression of the relatively low noise level obtainable by the TWENTANGLEMENT method and of $G(t)$ essentially having decayed to zero for times exceeding a microsecond. The drawn line was obtained by block-averaging the raw data:

$$
\bar{G}(t)=\int_{-\Delta}^{\Delta} G(t+\tau) w(\tau) d \tau,
$$




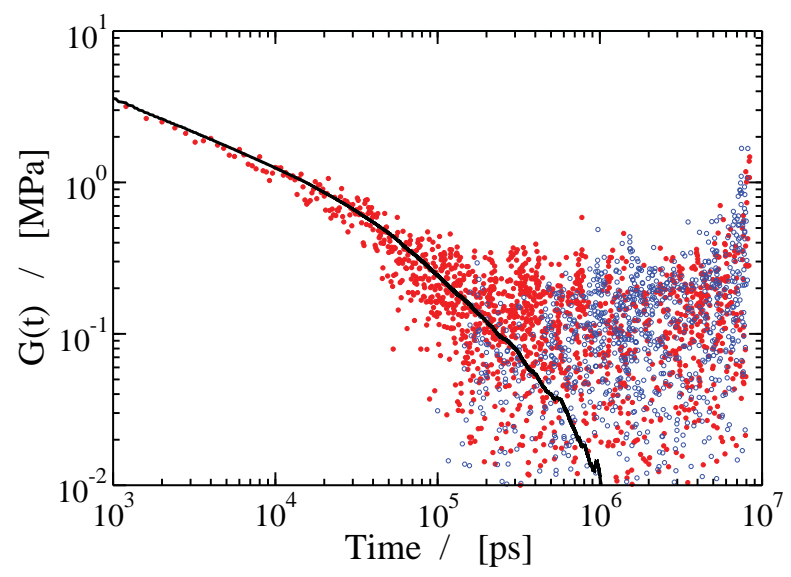

Figure 3.1: Stress relaxation modulus for $\mathrm{B}\left(\mathrm{B}_{6}\right)_{3}$ on a double logarithmic scale. The markers present the raw data, red dots for positive values of $G(t)$ and blue circles for negative values. The solid line is the block average $\bar{G}(t)$ calculated using Eq. (3.9).

where $w$ is a normalized weight function on the interval $[-\Delta, \Delta]$. We found that $w(\tau) \sim 1 / \tau$, i.e. $w(\tau) d \tau \sim d \ln \tau$, gives good results.

Having established the stress relaxation modulus, the zero-shear viscosity is calculated by the Green-Kubo integral

$$
\eta_{0}=\int_{0}^{\infty} G(t) d t
$$

while the Fourier transforms

$$
\begin{aligned}
G^{\prime}(\omega) & =\omega \int_{0}^{\infty} \sin (\omega t) G(t) d t \\
G^{\prime \prime}(\omega) & =\omega \int_{0}^{\infty} \cos (\omega t) G(t) d t
\end{aligned}
$$

yield the storage and loss modulus, respectively. In the calculation of these integrals, the raw data were used up to the first appearances of negative values, while for larger times $G(t)$ was approximated by a single decaying exponential fitted to the block averaged curve $\bar{G}(t)$. We note that this smoothed tail contributes only $\sim 5 \%$ to the zero-shear viscosity, rendering the final results insensitive to the details of the fit.

44 


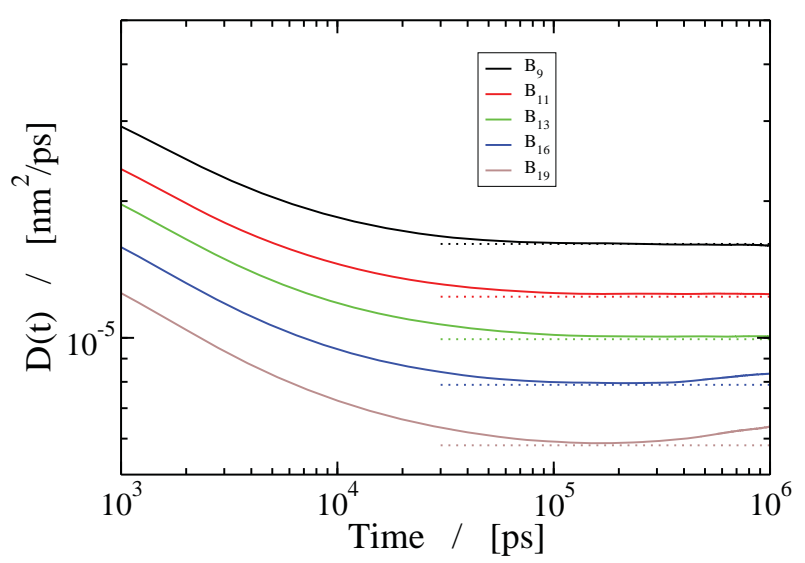

Figure 3.2: Time-dependent diffusion coefficients for linear polymers of various lengths, as extracted from the mean squared displacement of the polymer center of mass.

\subsection{Results and Discussion}

In this section the dynamical properties of melts of star polymers will be compared against the dynamical properties of melts of linear polymers. The latter will be discussed first, to set the background. We note that advances in hardware and software have made it possible to simulate the linear polymers over considerably longer time scales than in previous TWENTANGLEMENT studies [45]. This has now made possible a direct evaluation of the dynamical properties of the longer chains, without resorting to extrapolation techniques.

\subsubsection{Linear polymers}

\subsubsection{Diffusion}

From theory and experimental observations it is known that the diffusion coefficient in a melt of linear polymers decays with increasing molecular mass $M$, with $D \propto M^{-1}$ for short polymers in the Rouse regime $\mathrm{i}$ and $D \propto M^{-2}$ for long polymers in the reptation regime $[25,28,34,63]$. Figure 3.2 shows the time-dependent center-of-mass diffusion coefficients as extracted from the simulations by Eq. (4.5). After a transient decay on the nanosecond time scale, the functions are seen to reach a plateau value that defines the long time diffusion 


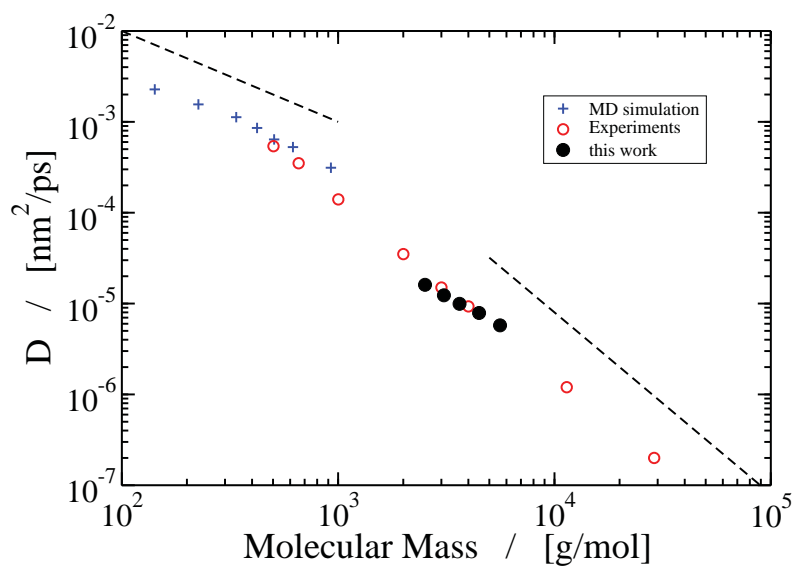

Figure 3.3: Long time diffusion coefficients of linear polymers as a function of molecular mass. The black dots are the results of this study, the red circles represent experimental data from Pearson et al. [27], and the red pluses show molecular dynamics simulation results obtained by Mondello et al. [106, 107]. The dashed lines illustrate the slopes expected for the Rouse regime (left, $D \propto M^{-1}$ ) and the reptation regime (right, $D \propto M^{-2}$ ).

coefficient $D$. For the longest chain, which does not reach a clear plateau, $D$ is approximated by the minimum value of the $D(t)$ curve. The terminal diffusion coefficients are plotted in Fig. 3.3 against the molecular weight, showing a satisfactory quantitative agreement with the experimental data by Pearson et al. [27]. Fitting the data with a power law yields an exponent of -1.27 , indicating that the chains under investigation are in the transition region between the Rouse and reptation regimes.

\subsubsection{Stress relaxation moduli}

Stress relaxation moduli for five linear polymers are collected in Fig. 3.4. The curves almost coalesce for short times and fan out for large times, with the longer polymers relaxing more slowly than the shorter ones. The resulting viscosities at vanishing shear rate are plotted in Fig. 3.5, supplemented with the zero-shear viscosities of three shorter chains, downto $\mathrm{B}_{2}$. The results indicate that the simulations are in the transition regime between Rouse and reptation dynamics. The power of $1.71 \pm 0.05$ extracted from the first four points is appreciably lower 


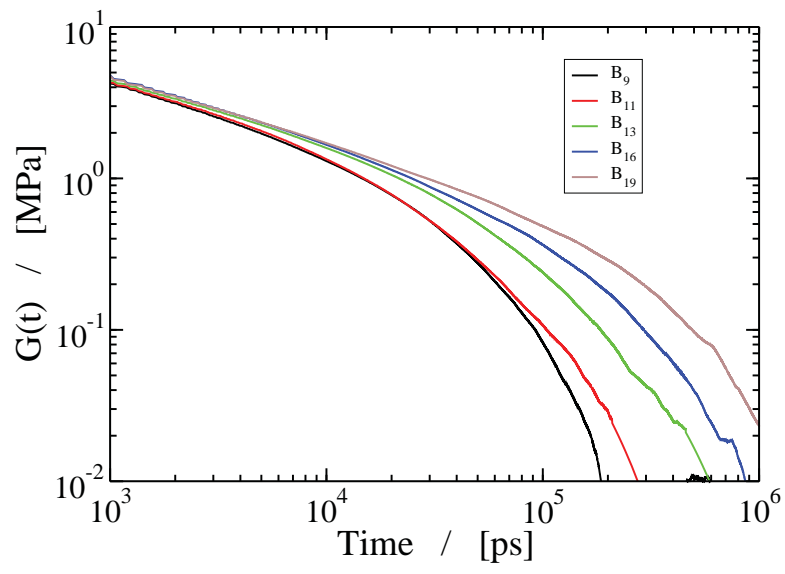

Figure 3.4: Block-averaged stress relaxation moduli for five melts of linear polymers of various chain lengths.

than the power of $2.4 \pm 0.2$ extracted from the next four points, confirming that the simulated melts are in the transition regime. Comparison with the experimental viscosities of Pearson et al. [27] reveals a good quantitative agreement. Unlike for the diffusion coefficient, the location and width of the transition regime of the simulated melts appear to agree with those of the experimental melts.

\subsubsection{Three-armed stars}

It is well known that the properties of a melt are strongly affected by the architecture of the constituent polymers. $[6,7,12-14]$ In our previous work, on the development and validation of TWENTANGLEMENT for non-linear chains, we simulated a melt of three-armed stars with short arms, $\mathrm{B}\left(\mathrm{B}_{3}\right)_{3}$. Here, we study symmetric three-armed stars with longer arms approaching the entanglement length, up to $\mathrm{B}\left(\mathrm{B}_{6}\right)_{3}$. The dynamical properties will be compared to those of matching linear polymers; for a $\mathrm{B}\left(\mathrm{B}_{n}\right)_{3}$ star, these are the linear polymer of the same mass $\mathrm{B}_{3 n+1}$ and the linear polymer with the same maximum end-to-end span, $\mathrm{B}_{2 n+1}$. 


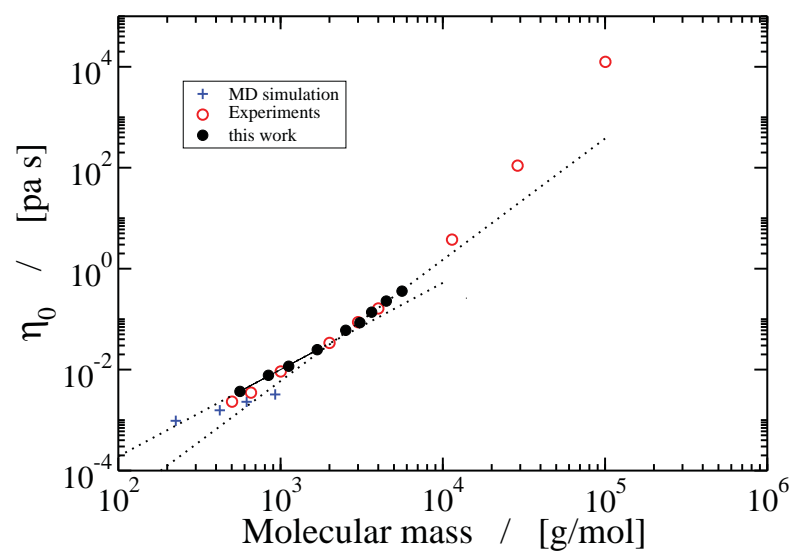

Figure 3.5: Zero shear viscosities of melts of linear polymers as a function of the molecular mass. The black dots are the results of this study, the red circles represent experimental data from Pearson et al. [27], and the red pluses show simulation results obtained by Mondello et al. [106] using molecular dynamics. The dashed lines highlight the experimental scaling laws at low mass $\left(\eta \propto M^{1.8}\right)$ and high mass $\left(\eta \propto M^{3.5}\right)$.

\subsubsection{Diffusion}

The time-dependent diffusion coefficients for three melts of stars polymers are depicted in Fig. 3.6. Like for the linear polymers, $D(t)$ initially decays before leveling off, where the height of the plateau again decreases with increasing mass. To make the comparison with the linear polymers more explicit, the three curves are redrawn in separate subplots of Fig. 3.7 along with the $D(t)$ of the matching linear polymers. It is readily observed that, of the three polymers per subfigure, the linear polymer of matching end-to-end span has the largest diffusion coefficient. For small times, the diffusion coefficients of the stars closely resemble those of the linear polymers of equal mass. The smallest star, like the smaller $\mathrm{B}\left(\mathrm{B}_{3}\right)_{3}$ simulated in our previous study, even matches the $D(t)$ of the equal mass linear polymer over the entire time range. With increasing arm length, however, the effect of the topological difference becomes apparent as the stars converge to smaller terminal diffusion coefficients than the matching equal mass linear polymers. 


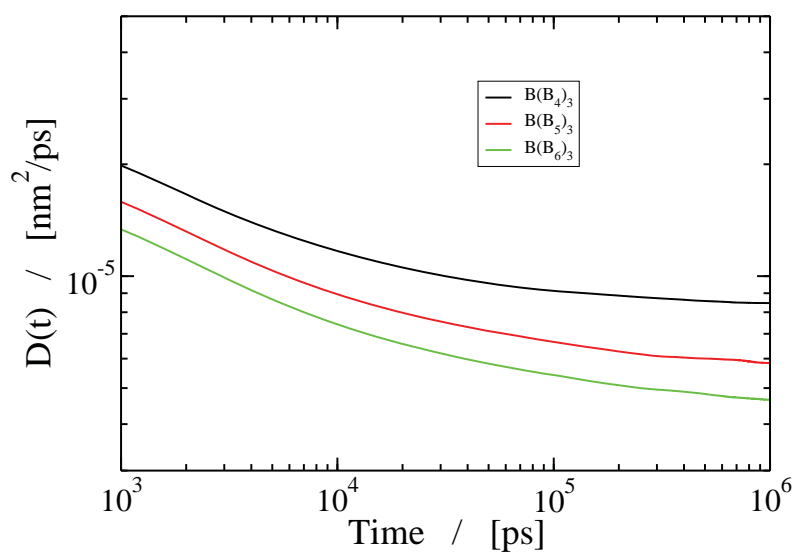

Figure 3.6: Time-dependent diffusion coefficients for three melts of three-armed star polymers.

\subsubsection{Stress relaxation moduli}

The stress relaxation moduli of four star polymer melts are shown in Fig. 3.8. At first glance these curves appear fairly similar to those shown in Fig. 3.4 for the linear polymers: for small times the curves nearly overlap, while for large times the curves spread out with the largest star relaxing the slowest. To facilitate the comparison, the stress relaxation functions of the three stars are plotted in the three subfigures to Fig. 3.9 together with the curves of their matching linear polymers. For the smallest star, $\mathrm{B}\left(\mathrm{B}_{4}\right)_{3}$, the $G(t)$ closely follows that of the linear polymer of equal maximum span, $\mathrm{B}_{9}$. With increasing arm length, the tail of $G(t)$ decays slower for the star than for the linear polymer of equal span, and creeps toward the $G(t)$ of the polymer of equal mass. This slackening of the relaxation process at late times is attributed to the architecture of the star. The largest star polymer in Fig. 3.8, with 7 beads per arm, has been excluded from this and following comparisons against linear polymers, because its very slow dynamics makes it difficult to asses whether the small plateau appearing in the tail of its $G(t)$, beyond $\sim 5 \cdot 10^{5}$ ps, represents a true shoulder or merely emerges as an artefact of insufficient sampling.

In Fig. 3.9 shear relaxation moduli of stars are compared with those of linear chains. In general the star relaxes at time scales between those of the longest end-to-end and the total 


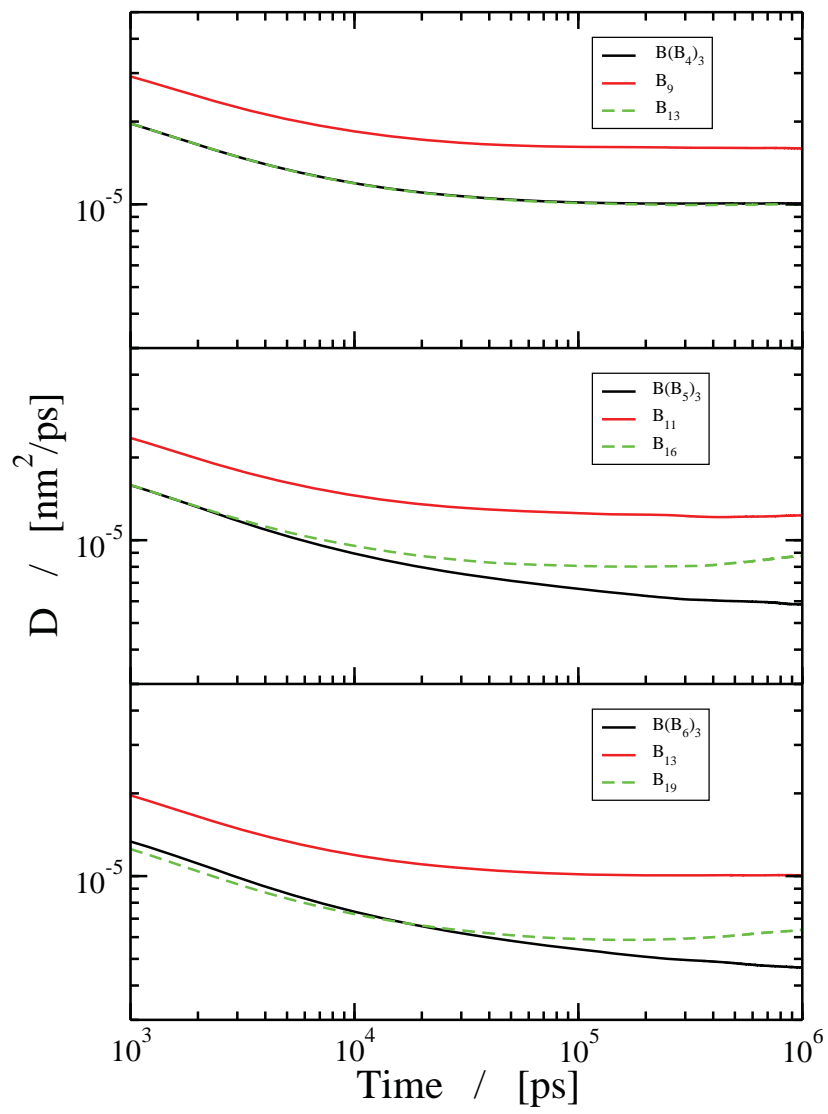

Figure 3.7: Time-dependent diffusion coefficients for three melts of three-armed star polymers (black solid lines), compared against the diffusion of linear polymers of equal mass (green dashed lines) and of equal end-to-end span (red solid lines).

mass linear polymer. With increasing mass, its longest time gradually approaches that of the total mass linear. This leads to qualitatively different behaviour of the relaxation moduli of the stars and linear chains. The storage and loss moduli of the star polymers are presented in Fig. 3.10. At the low frequency side, the $G^{\prime} \propto \omega^{2}$ and $G^{\prime \prime} \propto \omega$ tails are seen to shift to lower frequencies with increasing molecular mass, reflecting an increase of the zero-shear viscosity [29]. At the high frequencies, the storage and loss moduli of the three stars nearly coalesce 


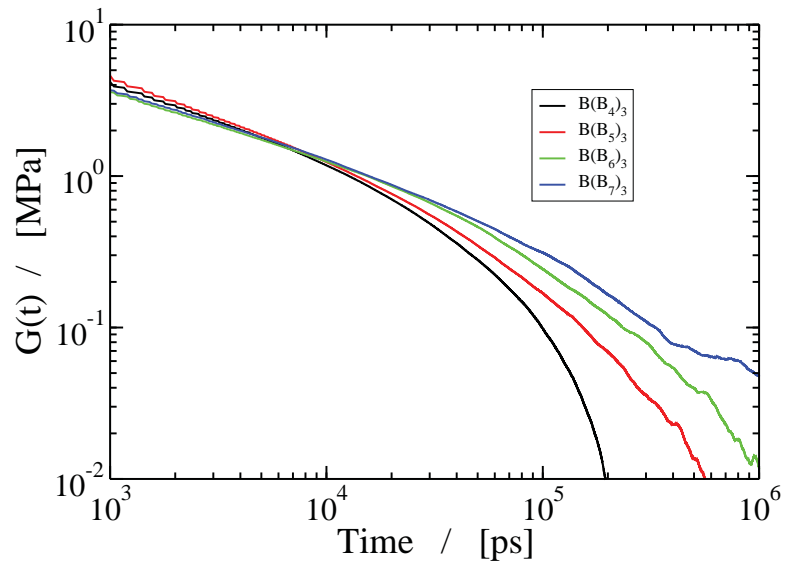

Figure 3.8: Stress relaxation moduli for four melts of three-armed star polymers.

to straight lines in the double logarithmic plot, with power laws fitted to the individual curves yielding exponents in the 0.35 to 0.55 range. The storage and loss moduli of the star polymers are being compared against the moduli of the linear polymer of equal mass in Fig. 3.11. For every melt, the frequencies have been scaled by the frequency at the cross-over point, $\omega_{c} \sim 10^{-4} \mathrm{ps}^{-1}$, and the moduli have been scaled by the modulus at the cross-over point, $G^{\prime}\left(\omega_{c}\right)=G^{\prime \prime}\left(\omega_{c}\right) \sim 1 \mathrm{MPa}$. The plot shows that the rescaled moduli of the smallest star closely adhere in the two decades following the cross-over point to those of the linear polymer of equal mass, suggesting that the polymer topology barely affects the dynamics of these short-armed stars in this frequency range. With increasing arm length, the moduli of the stars are seen to deviate from the moduli of the linear polymers in the two decades following the cross-over. The main feature to be noted is the 'wedge' between the storage and loss moduli, i.e. the gradual growth with increasing frequency of the difference between the solid and dashed curves. This wedge is observed for the melts of linear polymers, and opens up with approximately the same angle for all three melts in Fig. 3.11. An identical wedge is seen for the star with the shortest arms, $\mathrm{B}\left(\mathrm{B}_{4}\right)_{3}$. For the stars with one additional bead per arm, however, the angle has reduced considerably. The wedge has essentially closed for the largest star, $\mathrm{B}\left(\mathrm{B}_{6}\right)_{3}$, where the storage and loss moduli are seen to nearly coalesce for the first decade and a half following the cross-over. (Note that for this melt the curves were rescaled 


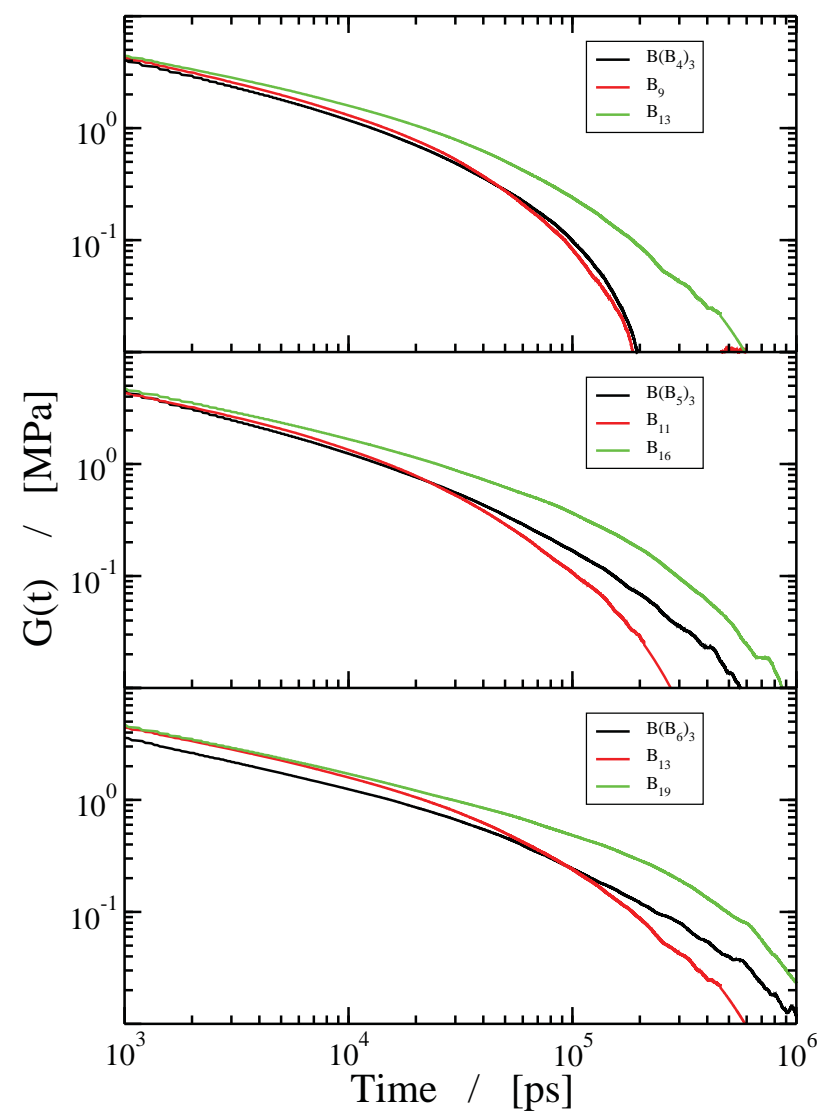

Figure 3.9: Stress relaxation moduli for three melts of three-armed star polymers (solid black lines), compared against the moduli of linear polymers of equal mass (red solid lines) and of equal end-to-end span (green dashed lines).

relative to the cross-over point at the low frequency end of the overlap region). Although the polymers are evidently too short to attain the large numbers of entanglements required for reptation dynamics, the simulations nevertheless agree qualitatively with experiments on long polymers in yielding a wider wedge for the linear polymers than for the star polymers $[12,14]$. In the experimental studies the storage and loss moduli of star polymers continue to rise after the cross-over point, while for linear polymers $G^{\prime}$ crosses the local maximum of $G^{\prime \prime}$. The 

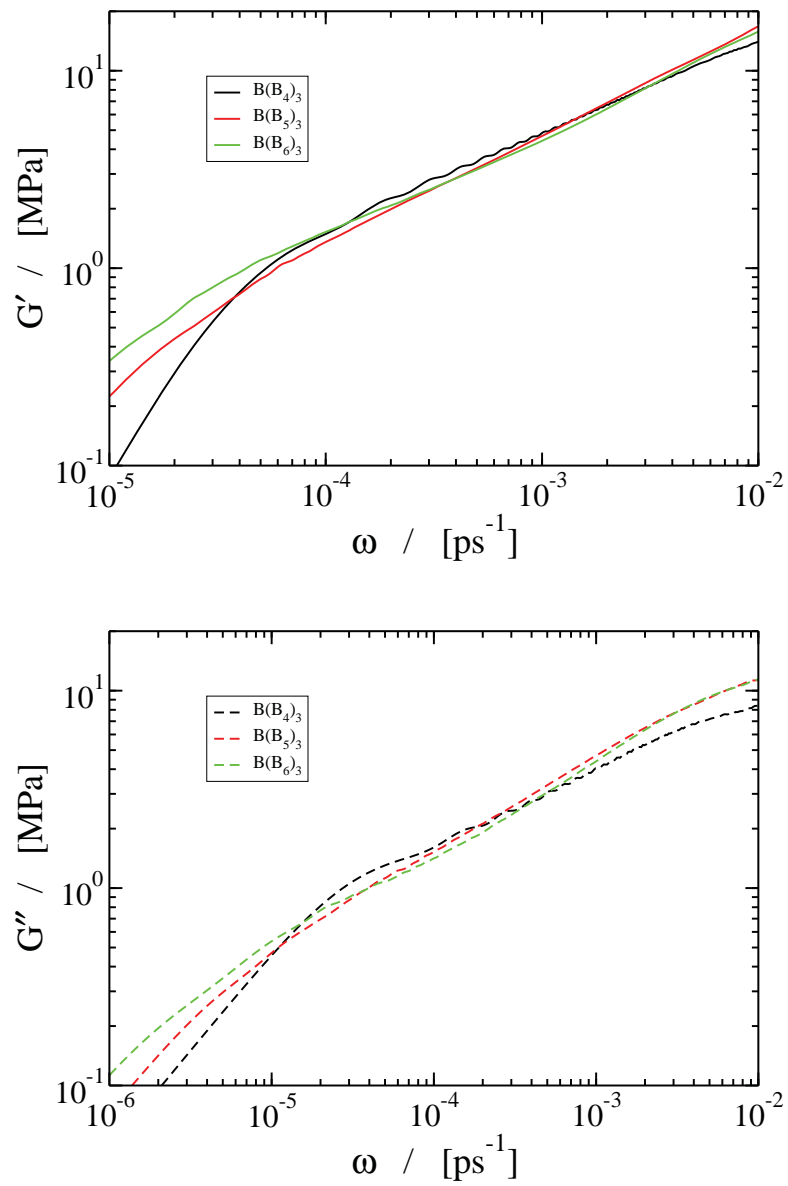

Figure 3.10: Storage (top) and loss (bottom) moduli for melts of three-armed star polymers.

steeper slopes in Fig. 3.11 - and the absence of a local maximum in $G^{\prime \prime}$ for the linear polymers - reflect the limited length of the simulated polymers.

The viscosities calculated from the stress relaxation curves in Fig. 3.8 are plotted in Fig. 3.12 against the molecular mass $M$. Also included is the small $\mathrm{B}\left(\mathrm{B}_{3}\right)_{3}$ star of our previous study [46]. Fitting the viscosities of these five melts with a power law yields the red line in Fig. 3.12, with a power of $1.6 \pm 0.2$. Whereas the nine melts of linear polymers are clearly seen in Fig. 3.5 to follow a power law with a markedly higher power, the star poly- 


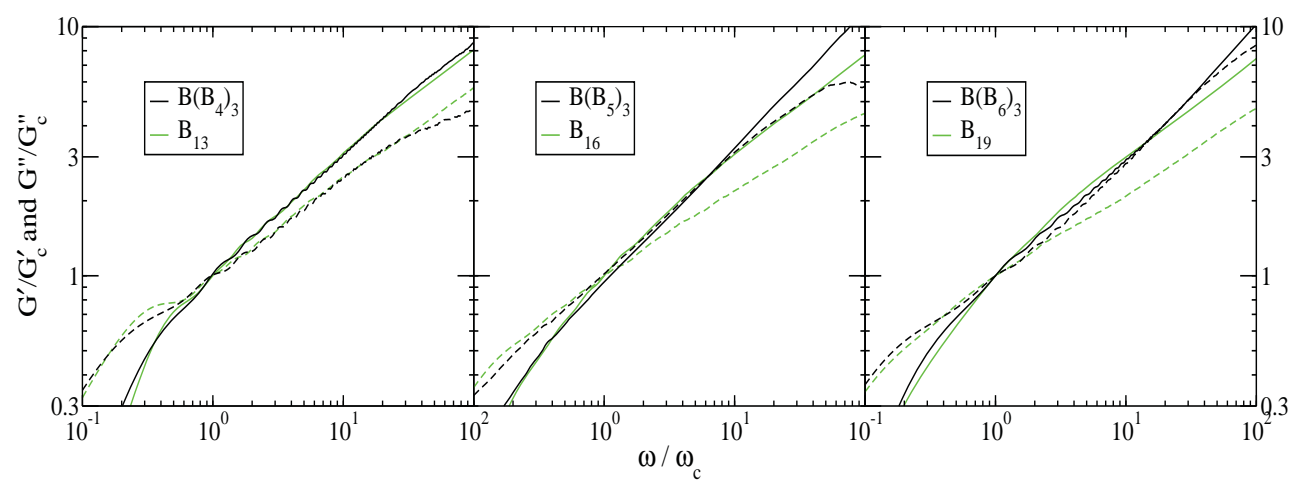

Figure 3.11: Storage (solid lines) and loss moduli (dashed lines) for melts of three-armed star polymers (black) and linear polymers of equal mass (green). For comparison purposes, the frequencies and moduli have been scaled by the frequency and moduli at their cross-over points, $\omega_{c}, G_{c}^{\prime}$ and $G_{c}^{\prime \prime}$ respectively, of the respective melts.

mers reveal a systematic deviation from a power law in Fig. 3.12. The viscosities are better fitted by an exponential, $\eta \propto e^{v M}$ with $v=(1.3 \pm 0.1) \cdot 10^{-4} \mathrm{~mol} / \mathrm{g}$, as shown by the green line in Fig. 3.12. Assuming an entanglement mass $M_{e}$ of six beads [45] yields $\eta \propto e^{v^{\prime}\left(M_{a} / M_{e}\right)}$ with $M_{a}$ the mass per arm; the coefficient $v^{\prime} \approx 0.07$ underestimates the experimental value of 0.47 [12]. Experiments on stars with many and/or long arms point at a power-exponential scaling law [12], but fits of the simulation viscosities with a function of this type converge to a negative power, in contradiction to the experimental and theoretical results. We note that in the calculation of the viscosity of the largest star, $\mathrm{B}\left(\mathrm{B}_{7}\right)_{3}$, the integrated exponential tail of its stress relaxation function was fitted to the exponentially decaying regime of the block-averaged $\bar{G}(t)$ preceding the suspect plateau region. Since the resulting viscosity matches well with an exponential fitted through the viscosities of the four shorter polymers, the plateau is more likely a consequence of insufficient sampling than a rheological feature of the system. 


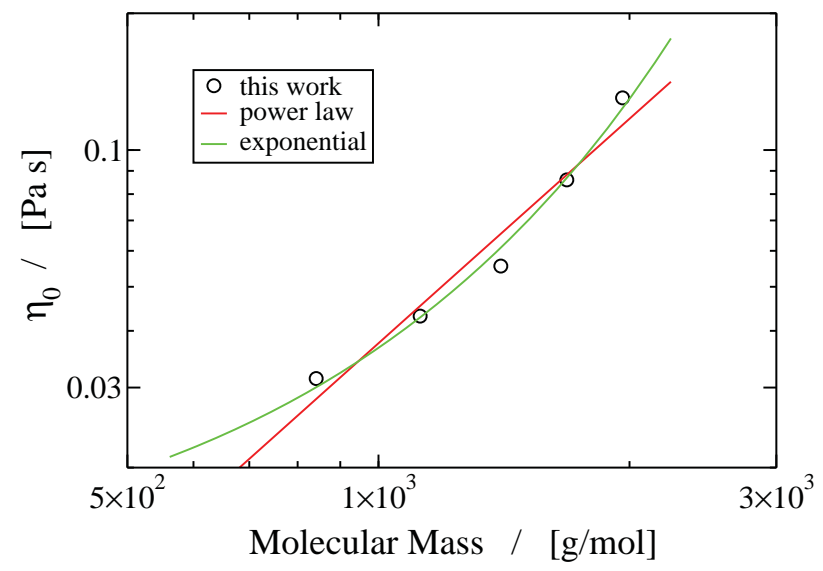

Figure 3.12: Zero shear viscosities for five melts of three-armed stars polymers, $\mathrm{B}\left(\mathrm{B}_{3}\right)_{3}$ through $\mathrm{B}\left(\mathrm{B}_{7}\right)_{3}$, plotted against the molecular mass. An exponential function (green line) fits the data better than a power law (red line).

\subsubsection{Rouse modes}

To get a better appreciation of the role of entanglements in the stress relaxation modulus of the star polymers, we have calculated the $G(t)$ as expected for star polymers obeying Rouselike dynamics with relaxation times extracted from the simulations. In Rouse theory, the blob coordinates $\mathbf{R}_{i}$ are mapped onto Rouse coordinates $\mathbf{X}_{k}$ by a linear transformation that decouples the motions of the Rouse coordinates. [34,63] The relaxations of these modes are well described by stretched exponentials, [45, 46, 63]

$$
C_{k}(t)=\frac{\left\langle\mathbf{X}_{k}(t) \cdot \mathbf{X}_{k}(0)\right\rangle}{\left\langle\mathbf{X}_{k}^{2}\right\rangle}=e^{-\left(t / \tau_{k}^{*}\right)^{\beta_{k}}},
$$

with $\tau_{k}^{*}$ the relaxation time (deviant from the conventional Rouse time obtained for $\beta_{k}=1$ ) and $\beta_{k}$ the stretching exponent. For a collection of $N_{c}$ uncorrelated polymers, each on average adhering to the above relaxation process, it is shown in Appendix 3.4 that

$$
G(t) \approx \frac{N_{c} k_{B} T}{V} \sum_{k=1}^{N-1} e^{-2\left(t / \tau_{k}^{*}\right)^{\beta_{k}}},
$$

with $N$ the number of beads per chain. Hence, the $N-1$ relaxation curves $C_{k}(t)$ were calculated from the blob coordinates stored during the simulations of the stars, and both $\tau_{k}^{*}$ and 


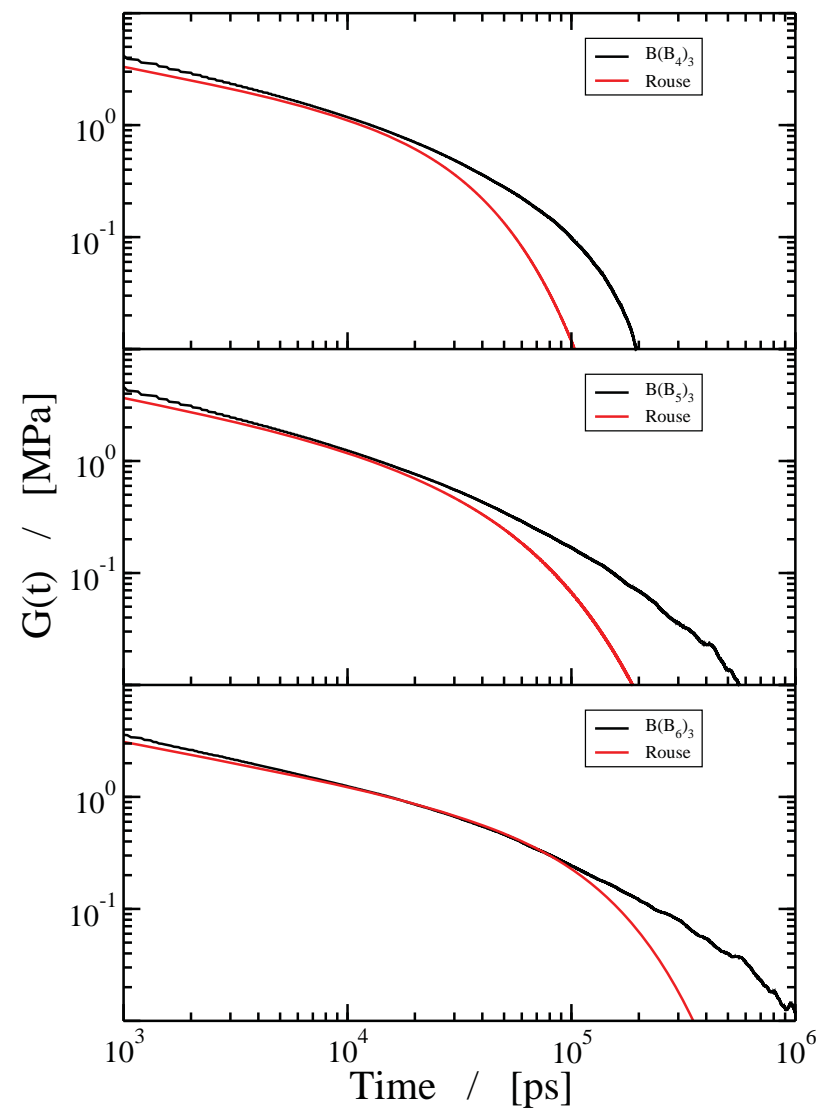

Figure 3.13: Stress relation moduli of the simulated star polymers (black) compared against the theoretical predicted curves for Rouse-like star polymers with the same transient relaxation dynamics.

$\beta_{k}$ were extracted by fitting the initial decay by a stretched exponential. The resulting relaxation times show the same degeneracy pattern as the theoretical Rouse times derived in the appendix; the stretching exponents mostly lie between 0.7 and 1 . Inserting the fitted values into Eq. (3.14) produces the red curves in Fig. 3.13, plotted alongside the actual stress relaxation functions in black. The pairs of curves show a satisfactory agreement for short times, certainly in view of the assumptions made in deriving Eq. (3.14), while at large times the 


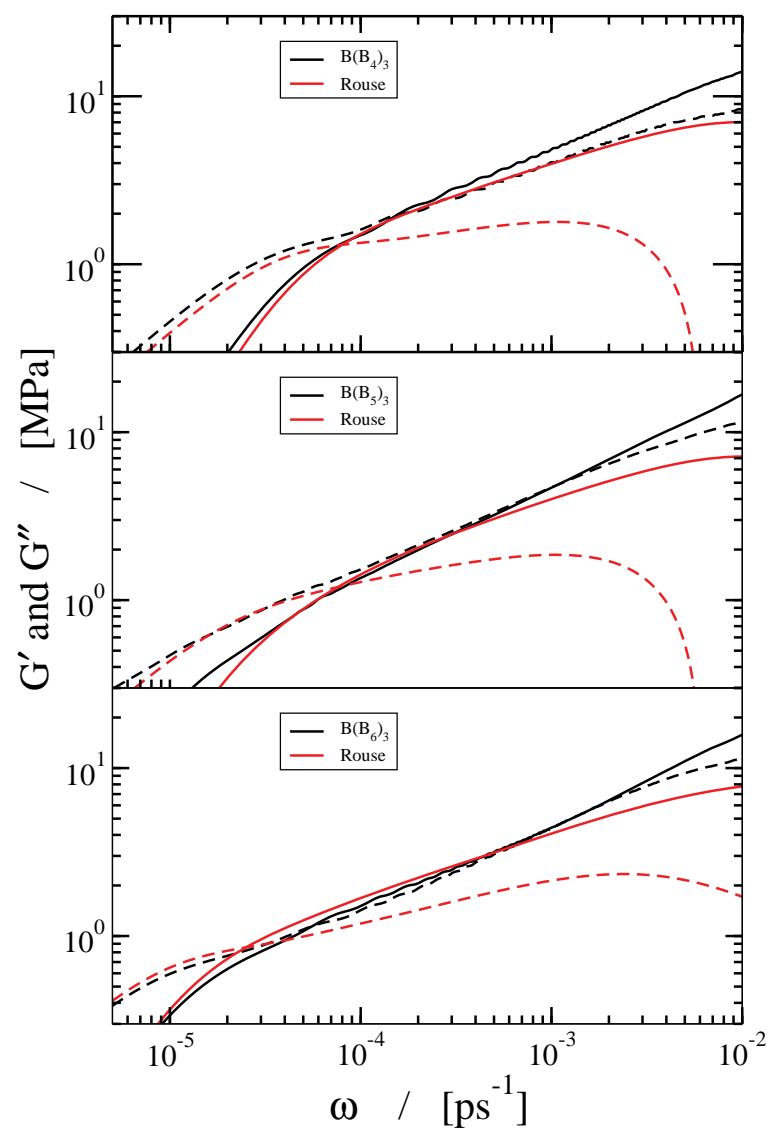

Figure 3.14: Three arm star $G$ ' and $G$ ” compared with stretch Rouse modulus.

slowing down of the relaxation dynamics due to the entanglements becomes visible again.

The storage and loss moduli following the Rouse model with stretched exponentials are shown in Fig. 3.14. In agreement with the direct analysis of the star polymer simulations, the wedge between the storage and loss moduli decreases with increasing arm length. The angle of the wedge, however, is considerably wider than in the direct analysis. 


\subsection{Conclusions}

The diffusive and rheological properties of linear and three-armed polyethylene polymers of various lengths have been studied by computer simulations using the highly coarse grained TWENTANGLEMENT technique. The scaling behaviour of the zero shear viscosity indicates that the linear polymers are in the transition region between Rouse and reptation dynamics. Both the diffusion coefficient and the viscosity are in quantitative agreement with experimental data, while most other coarse grain simulation approaches for polymers are limited to quantitative agreement for either $D$ or $\eta$. Comparing the simulated symmetric star polymers with linear polymers, matching in mass or end-to-end span, highlights the impact of the architecture on the dynamical properties of the melt: Stars are seen to diffuse slower than both matching linear polymers, while the stress relaxation curve lies between those of the matching linear polymers. The viscosity of the linear polymers follows a power law in the molecular mass, the star polymers show exponential scaling in stead. A qualitative agreement with experiments is observed for the wedge between the storage and loss moduli, with linear polymers yielding a larger wedge than star polymers. The quantitative validation of the star simulations has to wait till experimental data on three-armed stars with short arms become available.

\section{APPENDIX : STRESS RELAXATION MODULUS}

The objective in this appendix is to derive Eq. (3.14) for the stress relaxation modulus in a melt of polymers with Rouse-like dynamics. The stress tensor of a melt in a specific configuration, neglecting the kinetic contributions, is equal to

$$
\boldsymbol{\sigma}=-\frac{1}{V} \sum_{a=1}^{N_{c}} \sum_{i=1}^{N} \mathbf{R}_{a i} \mathbf{F}_{a i},
$$

with $V$ the volume of the simulation box, $\mathbf{R}_{a i}$ the position vector of particle $i$ of polymer $a$, $\mathbf{F}_{a i}$ the total force on this particle, $N_{c}$ the number of chains and $N$ the number of blobs per chain. The total stress can be expressed as

$$
\boldsymbol{\sigma}=\sum_{a=1}^{N_{c}} \boldsymbol{\sigma}_{a}^{\prime}
$$


with the stress tensor of polymer $a$ given by

$$
\boldsymbol{\sigma}_{a}^{\prime}=-\frac{1}{V} \sum_{i=1}^{N} \mathbf{R}_{a i} \mathbf{F}_{a i}
$$

In a mean field approximation, the stress tensors of two polymers $a$ and $b$ are uncorrelated,

$$
\left\langle\boldsymbol{\sigma}_{a}^{\prime}(t) \boldsymbol{\sigma}_{b}^{\prime}(0)\right\rangle=\delta_{a b}\left\langle\boldsymbol{\sigma}^{\prime}(t) \boldsymbol{\sigma}^{\prime}(0)\right\rangle,
$$

where for notational convenience we have dropped the polymer label on the right hand side. Dropping this label also from the polymeric stress yields

$$
\boldsymbol{\sigma}^{\prime}=-\frac{1}{V} \sum_{i=1}^{N} \mathbf{R}_{i} \mathbf{F}_{i}
$$

with the forces $\mathbf{F}_{i}$ still including interactions between polymers. We now make the assumption that the inter-molecular forces can be taken into account by assuming effective intramolecular forces, which is supported by the subsequent results. Since the sum over all internal forces is zero, this renders the polymeric stress independent of the polymer's position within the box. For beads connected by springs, the forces then take the form

$$
F=-\xi w T R
$$

where $\xi$ is the friction coefficient and $w$ the elementary Rouse rate. The positions $\mathbf{R}_{i}$ have been collected in the long vector $R$, with the $N$ entries in this vector representing coordinate vectors rather than Cartesian coordinates; the long force vector is constructed likewise. The matrix $T$ describes the structure of the polymer: the diagonal elements count the number of connections of a bead, the off-diagonal elements are -1 for connected bead pairs and zero otherwise; an example hereof for a three-armed star can be found in Ref.(33). Combining Eq. (A.6) with Eq. (A.5) yields the polymeric stress

$$
\boldsymbol{\sigma}^{\prime}=\frac{\xi w}{V} R^{T} T R
$$

At this point it is convenient to make the transition from correlated blob coordinates to uncoupled Rouse modes. The long vector $X$ of Rouse vectors is obtained by a linear combination of the position vectors, $X=S R$, where $S$ is the orthogonal matrix that diagonalizes $T$ into the matrix $\Lambda$ of eigenvalues $\left\{\lambda_{1}, \ldots, \lambda_{N_{c}}\right\}$. As a result of the symmetry in the star architecture, 
and hence $T$, several of these eigenvalues are degenerate. Expressing the stress in terms of the Rouse vectors gives

$$
\boldsymbol{\sigma}^{\prime}=\frac{\xi w}{V} X^{T} \Lambda X=\frac{\xi w}{V} \sum_{k=1}^{N-1} \lambda_{k} \mathbf{X}_{k} \mathbf{X}_{k} .
$$

Applying the equipartition theorem to the potential energy expression matching Eq. (A.6) yields

$$
\left\langle\mathbf{X}_{k} \cdot \mathbf{X}_{k}\right\rangle=\frac{3 k_{B} T}{\xi w \lambda_{k}}
$$

and therefore

$$
\boldsymbol{\sigma}^{\prime}=\frac{3 k_{B} T}{V} \sum_{k=1}^{N-1} \frac{\mathbf{X}_{k} \mathbf{X}_{k}}{\left\langle\mathbf{X}_{k} \cdot \mathbf{X}_{k}\right\rangle} .
$$

The diagonal elements of the force contribution to the total stress produce the average

$$
\left\langle\sigma_{\alpha \alpha}\right\rangle=N_{c}\left\langle\sigma_{\alpha \alpha}^{\prime}\right\rangle=\frac{N_{c}(N-1)}{V} k_{B} T .
$$

This large term - note that the blob density in the simulations is nearly two orders higher than the molecule density in a gas at ambient conditions - cancels almost completely against the kinetic contribution to the stress.

Turning now to the off-diagonal elements of the stress, the shear relaxation modulus is given by

$$
\begin{aligned}
G(t) & =\frac{V}{k_{B} T}\left\langle\sigma_{\alpha \beta}(t) \sigma_{\alpha \beta}(0)\right\rangle \\
& =N_{c} \frac{V}{k_{B} T}\left\langle\sigma_{\alpha \beta}^{\prime}(t) \sigma_{\alpha \beta}^{\prime}(0)\right\rangle,
\end{aligned}
$$

where Eq. (A.4) was used in the last step. Inserting Eq. (A.10) yields a double summation, with indices $k$ and $k^{\prime}$, over terms of the form

$$
\begin{aligned}
\left\langle\mathbf{X}_{k \alpha}(t) \mathbf{X}_{k \beta}(t)\right. & \left.\mathbf{X}_{k^{\prime} \alpha}(0) \mathbf{X}_{k^{\prime} \beta}(0)\right\rangle \\
& =\left\langle\mathbf{X}_{k \alpha}(t) \mathbf{X}_{k \beta}(t)\right\rangle\left\langle\mathbf{X}_{k^{\prime} \alpha}(0) \mathbf{X}_{k^{\prime} \beta}(0)\right\rangle \\
& +\left\langle\mathbf{X}_{k \alpha}(t) \mathbf{X}_{k^{\prime} \alpha}(0)\right\rangle\left\langle\mathbf{X}_{k \beta}(t) \mathbf{X}_{k^{\prime} \beta}(0)\right\rangle \\
& +\left\langle\mathbf{X}_{k \alpha}(t) \mathbf{X}_{k^{\prime} \beta}(0)\right\rangle\left\langle\mathbf{X}_{k \beta}(t) \mathbf{X}_{k^{\prime} \alpha}(0)\right\rangle \\
& =\delta_{k k^{\prime}}\left\langle\mathbf{X}_{k \alpha}(t) \mathbf{X}_{k \alpha}(0)\right\rangle\left\langle\mathbf{X}_{k \beta}(t) \mathbf{X}_{k \beta}(0)\right\rangle \\
& =\frac{1}{9} \delta_{k k^{\prime}}\left\langle\mathbf{X}_{k}(t) \cdot \mathbf{X}_{k}(0)\right\rangle^{2},
\end{aligned}
$$


where the first step follows from the Isserlis-Wick theorem, the second step is based on the absence of correlations between orthogonal directions, and the last step employs the equivalence of all three Cartesian directions. The Kronecker delta on the last line reduces the double summation in $G(t)$ into a single summation,

$$
G(t)=N_{c} \frac{k_{B} T}{V} \sum_{k=1}^{N-1} \frac{\left\langle\mathbf{X}_{k}(t) \cdot \mathbf{X}_{k}(0)\right\rangle^{2}}{\left\langle\mathbf{X}_{k} \cdot \mathbf{X}_{k}\right\rangle^{2}}
$$

For the ideal Rouse polymer, the internal force in Eq. (A.6) gives rise to exponentially decaying auto-correlation functions for $\mathbf{X}_{k}$, with relaxation times $\left(w \lambda_{k}\right)^{-1}$. The motion of an actual polymer is affected by the surrounding polymers, resuling in the transient decay of the auto-correlation being better described by the stretched exponentials of Eq. (3.13). Inserting these stretched exponentials into Eq. (A.15) produces Eq. (3.14). 



\section{Dynamics of Asymmetric Star Polymers under Coarse Grain Simulations}

In this chapter we study asymmetric star polymers by coarse grain simulations. Each polymer is represented by a branched string of soft blobs, and uncrossability constraints are added to prevent chain crossings. Two types of asymmetric three-armed stars are considered, with varying lengths for the differing arm to asses their impact on the viscoelastic properties of the melt. Changes in the relaxation dynamics of the melts are already discernable for short arms, well below the entanglement mass. The analysis of the dynamical properties of the larger stars, with arm lengths approaching the entanglement length, indicate that infrequent arm retraction events are the main mechanism slowing down the relaxation dynamics of the melt.

\subsection{Introduction}

It is well known that the dynamic and viscoelastic properties of polymer melts depend on the architecture of the polymer [7, 14,27,31,34-36,61,87,102,108-110]. Experimental studies of long linear polymers show that the diffusion coefficient and viscosity scale as power laws with the polymer mass [27-29]. This behaviour is explained by the reptation model of de Gennes, Doi and Edwards: the polymers are diffusing along the confining tube formed by their neighbours $[35,36]$. A long star-like polymer will have each of its arms confined to a separate tube, making it impossible for the star to move by the reptation mechanism. In marked difference to the linear polymers, stars are observed to follow exponential scaling 
laws in the experiments $[6,14,61,87,111]$. This behaviour is explained by the 'arm retraction' theory of de Gennes: the branching point of a star can only move when the constraints on an arm are released by full contraction along its primitive path [80]. The calculation of the retraction rate, in terms of Kramers' thermally activated escape rate from a potential well, as presented by Doi and Kuzuu [81] and subsequently extended by Pearson and Helfand [82], yields the exponential dependence on the arm length. Viscoelastic properties derived using these models are in good agreement with experiments [14, 31, 61]. These concepts have also been applied to polymers with complex multiply branched architectures by Milner and McLeish and the Larson group [18, 88, 91, 112, 113].

Computer simulations of polymer dynamics at the level of atoms or chain segments are being used by a number of groups to probe the above concepts. A complication inherent to these studies is the extremely slow dynamics of highly entangled linear and star polymers; this makes the simulations very computer intensive, even when using coarse-grained models. Reptation-like behaviour has been observed in a number of simulations $[7,11,66,74,92]$. A first, somewhat inconclusive indication of hopping motion of the branch point was observed by [96]. Masubuchi et al. noted that suppression of the branch point motion resulted in unreasonably slow relaxation dynamics [99]. For symmetric stars, motion of the branch point along the tube of one of the arms has been reported by Bačová et al. for a selected star with fixed end points in a melt of free stars [97]. So, while a direct correlation between branch point motion and arm configurations has not yet been established, these simulations, as well as experiments with tracers attached the branch point, support the theoretical frame work based on branch point motion.

In this chapter, we set forth to explore the dynamics of melts of asymmetric stars. We will investigate how the rheology of the stars changes when going from asymmetric to symmetric stars, and discuss our results in the light of arm retraction. Our polymers are simulated by the TWENTANGLEMENT approach, as extended and applied in the previous chapters. After a brief explanation of the simulation model and analysis techniques in Section 4.2, the simulation results on two classes of asymmetric stars are presented and discussed in Section 4.3. conclusion is made in Section 4.4. 

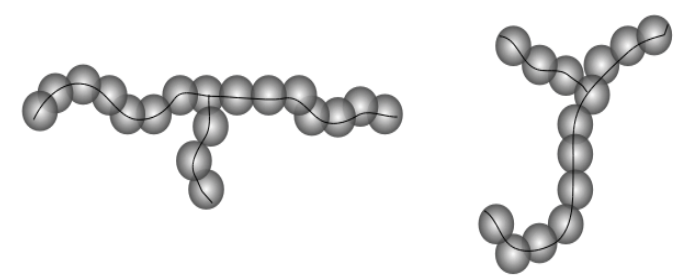

Figure 4.1: Schematic diagrams of two 3-arm asymmetric star. On the left the 'T-shaped' $\mathrm{B}\left(\mathrm{B}_{3}\right)\left(\mathrm{B}_{7}\right)_{2}$ with one short and two long arms, and on the right the ' $Y$ '-shaped $\mathrm{B}\left(\mathrm{B}_{7}\right)\left(\mathrm{B}_{3}\right)_{2}$ with one long and two short arms.

\subsection{Model and methods}

\subsubsection{Simulation model}

In this chapter we will apply the coarse grained TWENTANGLEMENT model, as already presented in Chapters 2 and 3, to simulate asymmetric stars of polyethylene. Briefly, each coarse grained particle or 'blob' represents a polymer fragment of 20 backbone carbon atoms plus attached hydrogen atoms. The asymmetric three-armed stars follow the generic formula $\mathrm{B}\left(\mathrm{B}_{m}\right)\left(\mathrm{B}_{n}\right)_{2}$, where the first symbol B stands for the branching point, connected to one arm of $m$ blobs and two arms of $n$ blobs each. Henceforth, stars with one short ( $m=3,5$ or 7 ) and two long $(n=7)$ arms will be referred to as 'T'-shaped, while stars with one long $(m=3$, 5 or 7$)$ and two short $(n=3)$ arms are called ' $Y$ '-shaped, as illustrated by the examples in Fig. 4.1.

The coarse-graining procedure gives rise to a stochastic equation of motion for the blobs. The change in the position $\mathbf{R}_{i}$ of particle $i$ over a time-step $d t$ is calculated using a first order Langevin equation,

$$
d \mathbf{R}_{i}=-\frac{1}{\xi} \mathbf{F}_{i}^{C} d t+\delta \mathbf{R}_{i} .
$$

Here, $\xi$ denotes the friction coefficient of the blob, and the conservative force is derived from a potential of mean force,

$$
\mathbf{F}_{i}^{C}=-\nabla_{i} \Phi,
$$


to be discussed below. In the second term on the right of Eq. (4.1), the random Markovian displacements have zero mean $\left\langle\delta \mathbf{R}_{i}(\tau)\right\rangle=0$ and are related to the friction in the first term by the fluctuation-dissipation theorem,

$$
\left\langle\delta \mathbf{R}_{i}(\tau) \delta \mathbf{R}_{j}\left(\tau^{\prime}\right)\right\rangle=\frac{2 k_{B} T}{\xi} 1 \delta_{i j} \delta_{\tau, \tau^{\prime}} d t,
$$

where $\mathbf{1}$ is the unit matrix, $k_{B}$ the Boltzmann constant, $T$ the temperature and where the two Kronecker deltas have the value of one if their two indices (particle numbers and step numbers, respectively) are equal and the value of zero if their two indices are unequal.

The employed potential of mean force was derived from the probability distribution $P$ of blob centers of mass in atomistic simulations of a $\mathrm{C}_{120} \mathrm{H}_{242}$ melt, by using $\Phi=-k_{B} T \ln P\left(\mathbf{R}^{n}\right)$. For convenience, this potential was approximated as a sum of contributions, with $\phi^{\text {bond }}\left(l_{i, i+1}\right)$ the attraction between two bonded blobs $i$ and $i+1$ at a separation $l_{i, i+1}=\left|\mathbf{R}_{i+1}-\mathbf{R}_{i}\right|$, with $\phi^{\text {ang }}(\theta)$ the bending potential relating to the angle $\theta$ formed by three consecutive blobs, $\phi^{\mathrm{nb}}\left(R_{i j}\right)$ the non-bonded interaction between two blobs $i$ and $j$, and $\phi^{\mathrm{rep}}\left(R_{i, i+1}\right)$ the repulsion between two bonded blobs $i$ and $i+1$. We refer the reader to earlier work for a detailed discussion of the potential $[44,114]$.

Because each blob represents a flexible collection of bonded atoms, the non-bonded repulsive interactions between blobs are much softer than those between atoms. The repulsions are, in fact, too weak to inhibit crossings of the coarse grained polymers and thus a key property determining the dynamics of polymer melts is lost upon coarse-graining. To prevent these unrealistic crossing events, Padding and Briels developed the TWENTANGLEMENT algorithm that uses elastic forces to contain and reverse bond crossings [44]. In short: an 'entanglement point' $\mathbf{X}$ is introduced at the point where the bond between blobs $i$ and $i+1$ first crosses another bond. The binding energies $\phi^{\text {bond }}$ of these bonds are henceforth calculated using the contour length $L_{i, i+1}$ of the path from blob to blob via the entanglement point. The path between two consecutive blobs can collect multiple entanglement points by 'crossing' other paths, resulting in twisting paths of contour length

$$
L_{i, i+1}=\left|\mathbf{R}_{i}-\mathbf{X}_{1}^{i, i+1}\right|+\left|\mathbf{X}_{1}^{i, i+1}-\mathbf{X}_{2}^{i, i+1}\right|+\ldots+\left|\mathbf{X}_{p}^{i, i+1}-\mathbf{R}_{i+1}\right|,
$$

running from one bead to the next via a sequence of $p$ entanglement points. Beads may slide along an entanglement point, thereby causing the entanglement point to slip from one bond to the next. An entanglement point survives until the two involved bond paths disentangle, 
at which occasion the entanglement point is annihilated. The positions of the entanglement points are recalculated every time step by minimizing the elastic energy stored in the bonds, subject to fixed blob positions. The forces exerted by the entanglements on the blobs, by means of the bond energy $\phi^{\text {bond }}\left(L_{i, i+1}\right)$, are included in the equation of motion of the blobs. The interested reader is referred to Padding and Briels [44] for further details on the TWENTANGLEMENT algorithm. We have recently extended this algorithm to simulate branched polymers. The modifications, an extensive set of rules governing the slip of an entanglement point over the branching point and a stiffening of the bending potential at the branch point, are discussed and validated by simulations in Ref. [46]. An application of this algorithm to symmetric stars is presented in chapter 3, where it is observed - in agreement with theory and experiments $[36,61,81,82]$ - that the viscosity of a melt of stars scales exponentially with the mass of the stars, in contrast with the linear scaling for linear polymers.

All simulations were performed in cubic boxes with periodic boundary conditions. Typical boxes contained 80 to 100 polymers, at a density of $\rho=0.761 \mathrm{~g} / \mathrm{cm}^{3}$. The friction coefficient was set at $\xi=32.0 \mathrm{ps}^{-1}$, in combination with a time step $d t=0.4$ ps. A temperature of $T=450 \mathrm{~K}$ ensured that the systems are in the melt phase. In addition to the T-shaped $\mathrm{B}\left(\mathrm{B}_{m}\right)\left(\mathrm{B}_{7}\right)_{2}$ and the $\mathrm{Y}$-shaped $\mathrm{B}\left(\mathrm{B}_{m}\right)\left(\mathrm{B}_{3}\right)_{2}$ polymers, with $m=3,5$ and 7 , we also simulated linear polymers $\mathrm{B}_{n}$, with $n=7,9,11,13,16,19$ and 22 , for comparison purposes.

\subsubsection{Analysis methods}

The dynamical properties of the branched polymers are analysed in several distinct ways. Diffusion coefficients are extracted from the mean square displacement (MSD)

$$
D(t)=\frac{1}{6 t}\left\langle\left|\mathbf{R}_{i}^{\mathrm{com}}(t)-\mathbf{R}_{i}^{\mathrm{com}}(0)\right|^{2}\right\rangle,
$$

with $\mathbf{R}^{\mathrm{com}}(t)$ the center of mass position of polymer $i$ at time $t$, and the pointed brackets indicating an average over all times and polymers. Arm dynamics is analyzed by the autocorrelation

$$
f_{b e}(t)=\left\langle\delta L_{i}^{e b}(t) \delta L_{i}^{e b}(0)\right\rangle
$$

of arm length fluctuations $\delta L_{i}^{e b}(t)=\delta L_{i}^{e b}(t)-\left\langle L_{i}^{e b}\right\rangle$, with the instantaneous length $L_{i}^{e b}(t)$ of an arm measured as the direct distance from the branch point $\mathbf{R}_{i}^{b}$ to the end point of the arm $\mathbf{R}_{i}^{e}$. 
The central rheological property in this study is the stress relaxation function, which is calculated from the thermal fluctuations of the stress tensor,

$$
G(t)=\frac{V}{k_{B} T}\left\langle\sigma_{\alpha \beta}(t) \sigma_{\alpha \beta}(0)\right\rangle .
$$

Here $V$ is the total volume of the simulation box, $\alpha$ and $\beta$ are two distinct Cartesian directions, and the microscopic stress tensor is given by

$$
\sigma_{\alpha \beta}=-\frac{1}{V} \sum_{i, j}\left(y_{i, \beta}-y_{j, \beta}\right) F_{i j, \alpha},
$$

where $i$ and $j$ run over all entities - blobs and entanglement points - in the box, $y_{i \alpha}$ denotes the $\alpha^{\text {th }}$ coordinate of the $i^{\text {th }}$ entity, and $F_{i j, \alpha}$ is the $\alpha$ component of the force on entity $i$ exerted by entity $j$. Since the signal to noise ratio of the stress relaxation function strongly decreases with $t$, the late-time plots have been smoothed by block-averaging,

$$
\bar{G}(t)=\int_{-\Delta}^{\Delta} G(t+\tau) w(\tau) d \tau .
$$

In view of the logarithmic time axis in these figures, the normalized weight function was selected as $w(\tau) d \tau \propto d \ln (\tau)$ and the block width $\Delta$ increased exponentially with $t$. To facilitate the comparison with experimental data, we also calculated the Fourier transforms of the stress relaxation function,

$$
\begin{aligned}
G^{\prime}(\omega) & =\omega \int_{0}^{\infty} \sin (\omega t) G(t) d t, \\
G^{\prime \prime}(\omega) & =\omega \int_{0}^{\infty} \cos (\omega t) G(t) d t,
\end{aligned}
$$

which are known as the storage and loss moduli, respectively. These integrals were calculated using the raw data $G(t)$ up to the first appearance of negative values; for larger times, $G(t)$ was approximated by a single decaying exponential fitted to the tail region of the block-averaged $\bar{G}(t)$.

\subsection{Results and Discussion}

\subsubsection{T-shaped polymers}

In the simulated T-shaped polymers, a side chain of 3, 5 or 7 beads is attached to the central bead of a linear backbone of 15 beads, see Fig. 4.1. The former two side chains generate 


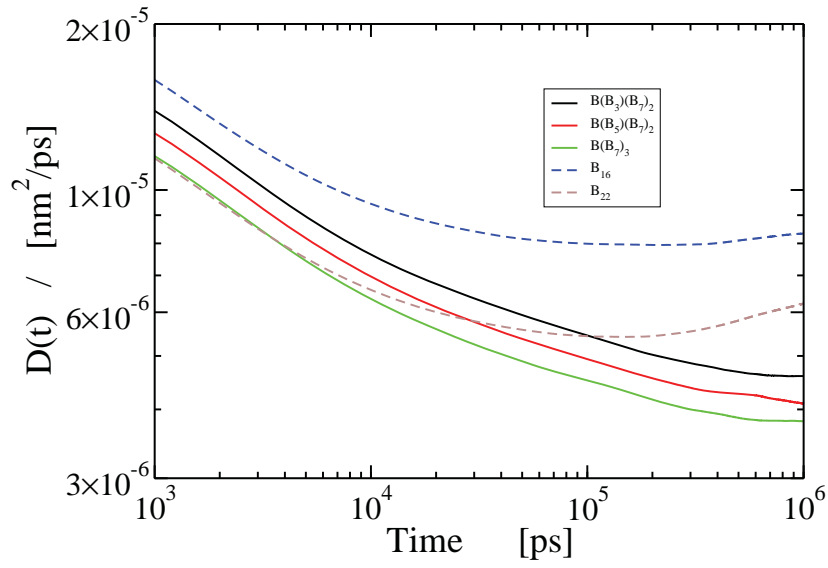

Figure 4.2: Time dependent diffusion coefficients of three T-shaped polymers (solid lines) and two linear polymers (dashed lines). The shortest linear polymer is of comparable length to the back bone of the T-shaped polymers, the longest linear polymer has the same mass as the largest T-shaped polymer, i.e. the symmetric star $\mathrm{B}\left(\mathrm{B}_{7}\right)_{3}$.

asymmetric stars, the latter side chain creates a symmetric star. The time dependent diffusion coefficients of these three stars are plotted as solid lines in Fig. 4.2, where they are seen to steadily decline over time. As expected, the diffusion coefficient decreases as the side arm becomes longer. Comparing the T-shaped polymers with linear polymers, included as dashed lines in Fig. 4.2, highlights the impact of the side arm. The three-bead side arm of $\mathrm{B}\left(\mathrm{B}_{3}\right)\left(\mathrm{B}_{7}\right)_{2}$ has roughly halved the polymer's diffusion coefficient relative to a melt of the backbones alone, as represented by the linear $\mathrm{B}_{16}$ polymer. Whereas these polymers are both expected to reptate in a single tube, shown as Fig. 4.3(a) and (b) - the short side chain is estimated at less than halve the entanglement length - the enhanced friction due to the side arm proves an impediment for the diffusive motion along the tube. With increasing length of the short arm, the diffusion of the T-shaped stars will gradually change from being dominated by reptation along the backbone tube, to being dominated by branch point hopping. Fig. 4.2 shows that the diffusion coefficient drops substantially with increasing length of the short arm.

Figure 4.4 shows the relaxation dynamics of the short and long arms of the T-shaped 


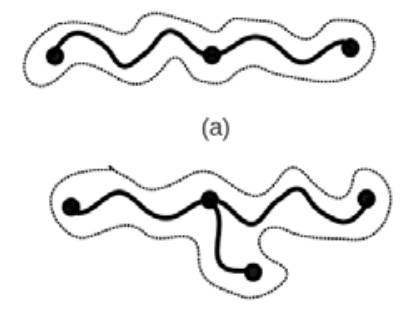

(b)

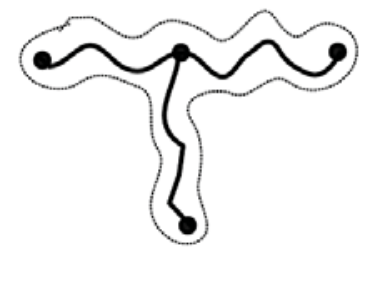

(c)

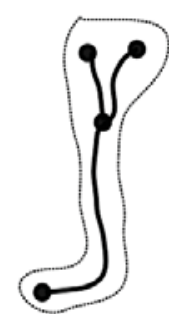

(d)

Figure 4.3: Cartoons illustrating the shape of the tube (dashed lines) surrounding polymers (thick black lines, with dots highlighting end points and branching points) of various topologies: a) linear polymer, b) T-shaped polymer with two long and one short arm, c) a star polymer with three long arms, and d) Y-shaped polymer with one long and two short arms.

polymers. The rapid transient relaxation of the arm length is attributed to length fluctuations within a constant arm configuration (short arms) or within a fixed confining tube (long arms), i.e. contour length fluctuations. The subsequent slow decay of the arm length is attributed to transitions of the arm configuration (short arms) or of the confining tube (long arms). Evidently, the long-time relaxation times of the arms increase with increasing arm length, but they also depend on the length of the short arms, as is clear from the relaxation of the length of the 7-blob arms in the three different T-shaped polymers. This effect results both from mechanical coupling within the molecule, since the arms are covalently bond at the branch point, and from the dynamics of the arms of adjacent molecules in the melt, as these arms contribute to the confinement of the arms of the primary polymer. For the symmetric star with arms of 7 blobs, the relaxation time $\tau_{7}$ of the arm length is about $0.8 \mu \mathrm{s}$. The distinction of time scales exhibited in Fig. 4.4 can also be discerned in Fig. 4.5, which shows that the typical time evolution of the length of an arm, in melts of $\mathrm{B}\left(\mathrm{B}_{7}\right)_{3}$, is a superposition of fast and slow dynamics. The time evolution shown here suggests that the relaxation of the arm length is largely determined by transitions between stable states, which live for a few tenths of microseconds in rough agreement with $\tau_{7}=0.8 \mu$ s, and indicative of changes of the tube of the arm. Complete retraction of the arms, which is here operationally defined as the arm length being less than $10 \%$ of its average length, occurs about once every $10^{5}$ 


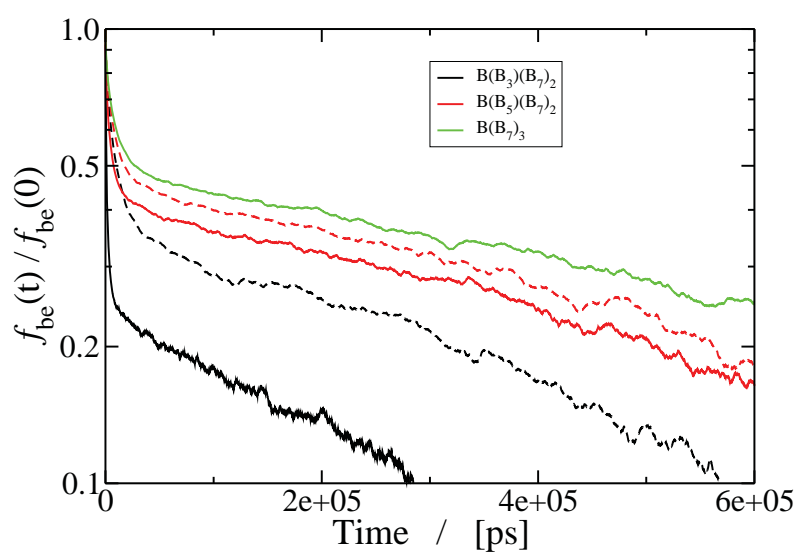

Figure 4.4: Relaxation of the arm length, from branch point to end point, of the short arm (solid lines) and the long arms (dashed lines) of three T-shaped polymers, as calculated by Eq. (4.6).

picoseconds. Attempts to correlate arm retraction to displacements of the branch point have not been successful, the main reason being that the effect is not yet very pronounced with arms barely longer than one entanglement length. In spite of this, it is fair to say that the slow convergence of the diffusion coefficient in Fig. 4.2 and the long tail of the stress relaxation (to be discussed next) are both attributable to the limited number of arm retractions sampled over the course of a $4 \mu$ s simulation. The importance of arm retraction is further supported by the observation that the two comparable linear polymers, whose motion does not rely on arm retraction, have converged to their long-time behaviour - constant $D(t)$ in Fig. 4.2 and exponentially decaying $G(t)$ below - well within $1 \mu \mathrm{s}$, whereas the T-shaped polymers require a considerably longer time.

The stress relaxation function $G(t)$ up to the $20 \mathrm{~ns}$ timescale is shown in Fig. 4.6. The plot reveals clear differences between T-shaped and linear polymers, exceeding the variations within these two sets. At $t=0$ the branched polymers have a higher $G(t)$ than the linear polymers, but by $0.2 \mathrm{~ns}$ this order has inverted. The transient relaxation of the T-shaped polymers is quicker than that of the linear polymers because the former have one additional chain end that can move around and relax the local strain relatively easily in comparison to 


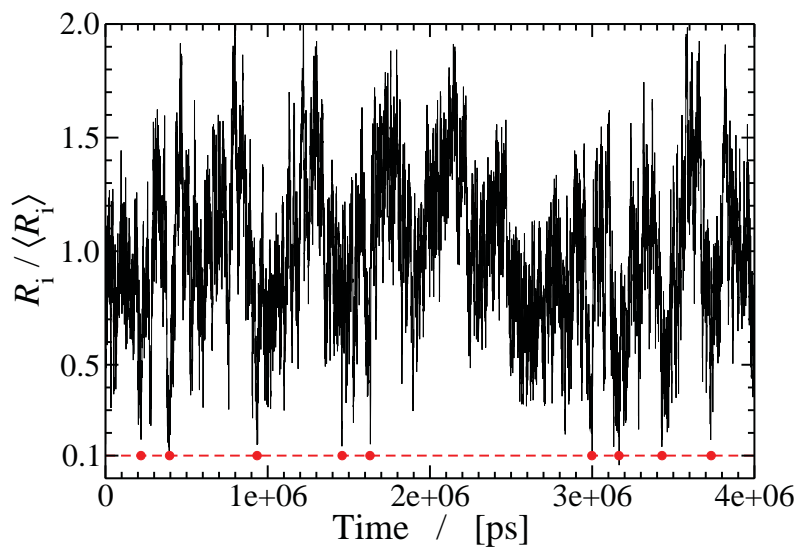

Figure 4.5: Typical time evolution of the length of a long arm in a melt of $\mathrm{B}\left(\mathrm{B}_{7}\right)_{3}$. Arm lengths of less than $10 \%$ of the average arm length, the dashed red line, are exceedingly rare; the plot shows about ten of these 'arm retraction' events, marked by red dots, in a time interval of $4 \mu$ s.

the more slowly relaxing mid-chain segments. In the next time regime, between 1 and $20 \mathrm{~ns}$, a power law behaviour is observed with a power of about -0.5 . The T-shaped polymer with the shortest side arm decays the quickest, and the power slightly increases (becomes less negative) as the side chain becomes longer.

The stress relaxation $\bar{G}(t)$ on the long time scale, from $10 \mathrm{~ns}$ to $1 \mu \mathrm{s}$, is presented in Fig. 4.7, were block-averaging has been invoked to improve the signal-to-noise ratio - an example illustrating the raw data can be found in Fig. (3.1) of this thesis. The near power law decay of $G(t)$ for the linear polymers, as observed in Fig. 4.6 for times exceeding $\sim 1 \mathrm{~ns}$, is seen to gradually turn into a faster exponential decay with increasing time. For the T-shaped polymers, especially those with the longer side chains, this transition is weaker and/or shifted to larger times. At around $0.4 \mu$ s the stress relaxation curves of $\mathrm{B}\left(\mathrm{B}_{5}\right)\left(\mathrm{B}_{7}\right)_{2}$ and $\mathrm{B}\left(\mathrm{B}_{7}\right)_{3}$ are seen to re-cross the stress relaxation curve of the linear polymer with a comparably long backbone, i.e. $\mathrm{B}_{16}$. No re-crossing is observed for the T-shaped polymer with the shortest side chain, however, which continues to relax at approximately the same rate as this linear polymer. The presence in the $\mathrm{B}\left(\mathrm{B}_{3}\right)\left(\mathrm{B}_{7}\right)_{2}$ melt of short, non-entangled and hence relatively agile 


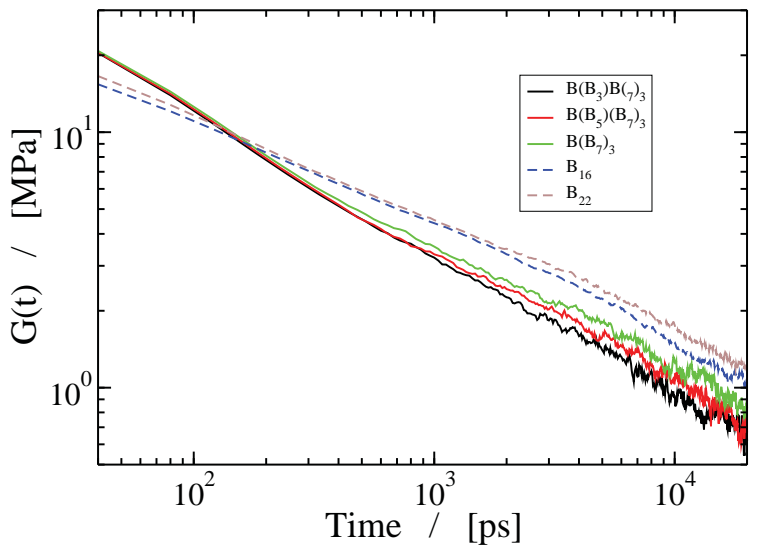

Figure 4.6: Short time stress relaxation $G(t)$ in melts of T-shaped polymers (solid lines) and melts of linear polymers (dashed lines), showing the raw data sampled at intervals of $40 \mathrm{ps.}$

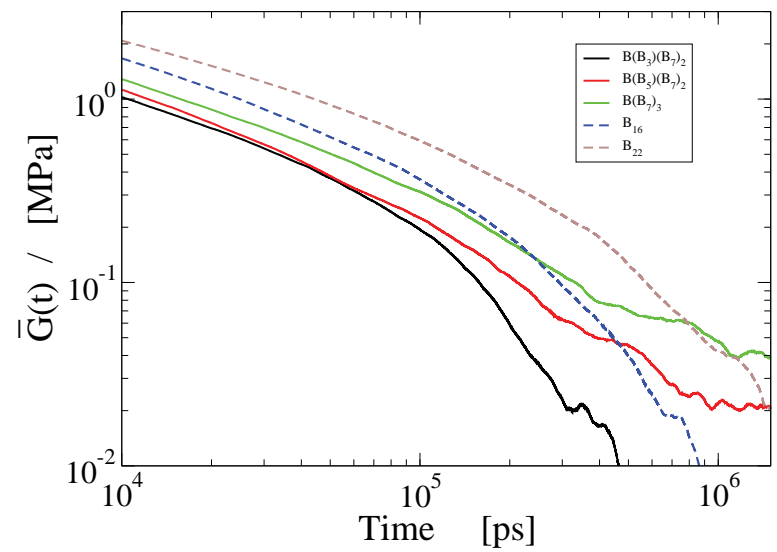

Figure 4.7: Stress relaxation functions $\bar{G}(t)$ for three T-shaped polymers (solid lines), and two linear polymers (dashed lines). To suppress the strong noise, which is already visible on the right hand side of Fig. 4.6 and will gain in relative size as $G(t)$ continues to decay with increasing time, the raw data sampled at 40 ps intervals have been block averaged using the procedure of Eq. (4.9). 


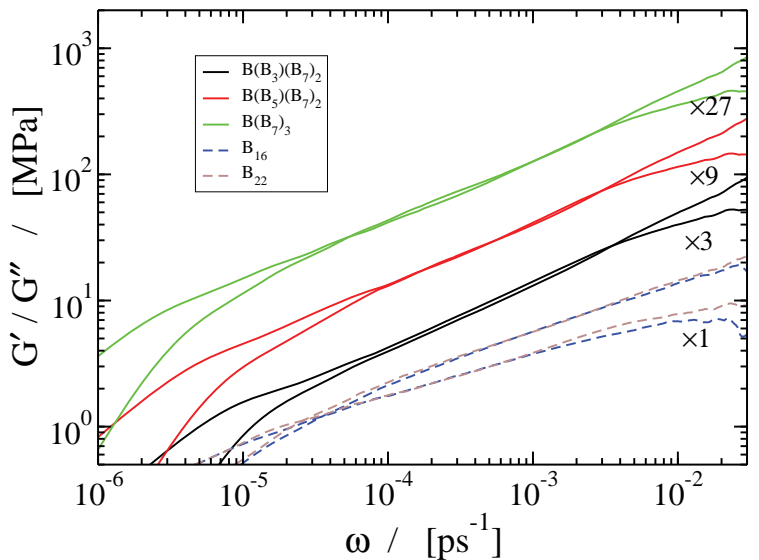

Figure 4.8: Storage and loss moduli of three T-shapes polymers (solid lines) and two linear polymers (dashed lines). For clarity, the curves have been shifted vertically by the multiplication factors indicated next to the lines.

side chains enables relatively fast local relaxations, resulting in even faster decay than with the linear chain of longest span. In spite of this effective 'dilation' of the tube, see Fig. 4.3(b), confining the motion of the backbone, the diffusion coefficients of Fig. 4.2 indicate that the friction incurred by the presence of the short side chain makes this branched polymer diffuse slower than the matching linear polymer. With increasing side chain length, the side chain mobility decreases and also the capability of fast local stress relaxation. When the side chain can form sufficient entanglements to acquire a tube of its own, the dominant diffusion mechanism of the T-branched polymer changes from reptation to arm-retraction followed by branch point hopping. This will slow down the diffusion as well as the stress-relaxation. In view of the shoulder starting to develop in Fig. 4.7, it is tempting to speculate that the 5 and 7 bead side-chains are on the threshold to having their own tubes as shown in Fig. 4.3(c).

The storage and loss moduli calculated from the stress relaxation functions are presented in Fig. 4.8. A striking feature is the coalescence, over approximately two decades in frequency space, of $G^{\prime}$ and $G^{\prime \prime}$ for the T-shaped polymers. We note that the power law behaviour $G(t) \propto t^{-1 / 2}$, as observed in Figs 4.6 and 4.7, gives rise to $G^{\prime}(\omega)=G^{\prime \prime}(\omega) \propto \omega^{1 / 2}$, in agreement with the coalescence and slope in Fig. 4.8. Experimental data for three-armed stars, 


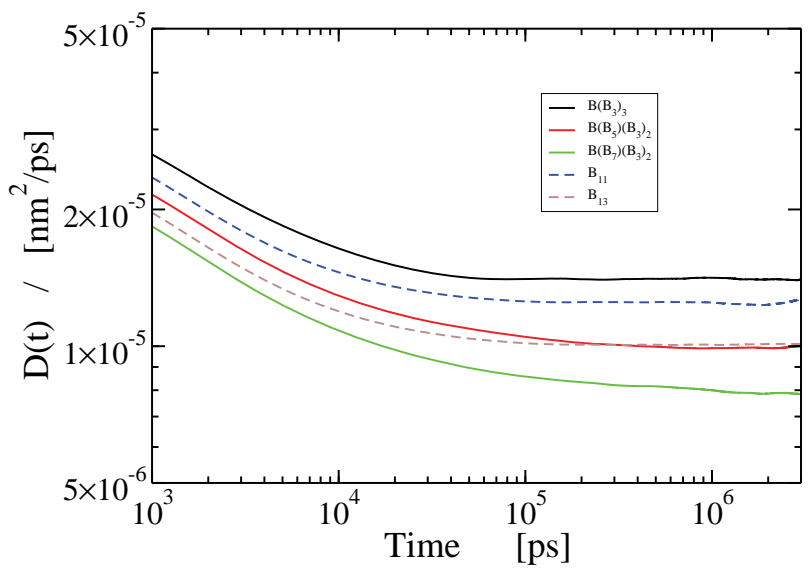

Figure 4.9: Time-dependent diffusion coefficients of three Y-shaped polymers (solid lines), as well as two linear polymers (dashed lines) with equal backbone length or (nearly) equal total weight to the largest $Y$-shaped polymer.

and stars in general $[14,31,61,103]$, show $G^{\prime}$ and $G^{\prime \prime}$ crossing each other at the terminal crossing point. With increasing frequencies, although not running in parallel, the two curves continue with slopes much less disparate than for linear polymers of corresponding masses. We attribute the differences between the simulates and the experimental curves to the large difference in the lengths of the polymers being analyzed.

\subsubsection{Y-shaped polymers}

The simulated Y-shape polymers, see Fig. 4.1, combine two short arms of three blobs each with one longer arm of 3,5 or 7 blobs. Alternatively, these polymers may be viewed as linear chains of 7, 9 or 11 blobs with a side chain of 3 blobs attached to the fourth backbone blob. The smallest of these polymers is a symmetric star, with arms of three beads each. Figure 4.9 shows that the time-dependent diffusion coefficients of these polymers converge to stationary values within about $0.1 \mu \mathrm{s}$. In our earlier work [46], we noted that the diffusion coefficient of the symmetric star $\mathrm{B}\left(\mathrm{B}_{3}\right)_{3}$ was very similar to that of a linear polymer of equal mass, $\mathrm{B}_{10}$. Comparing the largest of the Y-shaped polymers against two linear polymers, of 


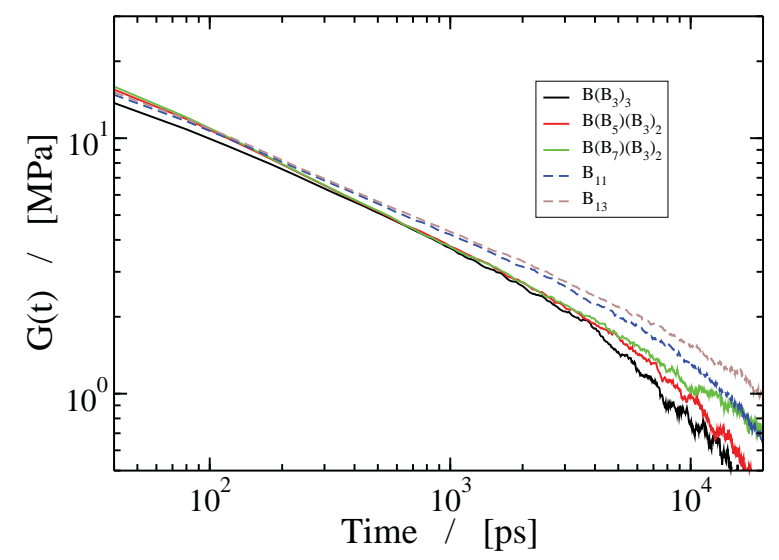

Figure 4.10: Short time stress relaxation in melts of Y-shaped polymers (solid lines) and in melts of linear polymers (dashed lines), showing raw data sampled at intervals of $40 \mathrm{ps}$.

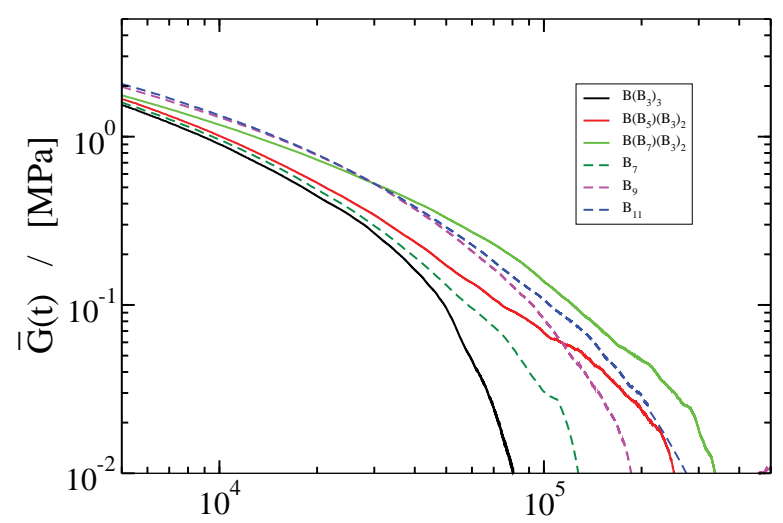

Figure 4.11: Long time stress relaxation in melts of Y-shaped polymers (solid lines) and in melts of linear polymers (dashed lines). The smooth function $\bar{G}(t)$ was obtained by the block averaging, see Eq. (4.9).

equal backbone length $\mathrm{B}_{11}$ and of (nearly) equal mass $\mathrm{B}_{13}$, respectively, we here find that the Y-shaped polymer is the slowest of the three, but the difference is smaller than in the similar comparison of T-shaped polymers in Fig. 4.2. All three $D(t)$ curves also converge 


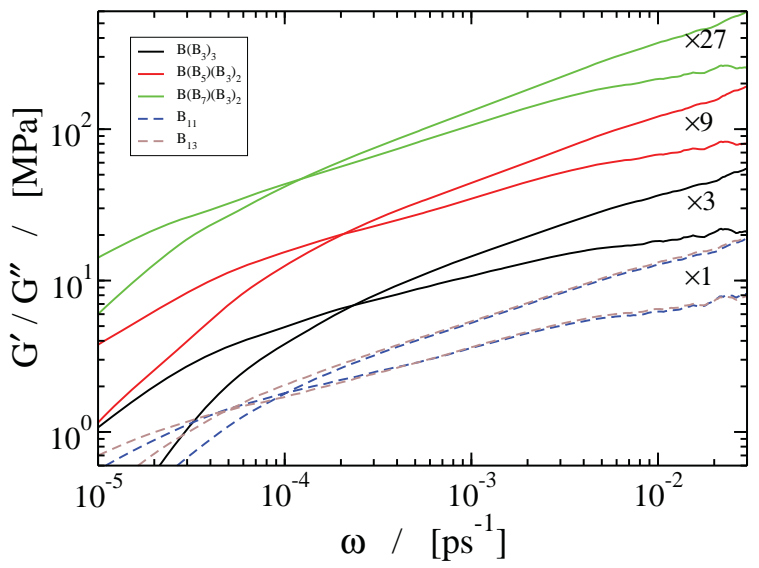

Figure 4.12: Storage and loss moduli for three Y-shaped polymers and two linear polymers. For clarity, the curves have been shifted vertically using the scaling factors indicated next to the curves.

on the same time scale, suggesting that the Y-shaped polymer behaves like a linear polymer. Because the side chains are short, the Y-shaped polymers effectively reptate in a tube like a linear polymer, with the side chain increasing the friction near one tube end, see Fig. 4.3(d).

The Y-shaped polymers also follow the short time stress relaxation of their matching linear polymers more closely, see Fig. 4.10, than the T-shaped polymers follow their matching linear polymers, in Fig. 4.6. Tube dilation by the short - and hence relatively agile - chains at the $\mathrm{Y}$ end of the polymers makes $\mathrm{B}\left(\mathrm{B}_{7}\right)\left(\mathrm{B}_{3}\right)_{2}$ relax slightly quicker than the linear polymers of equal backbone length. Like the linear polymers, but unlike the T-shaped polymers, the Y-shaped polymers deviate from power law behaviour in the short time stress relaxation. For larger times, see Fig. 4.11, the stress relaxation of the larger Y-shaped polymers slows down relative to the linear polymer of equal mass, and their respective $\bar{G}(t)$ curves cross, but this slowing down is far less pronounced than for the larger T-shaped polymers. The storage and loss moduli of the Y-shaped polymers are presented in Fig. 4.12. Unlike for the T-shaped polymers in Fig. 4.8, a clear crossing point is observed for all polymers, with the angle between the two crossing functions gradually decreasing with increasing chain length. 


\subsection{Conclusions}

In this chapter we mainly demonstrate the dynamical and rheological trend of asymmetric stars. Those asymmetric stars are built in two types, one has a short backbone and one long asymmetric arm, also called Y-shaped asymmetric star; the other one is called T-shaped asymmetric star where the backbone arms are shorter than the asymmetric arm. For both of the cases, by calculating the autocorrelation function of the asymmetric arms, as can be expected, it is found that the longer arm has a slower relaxation decay.

The asymmetric star diffusive and rheological properties are studied by measured the diffusion coefficients and stress relaxation modulus. Results are presented by the comparisons with both linear and symmetric star topologies. It is found that for asymmetric stars with the same backbone chain, by increasing the asymmetric arm length, stars feel more and more branch point constraints that they behave a slower diffusion and relaxation than corresponding linear chains, this trend is shown clearer to the asymmetric star with longer backbone chain.

In this work, it is clear to see that within our current simulation runtime, the relatively long linear or star polymers with arms in high molecular mass are not fully diffused or well relaxed. Extra simulation time are still needed for those polymers to get better statistics. 


\title{
Coarse Grain Forces in Star Polymer Melts
}

\begin{abstract}
An analysis is presented of forces acting on the centers of mass of three-armed star polymers in the molten state. The arms consist of 35 Kremer-Grest beads, which is slightly larger than needed for one entanglement mass. For a given configuration of the centers of mass, instantaneous forces fluctuate wildly around averages which are two orders of magnitude smaller than their root mean square deviations. Average forces are well described by an implicit many-body potential, while pair models fail completely. The fluctuating forces are modelled by means of dynamical variables quantifying the degree of mixing of the various polymer pairs. All functions and parameters in a coarse grain model based on these concepts are obtained from the underlying small scale simulation. The coarse model reproduces both the diffusion coefficient and the shear relaxation modulus. Ways to improve the model suggest themselves on the basis of our findings.
\end{abstract}

\subsection{Introduction}

Polymer melts and solutions exhibit dynamics on a wide range of time and length scales. The individual atoms move at time scales of picoseconds while groups of atoms move on time scales of nanoseconds. In an entangled system whole chains can move only by reptation $[36,63]$ while branched polymers move by arm retraction followed by displacements of the branch points [110]. Time scales corresponding to these processes are microseconds 
or longer. Different experimental techniques are needed to investigate different length and time scales. Neutron spin echo techniques may be used to study time scales up to a few hundred nanoseconds, while mechanical spectroscopy typically probes time scales larger than miliseconds.

Also computational studies of polymer systems require different models to investigate different time and length scales. This is not only due to computational limitations, but also results from the physicists wish to develop concepts and understanding on the basis of minimalistic models $[41,115]$. If one wants to understand chemical aspects, clearly atomistic [116] or slightly coarse grained [117] models are needed. On the other hand, if one wants to understand long time motions of polymer chains, what matters are chain connectivity and uncrossability. The minimalistic model in this case is the famous Kremer-Grest model [93], which has been used to understand how and why properties change with changing lengths of the chains. Several other models have been developed to handle even longer time scales by resorting to even coarser descriptions [44-46,118].

In recent years, we developed a model that intends to go even one step further than the models described above. Where the models described above are used to investigate material properties, we intend to devise a model that is capable of describing flow of polymer systems, or soft matter in general, in complicated geometries. This is the realm where usually computational fluid dynamics (CFD) methods are used based on one out of many constitutive models $[2,119]$ governing the relation between stresses and velocity gradients. Such models, however, are applicable only in their own limited ranges of experimental conditions. For example in polymer solutions, one may expect strong correlations between concentrations and velocity gradients $[120,121]$. Such couplings and the instabilities they give rise to are absent in the usual constitutive models, with only very few exceptions.

In order to allow for simulations of very large systems, the model that we developed is necessarily very coarse. Each polymer is represented by one single point particle [122]. Obviously these particles must be dressed with additional properties in order to take into account the response of all eliminated degrees of freedom to the ever changing circumstances provided by the configurations of the polymer positions and their histories [50]. This is reflected in the name of the model, i.e. responsive particle dynamics (RaPiD). The model is developed such that it automatically conserves the exact thermodynamic properties of the underlying 
real system. Moreover, by being a particle based simulation model, it automatically allows for concentration gradients and fluctuations necessary to initiate possible flow instabilities.

Until now we have applied the model by tuning its various parameters to experimental data [58,123-127]. In order to further test the model and investigate its conceptual soundness we want to directly calculate its various functions and parameters from data generated by a small scale simulation. The degree to which this is possible and the quality of its predictions are the subject of this paper. In Section 5.2 and Section 5.3 we describe the model and the ratio behind its particular structure. In Section 5.4 we describe how we intend to calculate the RaPiD functions and parameters from the data obtained with a small scale simulation. The small scale system in this case is a three arm star polymer melt built from KremerGrest polymers under melt conditions. In Section 5.5 we shortly describe this model and its simulation. In Section 5.6 we present our results of mapping the small scale simulation results on our coarse RaPiD model. Finally we present a summary and some conclusions in Section 5.7.

\subsection{Coarse graining}

In this section we collect some pertinent information about coarse graining. In order not to make the discussion unnecessary complicated by presenting the most general equations, we illustrate the coarse graining procedure by applying it to a melt of star polymer.

We consider a melt of $\mathrm{N}$ star polymers in a volume $\mathrm{V}$. Each polymer consists of a central bead, also called branch point, to which $f$ arms of $m$ beads each are connected. The mass of the polymer is $M=m f+1$. At the coarse level we want to describe the configuration of the melt by a set of $N$ position vectors $\vec{R}_{n}$, while all other degrees of freedom will be eliminated from the description. The elimination should be done such that as many properties as possible of the original system are described correctly by the coarse model. In particular, we would like to retain the correct dynamics and the correct thermodynamics of the system. Obviously, properties which are defined in terms of the eliminated degrees of freedom cannot be calculated from the trajectories of the coarse model.

There are two natural choices for $\vec{R}_{n}$, either the position of the branch point of star $n$, or the position of its center of mass. In this paper we adopt the second definition, i.e. $\vec{R}_{n}$ denotes 
the center of mass position vector of the $n^{\prime}$ th star. The reason for this is that with this choice $\vec{R}_{n}$ doesn't accelerate in case the total force acting on the star is zero. A second reason for this choice is that the motion of the centers of mass is much smoother than that of the branch points

Let us first consider the Hamiltonian of our system. Denoting $\vec{x}_{n i}$ the position vector of the beads, with $n$ numbering from 1 to $N$ and $i$ from 0 to $m f$ and $\vec{x}_{n 0}$ being the position vector of the central bead of the $n^{\prime}$ th star, we define

$$
\begin{aligned}
\vec{R}_{n} & =\frac{1}{M} \sum_{i=0}^{m f} \vec{x}_{n i} . \\
\vec{q}_{n i} & =\vec{x}_{n i}-\vec{R}_{n}, \quad i=1, \ldots, m f .
\end{aligned}
$$

The Hamiltonian may then readily be shown to be

$$
H=\frac{1}{2 M} \sum_{n=1}^{N} \vec{P}_{n}^{2}+\frac{1}{2} \sum_{n=1}^{N} \sum_{i=1}^{m f} \vec{p}_{n i}^{2}-\frac{1}{2 M} \sum_{n=1}^{N}\left(\sum_{i=1}^{m f} \vec{p}_{n i}\right)^{2}+V(R, q) .
$$

with

$$
\begin{aligned}
\vec{P}_{n} & ==M \dot{\vec{R}}_{n} . \\
\vec{p}_{n i} & ==\dot{\vec{q}}_{n i}+\sum_{j=1}^{m f} \dot{\vec{q}}_{n j} .
\end{aligned}
$$

$R$ denotes the set of all vectors $\vec{R}_{n}$ and $q$ the set of all vectors $\vec{q}_{n i}$; a dot over a symbol denotes a time derivative and $V(R, q)$ is the potential energy of the configuration $(R, q)$.

Since the kinetic energy does not depend on $(R, q)$, we have

$$
\dot{\vec{P}}_{n}=-\frac{\partial H}{\partial \vec{R}_{n}}=-\frac{\partial V}{\partial \vec{R}_{n}} .
$$

The general strategy of coarse graining is to manipulate the force in the right hand side such that the contributions of all $\vec{p}_{n i}$ and $\vec{q}_{n i}$ are lumped into a few functions that can easily be modeled. The first step is to separate the average force [128], so

$$
-\frac{\partial V}{\partial \vec{R}_{n}}=-\left\langle\frac{\partial V}{\partial \vec{R}_{n}}\right\rangle_{B}+\vec{G}_{n}
$$

The pointy brackets $\langle\cdots\rangle_{B}$ denote an average over all $\vec{p}_{n i}$ and $\vec{q}_{n i}$; $\vec{G}_{n}$ is what remains of the force.

In the next two sections we will describe the two contributions to the force. 


\subsubsection{Potential of mean force}

The average force introduced in Eq. (5.7) is defined as

$$
-\left\langle\frac{\partial V}{\partial \vec{R}_{n}}\right\rangle_{B}=-\frac{1}{Q} \int d q \int d p e^{-\beta H} \frac{\partial V}{\partial \vec{R}_{n}} .
$$

where $\int d q$ indicates an integral over all $\vec{q}_{n i}$ and $\int d p$ an integral over all $\vec{p}_{n i}$; moreover $Q=\int d q \int d p e^{-\beta H}$. Since the kinetic energy in Eq. (5.3) does not depend on any of $q_{n i}$, the integrals over $p$ lead to constant factors which cancel in the numerator and the denominator, so

$$
-\left\langle\frac{\partial V}{\partial \vec{R}_{n}}\right\rangle_{B}=-\frac{\partial \Phi}{\partial \vec{R}_{n}}
$$

where

$$
\Phi(R)=-k_{B} T \ln \int d q e^{-\beta V(R, q)} .
$$

We call $\Phi$ the potential of mean force. Apart from some uninteresting constants, it is equal to the free energy of the $\vec{q}_{n i}$ in a field produced by the interactions with the fixed $\vec{R}_{n}$.

Once we have completed our manipulations of $\vec{G}_{n}$ in the next section, we will find that a simulation of the coarse system samples the probability distribution

$$
P(R) \sim \exp \{-\beta \Phi(R)\}
$$

This implies that all thermodynamic properties obtained from simulations of the coarse model derive from the free energy

$$
A=-k_{B} T \ln \int d R e^{-\beta \Phi(R)} .
$$

Introducing the definition of $\Phi(R)$ we obtain

$$
A=-k_{B} T \ln \int d R \int d q e^{-\beta V(R, q)} .
$$

Apart from some constants resulting from integrals over all momenta and from a factor $\left(h^{3 N} N !\right)^{-1}$ in the partition function, $\mathrm{A}$ is the exact free energy of the microscopic system [129]. 
Whether or not we are able to obtain the exact thermodynamics from a coarse model depends on our ability to find a functional form describing $\Phi(R)$ for all configurations that contribute to the integral in Eq. (5.12), i.e. whether we are able to faithfully represent $\Phi(R)$. The usual approach is to assume that $\Phi(R)$ may be approximated as a sum of contributions from each pair of polymers

$$
\Phi(R)=\sum_{i=1}^{N-1} \sum_{j=i+1}^{N} \phi\left(R_{i j}\right) .
$$

Besides applying the pair approximation we have assumed that each contribution depends only on the distance between the two polymers that constitute the pairs. In the case of star polymers, explicit expressions for $\phi\left(R_{i j}\right)$ have been presented by Löwen, Likos and co-workers $[130,131]$. For our case, i.e. stars with a functionality of three, they write:

$$
\begin{aligned}
\phi(r) & =-\frac{5}{8} k_{B} T f^{3 / 2}\left[\ln \left(\frac{r}{\sigma_{s}}\right)-\frac{1}{2 \tau^{2} \sigma_{s}^{2}}\right], & r \leq \sigma_{s} \\
& =-\frac{5}{8} k_{B} T f^{3 / 2} \frac{1}{2 \tau^{2} \sigma_{s}^{2}} \exp \left\{-\tau^{2}\left(r^{2}-\sigma_{s}^{2}\right)\right\}, & r>\sigma_{s} .
\end{aligned}
$$

Here $\tau \sigma_{s}=1.06$ and $\sigma_{s}=\frac{4}{3} R_{g}$, with $R_{g}$ the radius of gyration of the star. In our case $f=3$.

It is clear that with a functionality as low as three, when the arms are very long, each polymer will interact with many other polymers, not just with its first neighbours. In this situation we may expect many-body interactions to be important. It is well known that it is very difficult to collect information about many-body contributions to the potential energy, free energy in our case, and to develop some intuitive understanding. Therefore, stimulated by the literature on density functional methods [132] and the work of Pagonabarraga and Frenkel [133], in recent years we have made extensive use [123, 124, 127] of an implicit many-body potential in which $\Phi(R)$ is written as

$$
\Phi_{\mathrm{FH}}(R)=\sum_{i} a\left(\eta_{i}(R)\right) .
$$

Here $\eta_{i}(R)$ is the local volume fraction of polymers at the position $\vec{R}_{i}$ defined below; $a(\eta)$ is the free energy of one polymer in a melt of volume fraction $\eta$. The contribution of the translational configurations of the centers of mass must be excluded from $\Phi_{\mathrm{FH}}(R)$. When calculating the Flory-Huggins free energy $\Phi_{\mathrm{FH}}(R)$ we treat the star polymer as being equivalent to a linear polymer consisting of $p$ Kuhn lengths, obtaining

$$
a(\eta)=k_{B} T p\left\{\frac{1-\eta}{\eta} \ln (1-\eta)-\chi \eta\right\} .
$$


The Flory-Huggins parameter $\chi$ accounts for minus the interaction energy between two Kuhn lengths.

The local volume fraction of polymers is defined as

$$
\eta_{i}(R)=\frac{1}{\rho_{\max }} \sum_{j \neq i} w\left(R_{i j}\right) .
$$

where $w\left(R_{i j}\right)$ is a normalized weight function, i.e. $\int d^{3} R w(R)=1$, implying that for a random distribution of polymers $\sum_{j} w\left(R_{i j}\right)=N / V=\bar{\rho}$. It has been argued by Trofimov et al. [134] that the sum in Eq. (5.14) should exclude the term when $j=i$.

Because of crowding near the branch point we expect that each polymer excludes the others from approaching very closely. We therefore add short-range repulsive pair potentials to the Flory-Huggins potential obtaining

$$
\Phi(R)=\Phi_{\mathrm{FH}}(R)+\sum_{i=1}^{N-1} \sum_{j=i+1}^{N} \phi^{r e p}\left(R_{i j}\right) .
$$

Notice that here it might have been better to let $R_{i j}$ be the distance between two branch points instead of the distance between two centers of mass.

\subsubsection{Transient forces}

The thermodynamic forces described in the previous section provide a reasonable estimate of the exact instantaneous forces only in case the dynamics of the eliminated degrees of freedom is much faster than that of the retained degree of freedom. As an example one may think of hard sphere colloids dissolved in a solution of small polymers which move much faster than the colloids. The forces exerted by the polymers on the colloids are well represented by depletion forces between the colloids. The remaining forces defined in Eq. (5.7) are then simple friction forces and corresponding random forces.

The situation is completely different in cases when the eliminated degrees of freedom are not much faster than the retained degrees of freedom, for example when the relaxation of the arms of a star polymer towards equilibrium is not much faster than the time scales during which the centers of mass of the polymers diffuse over length scales comparable to their sizes. In these cases, knowledge of the positions (and momenta) of the centers of mass of the stars give little or no information on the state of the eliminated degrees of freedom. The system can 
arrive at one and the same coarse state $(R, P)$ in many different ways distinguished by many different states $(q, p)$ of the arms and different instantaneous forces on the centers of mass. The only way to obtain some information about the state of the arms by interrogating the configurations of the centers of mass, is to keep track of its full history. This is why the moriZwanzig projection $[128,135]$ operator formalism leads to very complicated friction forces with memory and corresponding random forces. One way to model these friction forces is to define additional variables which give a rough description of the states of the arms and to provide a propagator which describes its dynamics. This is what we will do next.

We assume [50] that with every configuration $R$ of the centers of mass we may associate a thermodynamic equilibrium state of the arms, and that this state may be characterized by a collection of numbers $\left\{n_{0}\left(R_{i j}\right) \mid R_{i j} \leq R_{c}\right\}$. The free energy of this state is equal to the potential of mean force discussed in the previous section. We next introduce a collection of dynamical variables $\left\{n_{i j} \mid R_{i j} \leq R_{c}\right\}$ and let the deviations of these numbers from the corresponding equilibrium numbers, i.e. $\left\{n_{i j}-n_{0}\left(R_{i j}\right) \mid R_{i j} \leq R_{c}\right\}$, describe the deviation from equilibrium. We call $n_{i j}$ the contact numbers between stars $i$ and $j$, and $n_{0}\left(R_{i j}\right)$ the corresponding equilibrium contact number. Other names, like mixing number or entanglement number, have also been used. The contact numbers must be thought of as thermodynamic variables like the variables used in Landau-de Gennes types of theories. Finally, we assume that the free energy for a given configuration $R$ of the centers of mass and with the contact numbers constrained to the values $n=\left\{n_{i j}\right\}$ is given by

$$
A(R, n)=\Phi(R)+\frac{1}{2} \alpha \sum_{i=1}^{N-1} \sum_{j=i+1}^{N}\left\{n_{i j}-n_{0}\left(R_{i j}\right)\right\}^{2}
$$

where $n_{i j}$ and $n_{0}\left(R_{i j}\right)$ are non-existent when $R_{i j}>R_{c}$. The force on polymer $i$ is now

$$
\begin{aligned}
\vec{F}_{i} & =-\frac{\partial A}{\partial \vec{R}_{i}}+\vec{G}_{i}^{\prime} \\
& =-\frac{\partial \Phi}{\partial \vec{R}_{i}}+\alpha \sum_{j}\left\{n_{i j}-n_{0}\left(R_{i j}\right)\right\} \frac{d n_{0}}{d R_{i j}} \frac{\vec{R}_{i j}}{R_{i j}}+\vec{G}_{i}^{\prime}
\end{aligned}
$$

Notice that $\vec{G}_{i}^{\prime}$ is different from $\vec{G}_{i}$ in Eq. (5.7). The second term in Eq. (5.21) is called the transient force $\vec{F}_{i}^{t}$ for reasons explained in the next section. Notice that $\alpha$ may be called the strength of the transient forces. 
In the next section we will present a propagator which samples from the distribution

$$
P(R, n) \sim \exp \{-\beta A(R, n)\}
$$

Suppose we are only interested in the distribution of the centers of mass, i.e. we integrate $P(R, n)$ over all values of $n$, we obtain

$$
P(R)=\int d n P(R, n) \sim \exp \{-\beta \Phi(R)\}
$$

This means that all structural and thermodynamic properties of the system are the same as before the introduction of the transient forces.

We now turn to the propagator.

\subsection{Brownian dynamics}

We assume that with the introduction of the transient forces all memory effects are taken into account. The remaining forces $\vec{G}_{i}^{\prime}$ on polymer $i$ are then simple friction forces and corresponding Markovian random forces. Markovian means that the random forces are $\delta$-correlated in time.

Since soft matter systems are usually overdamped, we ignore momenta and make use of a simple first order propagator. For simple Markovian systems Ermak and McCammon [136] have shown how to derive the Brownian propagator from a second order Langevin equation. Here we simply pose the result

$$
d \vec{R}_{i}=-\frac{1}{\xi_{i}} \frac{\partial A}{\partial \vec{R}_{i}} d t+\frac{\partial}{\partial \vec{R}_{i}} \frac{k_{B} T}{\xi_{i}} d t+\sqrt{\frac{2 k_{B} T d t}{\xi_{i}}} \vec{\Theta}_{i} .
$$

Here $d \vec{R}_{i}$ is the displacement of particle $i$ during a time-interval from $t$ to $t+d t$. On the righthand side $\xi_{i}$ is the friction coefficient of particle $i$, which depends on the configuration at time $t$, and $\vec{\Theta}_{i}$ is a random vector associated with particle $i$ at time $t . \vec{\Theta}_{i}$ is independent of random vectors associated with other particles and independent of random vectors at other times than time $t$; moreover the components of $\vec{\Theta}_{i}$ are uncorrelated and have zero mean and unit variance. The second term in the right hand side of Eq. (5.24) is needed to ensure, together with the propagator for the contact numbers, the correct equilibrium distribution Eq. (5.22). 
In principle, we have some freedom in choosing the propagator for the contact numbers. However, if we want to have the equilibrium distribution as the stationary solution, we are forced to use [50]

$$
d n_{i j}=-\frac{1}{\tilde{\xi}} \frac{\partial A}{\partial n_{i j}} d t+\sqrt{\frac{2 k_{B} T d t}{\tilde{\xi}}} \Theta_{i j} .
$$

Here $d n_{i j}$ is the increment of $n_{i j}$ during a time interval of length $d t$. $\tilde{\xi}$ is a friction similar to the one in the propagator for $\vec{R}_{i}$. It is taken to be independent of the $n_{i j}$. In previous applications it was sometimes assumed to depend on $R_{i j}$, but in this paper it will be constant. Finally $\Theta_{i j}$ is a random number associated with the pair $i j$ at time $t$, uncorrelated from any other random numbers and having zero mean and unit variance.

In the following we will replace $\tilde{\xi}$ by $\alpha \tau$ where $\tau$ has the dimension of time (do not confuse $\tau$ with the one in Eq. (5.15)). Performing the differentiation in Eq. (5.25) we obtain

$$
d n_{i j}=-\frac{n_{i j}-n_{0}\left(R_{i j}\right)}{\tau} d t+\sqrt{\frac{2 k_{B} T d t}{\alpha \tau}} \Theta_{i j} .
$$

i.e. a simple kinetic equation with a random contribution. If we consider the contact variables as thermodynamic variables, then Eq. (5.26) resembles the evolution equation used in irreversible thermodynamics [137] with $1 / \tau$ being the Onsager coefficient.

Suppose we fix the centers of mass $\vec{R}_{i}$, then the contact numbers will gradually decay towards their equilibrium values and finally fluctuate around the latter. This is why the $\vec{F}_{i}^{t}$ are called transient forces. The physical picture is that always the contact numbers decay towards their equilibrium values, but that the latter continually change due to displacements of the particles.

Since our model consists of structureless particles, there are only two timescales in our model. One is set by the thermodynamic forces, derived from the potential of mean force, and the friction coefficient $\xi_{0}$ in Eq. (5.35). The other is similarly set by the strength of the transient forces and the corresponding friction $\tilde{\xi}$, i.e. by $\tau=\alpha \tilde{\xi}$. The rheology of the model will therefore closely resemble a two-time Maxwell model. If one wants to introduce a continuous range of characteristic times, this may be done by making $\tau$ dependent on the distance between the contacting polymers, for example according to [138]

$$
\tau\left(R_{i j}\right)=\tau e^{-R_{i j} / \lambda} .
$$


In the present application we will restrict ourselves to constant $\tau\left(R_{i j}\right)=\tau$, i.e. to $\lambda=\infty$. We leave a more realistic description for future investigations.

In case we want to calculate the parameters and functions in our coarse model, we must choose a mechanical interpretation of the contact numbers, in particular we must present a mechanical definition of $n_{i j}$. This we will do in the next section.

\subsection{Coarse parameters from small scale simulations}

In this section we describe our choices for the functions introduced in the previous section and how they will be obtained from a small scale simulation.

We start with the description of functions and parameters appearing in the Flory-Huggins free-energy. In this work we adopt the weight function introduced by Santos de Oliveira $e t$ al. [123]:

$$
\begin{aligned}
w\left(R_{i j}\right) & =C\left(R_{c}-R_{s}\right)\left(R_{c}+R_{s}-2 R_{i j}\right), & & R_{i j} \leq R_{s} \\
& =C\left(R_{i j}-R_{c}\right)^{2}, & & R_{s}<R_{i j} \leq R_{c} \\
& =0 & & R_{c}<R_{i j} .
\end{aligned}
$$

Here $C$ takes care of the normalizations. $R_{c}$ and $R_{s}$ are two adjustable parameters. In earlier work by Kindt and Briels it was found that a linear weight function performs best; the second, quadratic regime has been included to smoothen the function at $R_{i j}=R_{c}$. Since each polymer has no direct knowledge of things happening at a distance larger than a few times its radius of gyration $R_{g}$, we expect that $R_{c} \simeq 2.5 R_{g}$; around this value $R_{c}$ and $R_{S}$ may be used as adjustable parameters. Besides these, there are three more parameters, i.e. $\rho_{\max }, p$ and $\chi$, that will be treated as adjustable parameters. All three of them may be roughly estimated on the basis of their physical meaning. The range within which these parameters can be varied is therefore very small.

Next we consider the transient forces. Our intuitive picture of equilibrium between two nearby star polymers is that their arms are mixed such that there is a well defined number of contacts between the beads from one polymer with those from the other polymer. In general the number of contacts between two stars in equilibrium with each other will decrease with the distance between the stars. If we quickly bring two stars in equilibrium closer together, 
it will take some time for the stars to adjust to the new situation and to increase their mutual contacts. During this time the stars are out of equilibrium. Similarly on increasing the distance between two stars it takes some time before the stars have decreased their mutual contact number. Again, during this period the stars are out of equilibrium. From this picture it emerges that a decent definition for $n_{i j}$ might be

$$
n_{i j}=C \sum_{k} \sum_{l} X_{\Delta}\left(\left|\vec{x}_{i k}-\vec{x}_{j l}\right|\right)
$$

with

$$
\begin{aligned}
X_{\Delta}(r) & =1, \quad r \leq \Delta \\
& =0, \quad r>\Delta
\end{aligned}
$$

This indeed is the definition that we adopt. The constant $C$ will be defined below. Other definitions may be conceivable depending on the application. In particular it would be useful for linear polymers to define $n_{i j}$ such that it captures the number of topological constraints between polymers $i$ and $j$.

Given a working definition to calculate $n_{i j}$, we may calculate $n_{0}\left(R_{i j}\right)$ from an equilibrium simulation of the small scale system. To this end, for each frame of the small scale equilibrium run, we run through all pairs, calculate their $n_{i j}$ and $R_{i j}$, and put $n_{i j}$ in the bin corresponding to $R_{i j}$. Finally we average the values of $n_{i j}$ in each bin independently. In this paper we will normalize $n_{0}\left(R_{i j}\right)$ such that $n_{0}(0)=1$; this fixes the constant $C$ in Eq. (5.29). The reader must be aware that different normalizations lead to different values of $\alpha$. With this normalization, we will find that $n\left(R_{i j}\right)$ is rather insensitive for the precise value of $\Delta$ in Eq. (5.29).

In a similar way we calculate the variance $\left\langle\left(n_{i j}-n_{0}\left(R_{i j}\right)\right)^{2}\right\rangle$ for every bin, and next calculate $\alpha$ from the equipartition theorem

$$
\left\langle\left(n_{i j}-n_{0}\left(R_{i j}\right)\right)^{2}\right\rangle=\frac{k_{B} T}{\alpha\left(R_{i j}\right)} .
$$

which may be obtained with the use of Eq. (5.16). Notice that here we assume that $\alpha$ may depend on $R_{i j}$. This will actually turn out to be true. Since our intention is to only give a proof of principle of the methods presented in this paper we will ignore this fact and take $\alpha$ to be constant. 
Finally we must calculate $\xi_{i}$ and $\tau$. As already mentioned, in a simulation with fixed centers of mass the contact numbers $n_{i j}$ will initially decay towards their equilibrium values and then fluctuate around them. This allows for two possible ways to calculate $\tau$. First, we may keep track of the individual time evolutions $\Delta n_{i j}(t)=n_{i j}(t)-n_{0}\left(R_{i j}\right)$ right after the centers of mass have been fixed, and average these curves for all pairs $(i, j)$, after dividing by $\Delta n_{i j}(0)$. According to Eq. (5.26) the result will be $e^{-t / \tau}$. Because not all of the sampled functions are equally relevant from a statistical point of view, we introduce a weight $w_{i j}$ with every pair $(i, j)$ when we average over pairs, so

$$
\sum_{\langle i, j\rangle} \frac{\Delta n_{i j}(t)}{\Delta n_{i j}(0)} w_{i j}=e^{-t / \tau} .
$$

It is clear that pairs for which the absolute value of $\Delta n_{i j}(0)$ is large should have a larger weight than those for which the absolute value of $\Delta n_{i j}(0)$ is small. Therefore we adopt a weight $w_{i j}$ proportional to $\Delta n_{i j}(0)^{2}$. This then leads to

$$
\frac{\frac{1}{N_{p}} \sum_{\langle i, j\rangle} \Delta n_{i j}(t) \Delta n_{i j}(0)}{\frac{1}{N_{p}} \sum_{\langle i, j\rangle} \Delta n_{i j}(0)^{2}}=e^{-t / \tau} .
$$

Here $N_{p}$ is the number of pairs occurring in the sum over pairs. A second approach might be based on Onsager's regression hypothesis $[139,140]$, which says that equilibrium fluctuations on average decay according to the corresponding macroscopic laws. As a result

$$
\frac{\left\langle\Delta n_{i j}(t) \Delta n_{i j}(0)\right\rangle}{\left\langle\Delta n_{i j}(0)^{2}\right\rangle}=e^{-t / \tau} .
$$

Here, $\langle\cdots\rangle$ indicates an average over initial times followed by an average over pairs. Notice the similarity between both methods. Here we adopt the first method since it is computationally less demanding.

The last remaining quantity that we must specify is the friction $\xi_{i}$. Here $\xi_{i}$ is assumed to depend on the instantaneous configuration through [123]

$$
\xi_{i}=\xi_{0} \sum_{j \neq i} \sqrt{\left|n_{i j}\right| n_{0}\left(R_{i j}\right)},
$$

i.e. roughly proportional to the instantaneous contact number $n_{i j}$. The specific choice with the square root of $\left|n_{i j}\right| n_{0}\left(R_{i j}\right)$ is made to have the function equal to zero for values of $R_{i j}$ larger than $R_{c}$. The constant $\xi_{0}$ may be interpreted as the friction per contact. Although in 
principle it is possible to measure this parameter from a small scale simulation, see Padding and Briels [45], this is statistically very demanding and we will not do so. Instead we will adjust $\xi_{0}$ in order to have the diffusion coefficient of the coarse model equal to that of the small scale model.

Once all parameters have been calculated according to the methods presented in this section, the rheology of the coarse method should be equal to that of the small scale model. Of course we should not expect that the microscopic shear relaxation modulus $G(t)$ be reproduced by the coarse model in all details, but the absolute values and time scales should be roughly in agreement. For better agreement on all time scales, the coarse model should be enriched with more detail, as for example in Eq. (5.27).

\subsection{Simulations}

For the microscopic molecular dynamics (MD) simulations of the star polymer melt, we used the widely applied Kremer-Grest model with the parameter mapping for polyethylene [93]. In brief, any pair of particles separated by a distance $r$ interacts by a Weeks-Chandler-Andersen (WCA) potential,

$$
U(r)=\left\{\begin{array}{cl}
4 \varepsilon\left[\left(\frac{\sigma}{r}\right)^{12}-\left(\frac{\sigma}{r}\right)^{6}+\frac{1}{4}\right] & \text { for } \quad r<2^{1 / 6} \sigma \\
0 & \text { for } \quad r \geq 2^{1 / 6} \sigma,
\end{array}\right.
$$

with $\varepsilon / k_{B}=448 \mathrm{~K}$ and $\sigma=0.51 \mathrm{~nm}$. Bonds between adjacent beads in a chain are modelled by a finitely extensible nonlinear elastic (FENE) potential,

$$
U(r)=\left\{\begin{array}{cc}
-\frac{1}{2} k R_{0}^{2} \ln \left[1-\left(\frac{r}{R_{0}}\right)^{2}\right] & \text { for } \quad r<R_{0} \\
\infty & \text { for } r \geq R_{0},
\end{array}\right.
$$

with $k=30 \varepsilon / \sigma^{2}$ and $R_{0}=1.5 \sigma$. All particles have the same mass, $m=38.6 \mathrm{~g} / \mathrm{mol}$.

The simulations were run in Gromacs 4.6 [141], using the leap-frog stochastic dynamics integrator with a time step of $1.6 \mathrm{fs}$. This algorithm discretizes the Langevin equation of motion for particle $i$,

$$
m \ddot{\overrightarrow{x_{i}}}=-\nabla_{i} U-\gamma m \dot{\vec{x}_{i}}+\vec{f}_{i}(t),
$$

92 
with total potential energy $U$, a frictional relaxation time of $\gamma^{-1}=3.2 \mathrm{ps}$ and a Markovian random force $\vec{f}_{i}$ sampled from a Gaussian distribution. The friction and random forces collectively act as a thermostat, set by the fluctuation-dissipation theorem to maintain a temperature of $448 \mathrm{~K}$.

The simulation box contained 300 star polymers, each consisting of three arms of 35 beads - corresponding to approximately one entanglement length - connected to a central bead. The 31800 beads were confined in a cubic simulation box with periodic boundary conditions, at a bead number density of $0.85 \sigma^{-3}$. Initial boxes were created by inserting randomly generated chains at random positions in the box, taking care to avoid too large overlaps. The box was then relaxed by energy minimization, followed by an equilibration run of typically $10^{8} \mathrm{MD}$ steps. Production runs to collect data for analysis lasted about $10^{8}$ MD steps each. In several simulations the polymer centers of mass (COM) were frozen by using rigid body translations to move their COMs back to their original positions after every integration step.

RaPiD simulations were also done in cubic boxes with edges of length equal to $17.06 \mathrm{~nm}$, and containing 300 particles.

\subsection{Results}

In order to appreciate the character of the forces on centers of mass in a star polymer melt, we present in Fig. 5.1 a time sequence of the force in $x$-direction on the center of mass of a particular star polymer in a melt in which the centers of mass of all stars were fixed. The initial configuration was taken from an equilibrium simulation with all degrees of freedom, including those of the centers of mass, free to move. It is seen that the instantaneous force fluctuates between $-2000 \mathrm{pN}$ and $+2000 \mathrm{pN}$. The average is equal to $21.1 \mathrm{pN}$. Obviously it will be very difficult to extract the average forces from our simulations. This can be appreciated even better from a glance of Fig. 5.2. In this figure we have plotted the average forces in $x, y$, and $z$-directions for all 300 stars in the box with frozen center of mass, calculated from the first $7.5 \times 10^{7}$ time steps after equilibration, along the horizontal axis, and those from the next $7.5 \times 10^{7}$ time steps along the vertical axis. Equilibration lasted $3 \times 10^{7}$ time steps. Averaging was done by picking frames every hundredth time step and calculating the mean. In 


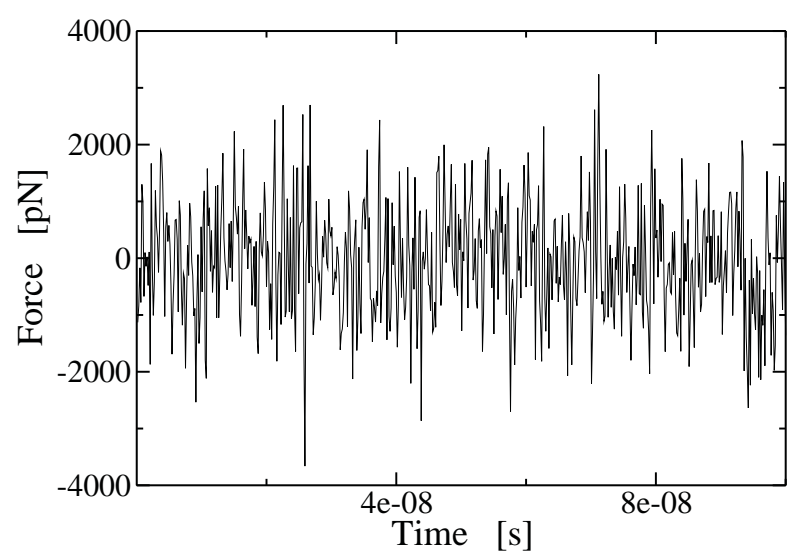

Figure 5.1: Time sequence of forces in $x$-direction on one particular star polymer in a melt with all centers of mass fixed.

the ideal case Fig. 5.2 would have been a straight line along the diagonal with positive slope. It is clear that the data still scatter substantially. Notice that for the final averages, based on $1.5 \times 10^{8}$ time steps, the scattering is less by a factor of $\sqrt{2}$.

Before discussing the average forces, let us take a look at Fig. 5.3, in which $n_{0}(r)$ is plotted against the distance between two stars. The inset shows the results for five different values of $\Delta$ in Eq. (5.29). Obviously, $n_{0}(r)$ increases with increasing values of $\Delta$. In the main part of the plot, $n_{0}(r) / n_{0}(0)$ is plotted for all five values of $\Delta$. Clearly all results are nearly equal, indicating that our normalized definition of the contact numbers $n_{i j}$ is rather insensitive to the precise value of $\Delta$. Also shown in this figure is the analytic representation that we fitted to the experimental data:

$$
n_{0}(r)=\exp \left\{-\left(\frac{r / R_{g}}{1.357}\right)^{2}\right\} .
$$

The Gaussian form was taken from Akkermans and Briels [49]. From the plot it seems safe to use a cutoff value $R_{c}=3 R_{g}$. This is somewhat larger than the $2.5 R_{g}$ used in previous applications of the RaPiD model.

We now consider the description of the average forces by means of the extended FloryHuggins model Eq. (5.19). In a first step we ignore the repulsive contributions, which leaves us with the pure Flory-Huggins free energy $\Phi_{\mathrm{FH}}$ and its adjustable parameters, $p, \chi, \rho_{\max }, R_{s}$ 


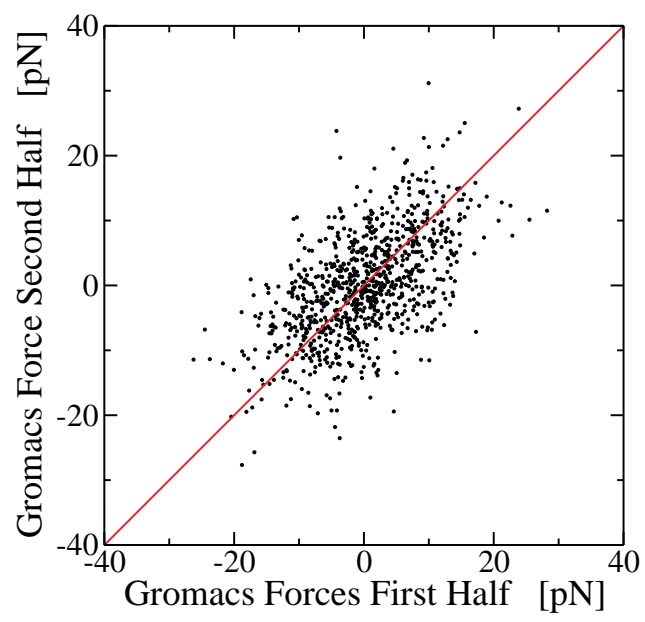

Figure 5.2: All 900 Gromacs forces on the centers of mass of 300 star polymers in a melt with fixed centers of mass. Values along the vertical axis are averaged over $7.5 \times 10^{7}$, time steps of length $\Delta t=1.2 \times 10^{8} \mathrm{fs}$, while those along the horizontal axis are averaged over the preceding $7.5 \times 10^{7}$ time steps.

and $R_{c}$. On the basis of their physical meanings, $n_{0}(r)$ and $\omega(r)$ must have very similar cutoff radii. In order to restrict the number of adjustable parameters we therefore choose $R_{c}=3 R_{g}$ also for the weight function. Finally we choose $R_{s}=1 R_{g}$ in order to have a smooth weight function as shown in Fig. 5.4

We are now left with three adjustable parameters. Since there are no attractive forces in our Gromacs simulation and the repulsions are very steep, almost all of $\Phi_{\mathrm{FH}}$ must come from entropy, which means that $\chi$ must be zero or slightly negative. Moreover, writing $\rho_{\max }=r \bar{\rho}$, with $\bar{\rho}$ the overall number density, $r$ must take values between 1.5 and 2 . This follows from the fact that the density in the Kremer-Grest system is roughly half the melt density. Finally, also $p$ can be roughly estimated according to $p=3 p_{\text {arm }}-2$, with

$$
p_{\text {arm }}=\frac{\left\langle R_{\text {arm }}^{2}\right\rangle}{b^{2}}=\frac{\left\langle R_{\text {arm }}^{2}\right\rangle}{C_{\infty}^{2} l^{2}},
$$

where $R_{\text {arm }}$ is the branch-end distance of one arm. From our simulations we found $\sqrt{\left\langle R_{\text {arm }}^{2}\right\rangle}=$ $6.8 \sigma$. Using the values $l=0.97 \sigma$ and $C_{\infty}=1.80$ from the paper of Kremer and Grest [93] 


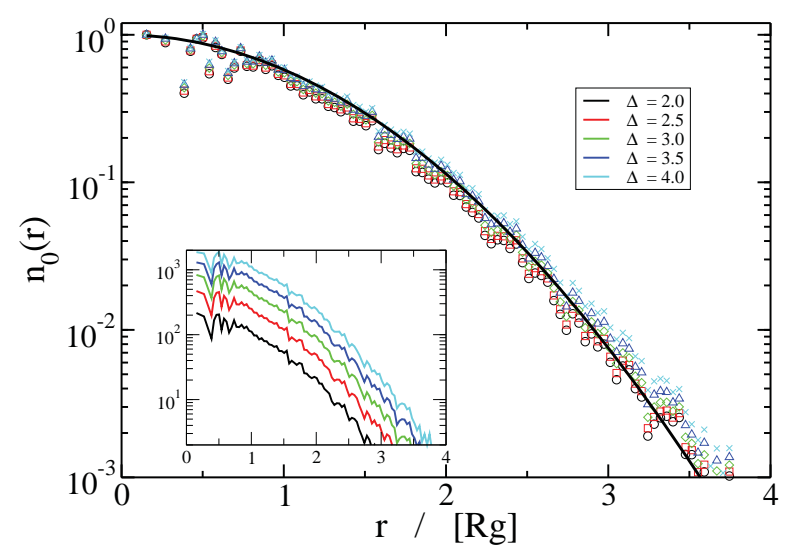

Figure 5.3: Average numbers of contacts $n_{0}(r)$ as a function of the distance between two stars for several values of $\Delta$ in Eq. (5.29). The black line is a fit to the data.

we get $p \simeq 43$. A somewhat smaller value of $p \simeq 40$ is obtained when the star is treated as a linear chain with $R_{g}=4.33 \sigma$.

In order to determine $\rho_{\max }, \chi$ and $p$ we have performed a sequence of optimizations. We fixed $\rho_{\max }$ to some particular value and for a sequence of values for $\chi$ we minimized

$$
D^{2}=\sum_{i=1}^{2700}\left(F_{\mathrm{FH}}^{i}-F_{G}^{i}\right)^{2}
$$

by adjusting $p$. Here $F_{\mathrm{FH}}^{i}$ is the $i^{t h}$ force obtained with the Flory-Huggins model and $F_{G}^{i}$ the $i^{t h}$ force obtained with the Gromacs data. The resulting values of $p$ as a function of $\chi$ are shown in Fig. 5.5 for several values of $\rho_{\max }$. The corresponding qualities of fit $F Q=D_{\min } / \sqrt{\left\langle F_{G}^{2}\right\rangle}$, with $D_{\min }^{2}$ being the minimum of $D^{2}$ and $\left\langle F_{G}^{2}\right\rangle$ the average of all 2700 forces squared obtained from the Gromacs simulations with frozen centers of mass (three boxes with 300 particles each), are shown in Fig. 5.5. Clearly, with all values of $\rho_{\max }$, the quality of fit becomes bad for positive Flory-Huggins parameters $\chi$. Moreover, if one wants the number of Kuhn lengths $p$ to be around 40, unrealistically large values of $\rho_{\max }$ are needed with decreasing values of $\chi$. The only physically reasonable choice of parameters, still with a favourable value of $F Q$, seems to be $\rho_{\max }=1.9 \bar{\rho}, \chi=0$ and $p=43$, in close agreement with the initial estimates.

With $\Phi_{\mathrm{FH}}(R)$ now defined, we turn our attention to $\phi^{\text {rep }}\left(R_{i j}\right)$ in Eq. (5.19). Assuming a 


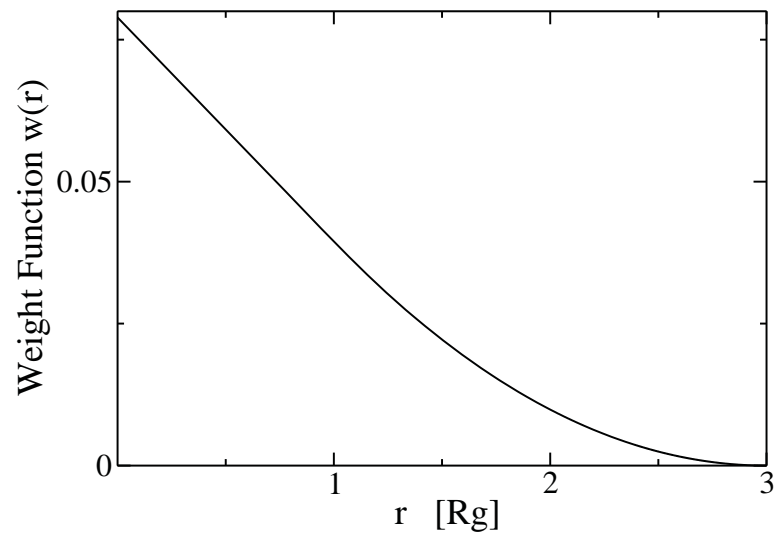

Figure 5.4: Weight function $w(r)$ used to calculate local volume fraction (Eq. (5.18)).

Gaussian function with two parameters

$$
\phi^{r e p}\left(R_{i j}\right)=a \exp \left\{-\left(R_{i j} / b\right)^{2}\right\}
$$

and replacing the Flory-Huggins forces in Eq. (5.41) by those obtained with the extended Flory-Huggins model, we again minimized $D^{2}$, with fixed Flory-Huggins parameters, and found $a=1.194 k_{B} T$ and $b=1.283 R_{g}$ with a quality if fit $F Q=0.78$. To appreciate this result, we have plotted in Fig. 5.6 left panel the model forces $F_{\text {model }}^{i}$ against the Gromacs forces $F_{\mathrm{G}}^{i}$. For comparison we show in Fig. 5.6 right panel the same plot, with the model forces calculated using the pair potential of Löwen, Likos and co-workers. Clearly the extended Flory-Huggins model represents the actual average forces much better than the pair model. In Fig. 5.7 we show the radial distribution functions obtained with the various models. As is clear from the figure, the extended Flory-Huggins model describes the Gromacs data very well, while the pair potential model is only marginally successful.

We now turn to the transient forces. As we mentioned in the previous section, we will use the equipartition theorem Eq. (5.31) to calculate $\alpha\left(R_{i j}\right)$ from the Gromacs data. In deriving Eq. (5.31) from the probability distribution Eq. (5.22), it was assumed that $n_{i j}$ can take negative values, as they can do in the coarse model. With the definition of $n_{i j}$ given in Eq. (5.29), however, the Gromacs $n_{i j}$ can only take positive values. For small values of $R_{i j}$ this is actually not a restriction, because indeed the $n_{i j}$ are Gaussian distributed around their 

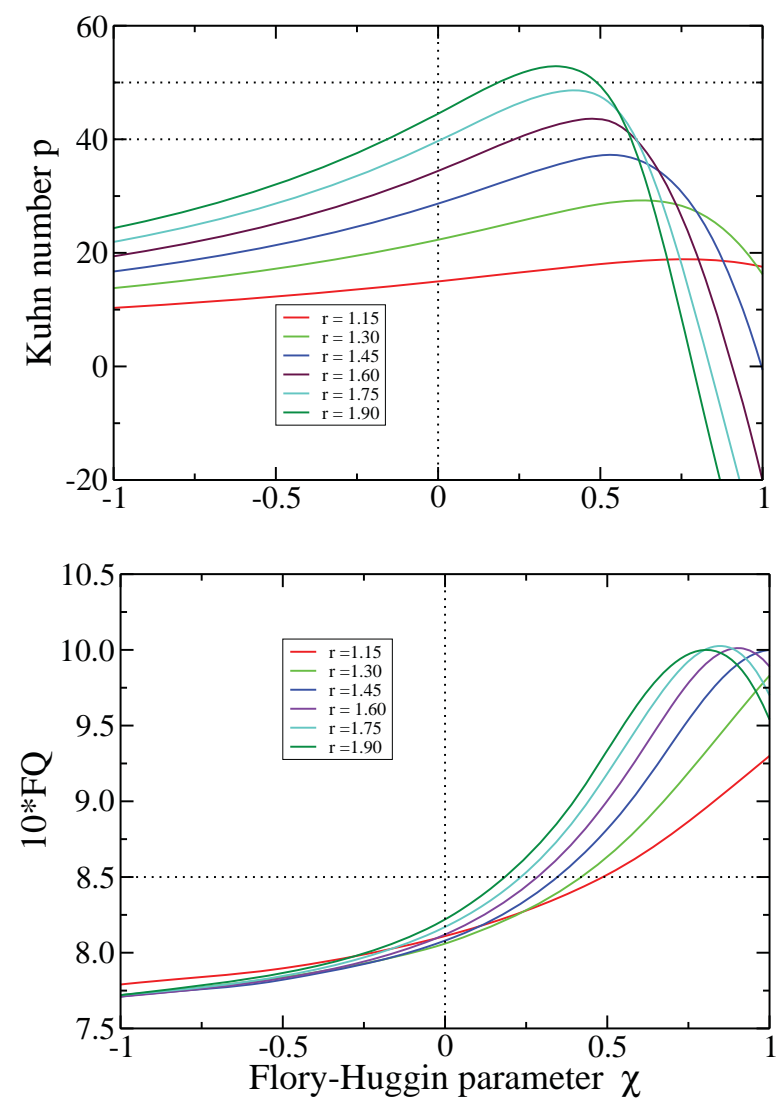

Figure 5.5: Left panel: optimal number of Kuhn lengths as a function of $\chi$ for various values of $r=\rho_{\max } / \bar{\rho}$. Right panel: qualities of fit $F Q$ (multiplied by 10) corresponding to the curves in the left panel.

mean values $n_{0}\left(R_{i j}\right)$. With increasing values of $R_{i j}$, the distribution of the Gromacs $n_{i j}$ begins to resemble a Poisson distribution, with the result that $\alpha\left(R_{i j}\right)$ cannot be calculated from the equipartition theorem anymore. In Fig. 5.8 left panel we have plotted $n_{0}$ together with $n_{0}+\sqrt{k_{B} T / \alpha}$ and $n_{0}-\sqrt{k_{B} T / \alpha}$ as a function of $r$. At a distance somewhat larger than $R_{g}$, $n_{0}-\sqrt{k_{B} T / \alpha}$ drops below zero. This is a clear indication that the Gaussian assumption does not apply anymore to the distribution of Gromacs contact numbers. At the same time, the statistics is rather bad for small distances since only very few pairs exist at these distances. 

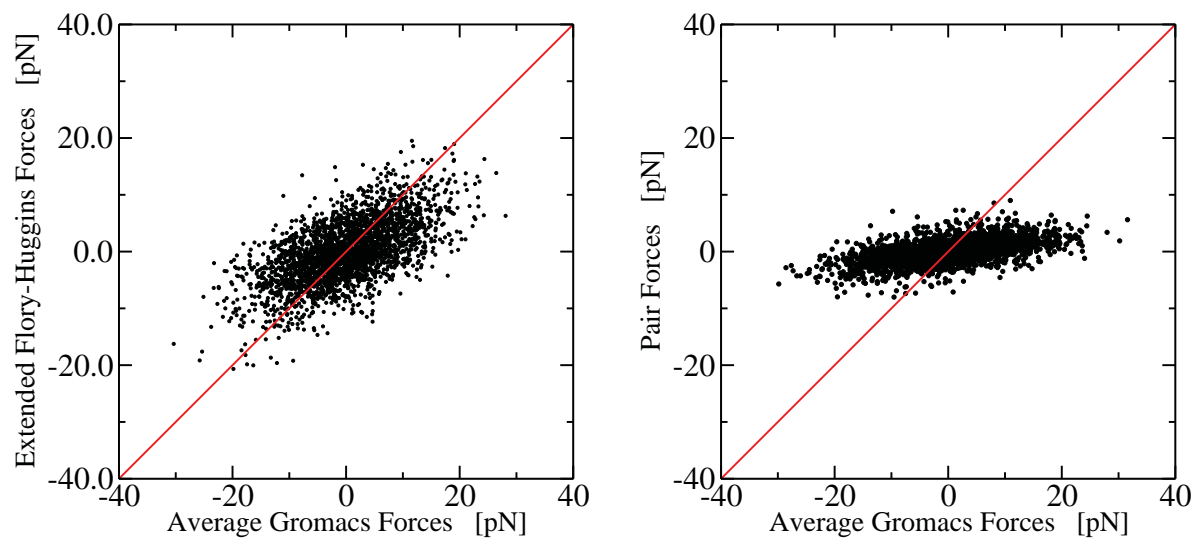

Figure 5.6: Left panel: all 2700 forces on the center of mass of 900 star polymers in 3 boxes of 300 star polymers each, calculated with the extended Flory-Huggins model, along the vertical axis, and averaged over a Gromacs run with fixed centers of mass of $1.5 \times 10^{8}$ time steps along the horizontal axis. Right panel: same as in left panel with extended FloryHuggins forces replaced by pair forces from reference [131].

We therefore decided to take for $\alpha$ its value at $r=R_{g}$. In Fig. 5.8 right panel we have plotted the radial contact number density $4 \pi r^{2} \rho g(r) n_{0}(r)$ as a function of the distance to a star in the origin. Clearly most contacts exist with the stars at distances between one and two $R_{g}$. This corroborates our choice to use $\alpha=\alpha\left(R_{g}\right)$. The values of $\alpha(r)$ are shown in Fig. 5.9, together with a fit $\alpha(r) \simeq 1.94+2.168\left(r / R_{g}\right)^{2.12}$, from which we obtain $\alpha\left(R_{g}\right)=4.0$.

The final two parameters to be calculated are $\xi_{0}$ and $\tau$. As already mentioned in the previous section, we will use Eq. (5.33) to calculate $\tau$. The left hand of this equation will be called $C(t)$. Before extracting $\tau$, we must decide about the time scale on which we should calculate $C(t)$. According to our general picture of the dynamics of the stars, the arms relax towards equilibrium dictated by the collection $\left\{n_{0}\left(R_{i j}\right)\right\}$. These numbers will have changed, however, as soon as all stars have diffused appreciably, let's say over distances of about $0.1 \sim 0.2 R_{g}$. From our Gromacs data we found that such displacements correspond to about 0.5 ns. In Fig. 5.10 we have plotted $C(t)$ for times up to $1 \mathrm{~ns}$. We observe two exponential decays, the first of which corresponds to our $\tau$. From a fit with two exponents we obtain 


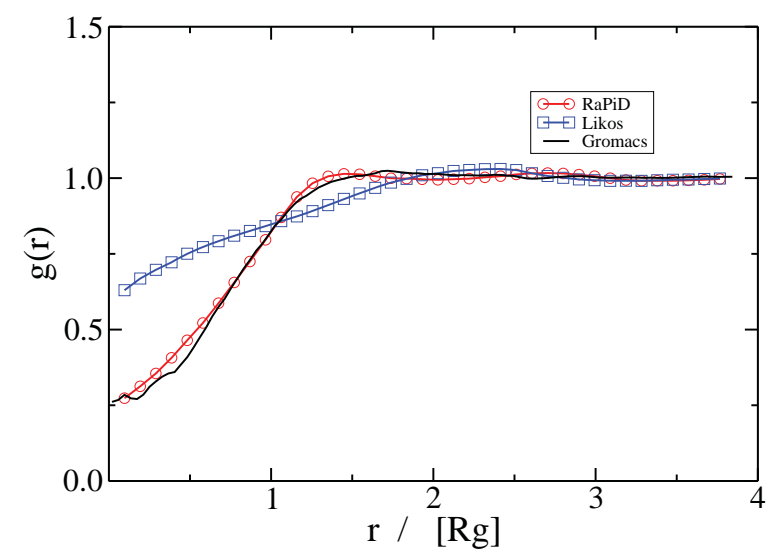

Figure 5.7: Radial distribution function for centers of mass, obtained from Gromacs simulation (black line), from RaPiD simulation (red line circles) and from a simulation (blue line square) with the pair potential from reference [131].

$C(t)=0.7762 \times e^{\left(-t / 0.12 \times 10^{-9}\right)}+0.14 \times e^{\left(-t / 3.5 \times 10^{-9}\right)}$ and so $\tau=0.12 \mathrm{~ns}$.

The friction per contact, $\xi_{0}$, is extremely difficult to measure from small scale simulations. We therefore choose to follow a pragmatic route by adjusting $\xi_{0}$ until the progressive diffusion coefficient

$$
D(t)=\frac{1}{6 t}\left\langle\left(\vec{R}_{i}(t)-\vec{R}_{i}(0)\right)^{2}\right\rangle
$$

is well represented by the coarse model. In Fig. 5.11, $D(t)$ is plotted as a function of time, once using RaPiD data and once using Gromacs data. With $\xi_{0}=1.06 \times 10^{-11} \mathrm{Kg} / \mathrm{s}$ the final diffusion coefficient $D=\lim _{t \rightarrow \infty} D(t)$ is well represented by the RaPiD model. Of coarse at the very early times RaPiD cannot reproduce the correct dynamics because it is driven by a sequence of processes at time scales that have been replaced by a Markovian friction plus corresponding noise. Also at the intermediate times RaPiD is not very successful.

At this point, all parameters of the RaPiD model have been calculated from an underlying small scale Gromacs simulation. If the model captures the real physics it should now reproduce all physical properties without any additional parameter tuning. Therefore we calculated 

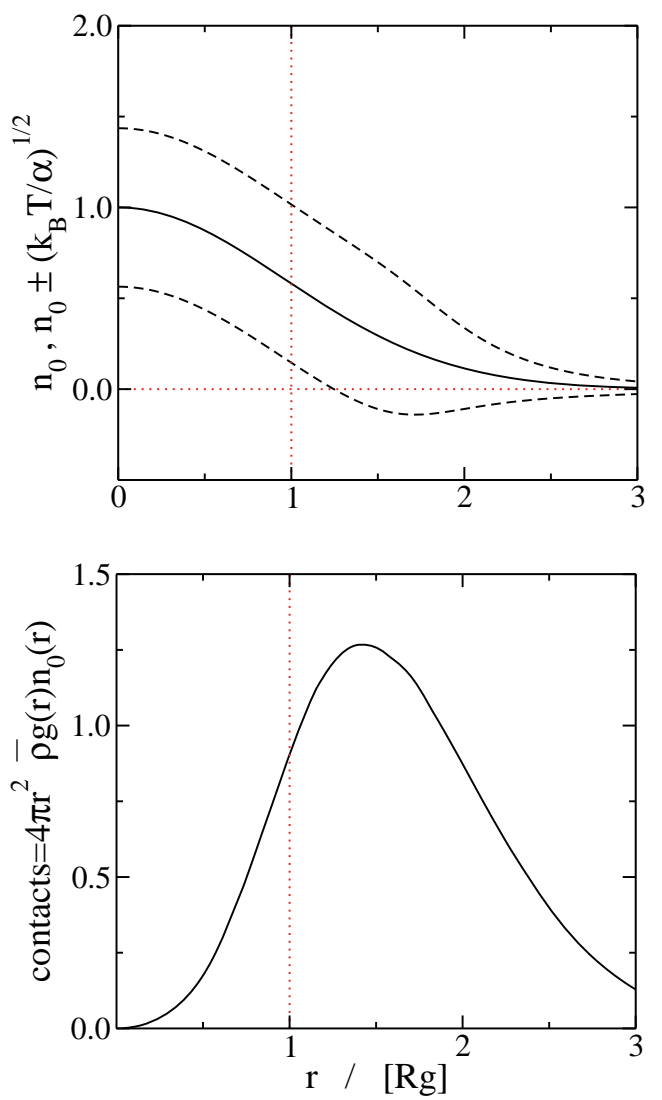

Figure 5.8: Left panel: average contact number $n_{0}(r)$ as a function of $r$ (black line) and $n_{0}(r) \pm \sigma_{n}$ with $\sigma_{n}=\sqrt{k_{B} T / \alpha}$ (dotted lines). Right panel: total number of contacts $4 \pi r^{2} \bar{\rho} g(r) n_{0}(r)$ as a function of $r$.

the shear relaxation modulus

$$
G(t)=\frac{V}{k_{B} T}\left\langle\sigma_{x y}(t) \sigma_{x y}(0)\right\rangle,
$$

with $\sigma_{x y}$ being the $x y$ element of the stress tensor calculated according to

$$
\sigma_{x y}=-\frac{1}{V} \sum_{i j}\left(R_{i, x}-R_{j, x}\right) F_{i j, y} .
$$

In the last equation, $R_{i, x}$ is the $x$-coordinate of the position vector of particle $i$ and $F_{i j, y}$ is 


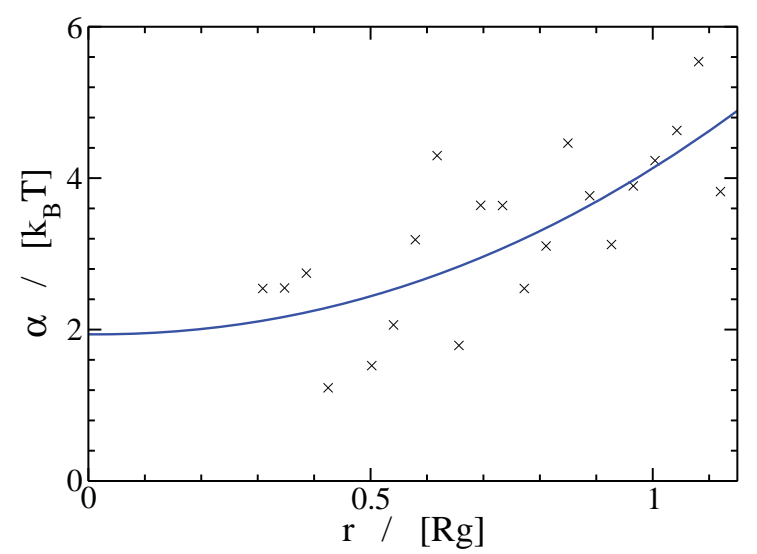

Figure 5.9: Strength of transient forces $\alpha$ as a function of $r$.

the $y$-component of the force exerted by particle $j$ on particle $i$. The results are shown in Fig. 5.12. Clearly, the results obtained with the Gromacs data are very noisy. This is a well known fact with all small scale simulations, which is due to the fact that the stress-stress auto-correlation function in Eq. (5.46) is calculated from just one single, heavily fluctuating quantity, as opposed to auto-correlation functions of single particle properties, which fluctuate less wildly and moreover may be averaged over all particles in the box. The shear relaxation modulus obtained with the coarse model ignoring the transient forces, by always putting $n_{i j}=$ $n_{0}\left(R_{i j}\right)$, reproduces the Gromacs data at long times surprisingly well. At intermediate times, clearly the transient forces are needed to reproduce the Gromacs $G(t)$. The full RaPiD curve roughly exhibits two relaxations. This should not be surprising since with $\sigma_{x y}=\sigma_{x y}^{\text {th }}+\Delta \sigma_{x y}$ and $\left\langle\Delta \sigma_{x y}(t) \sigma_{x y}^{t h}(0)\right\rangle=\left\langle\Delta \sigma_{x y}(0) \sigma_{x y}^{t h}(t)\right\rangle=0$ we obtain

$$
G(t)=\frac{V}{k_{B} T}\left\{\left\langle\sigma_{x y}^{t h}(t) \sigma_{x y}^{t h}(0)\right\rangle+\left\langle\Delta \sigma_{x y}(t) \Delta \sigma_{x y}(0)\right\rangle\right\}
$$

Here $\sigma_{x y}^{t h}$ is the $x y$-stress contribution from the thermodynamic forces and $\Delta \sigma_{x y}$ the remaining part, contributed by the transient forces. The vanishing of the cross correlations $\left\langle\Delta \sigma_{x y}(t) \sigma_{x y}^{t h}(0)\right\rangle$ is due to the fact that with every configuration of the center of mass the transient forces fluctuate around zero. Even when the order of magnitude and the covered time regimes are correct it is clear that the model is too simple to capture all the details of the shear relaxation modu- 


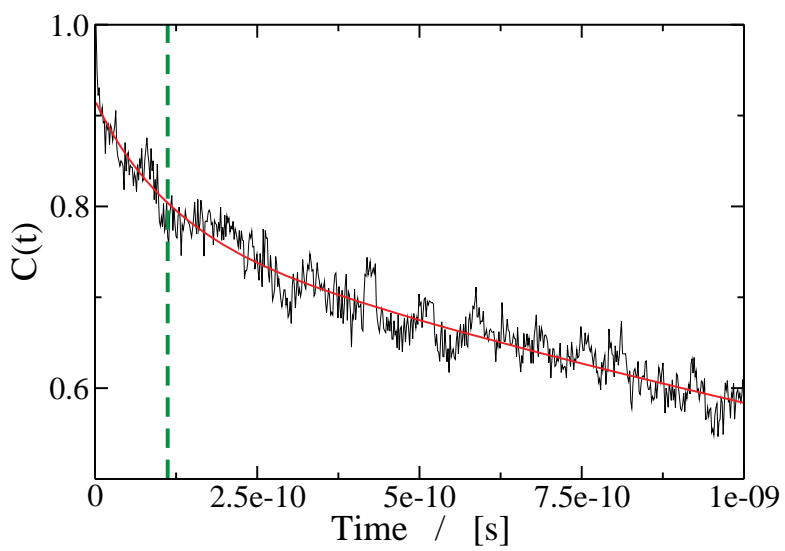

Figure 5.10: Decay curves $C(t)$ (left hand side of Eq. (5.33)) as a function of time lapse since freezing the centers of mass motion. The dashed line indicates the time when in the free box the centers of mass have moved over $0.15 R_{g}$.

lus at intermediate times. In previous applications of the RaPiD model, we have shown that with some simple adjustments, like in Eq. (5.27) or allowing for deformation of the coarse particle [127], very good results may be obtained. Here we have shown that the successes of the RaPiD model are not just sheer luck, but that all parameters indeed have a well defined physical meaning. Our limitations in this paper have basically been due to difficulties to run sufficiently long small scale simulations.

\subsection{Summary and conclusions}

We have presented a method to calculate all functions and parameters in the responsive particle model (RaPiD) from an underlying small scale simulation. RaPiD is a coarse grained model in which every polymer is represented by a single point particle dressed with additional variables to describe the dominant effects of the eliminated degrees of freedom. In order to conserve both the thermodynamic and the dynamic properties of the original model, forces are considered to consist of two contributions. The first contribution is taken to be the average forces for given, instantaneous positions of the particles. The remaining contribution 


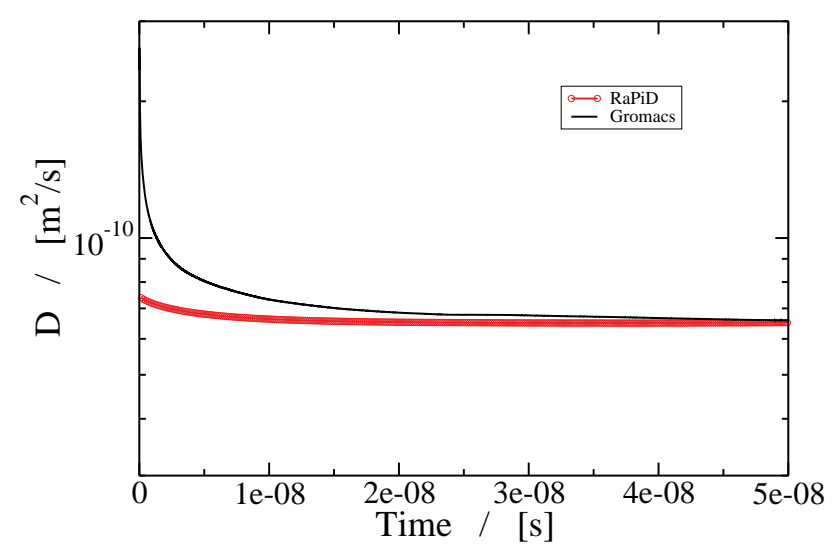

Figure 5.11: Progressive diffusion coefficient as a function of time from Gromacs run (black line) and from RaPiD run (symbols).

is modelled by an additional set of dynamical variables for each pair within a certain cutoff distance.

The average forces are taken to derive from a potential with implicit many-body contributions based on the Flory-Huggins model, to properly take care of the entropy of the eliminated degrees of freedom. With a previously suggested definition of local volume fractions all parameters in the model may be estimated on the basis of their physical meaning. With only very small tuning of these parameters around the estimated values very good agreement between actual and model forces was obtained. Similar agreement could not be obtained with the best effective pair potential model available. In order to treat the pair model on the same footing as ours, we have optimised all parameters in the pair model to achieve good agreement with the actual forces, but this led to unphysical results. We conclude that many-body terms are an important ingredient in coarse grain models for polymer melts and solutions.

The fluctuating forces are larger than the average forces by two orders of magnitude. This fact alone is enough to conclude that it will be impossible to describe both the thermodynamic properties and the dynamic properties correctly on the basis of a simple conservative forcefield involving only the positions of the polymers. In the RaPiD model the fluctuating forces are described by so called contact numbers, whose dynamics brings into the simulation the 


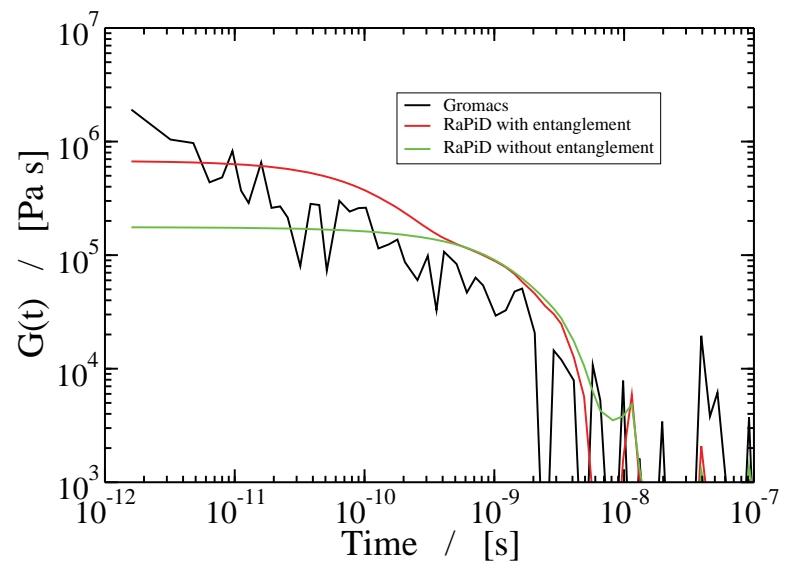

Figure 5.12: Shear relaxation modulus from Gromacs run (black line) and from RaPiD run (red line); the green line represents the contribution from the conservative forces.

memory effects resulting from the elimination of the internal degrees of freedom of the polymers. We have measured the root mean square deviations from their equilibrium values and the corresponding decay times of these contact numbers. This left us with one parameter that we adjusted to have the diffusion coefficient correct. The shear relaxation modulus as a function of time was next found to be correctly reproduced by the coarse model, albeit not in all its details.

In summary we conclude that splitting forces into average forces and transient forces is a very promising concept. Average forces require many-body contributions, which can be modelled very well. The present implementation of the transient forces performs rather well. Our calculations have revealed, however, that there is still quite some room for amelioration of the modelling of the transient forces. We mention three possible ways to attack this problem. First, it is conceivable that, depending on the application, better definitions exist for the variables $n_{i j}$. Second, other distributions than the Gaussian distribution for the $n_{i j}$ should be considered. Finally, ways to implement a range of characteristic times into the dynamics of the $n_{i j}$ should be developed; one such way was already implemented by Sprakel et al. [138], others may be conceivable. 



\section{Summary}

In this thesis the properties of star and linear polymer melts have been studied by coarse grained simulations. Depending on the architecture, polymers are observed to show different dynamical and rheological properties. The aim here is to reveal how architecture affects polymer behaviour. The results obtained from this work will not only contribute to a fundamental understanding of the character of star polymeric systems, but also possibly help to design industrial architecturally complex polymer materials with specific macroscopic properties. Simulations are carried out with coarse-grained method at multiple levels: in Chapters 2 through 4 the mesoscale Twentanglement model introduced by Padding and Briels, where each polymer is represented by a chain of consecutive soft blobs; in Chapter 5 both Molecular Dynamics (MD) simulations at atomistic level and highly coarse grained Responsive Particle Dynamics (RaPiD) simulations, in which a polymer is reduced to a single particle.

In Chapter 2, the Twentanglement algorithm for mesoscopic simulations of polymers is extended to study three-armed polymers. This technique simulates polymers as short chains of soft blobs, representing polymer segments, with bond crossings prevented by uncrossibility constraints. After defining the linking structure between branch points and linear arms, a test was conducted on two linear chains connected by a branch point to ensure that the entanglements can slip over the branch point from one arm to the other. Additional geometric checks were implemented for entanglement points slipping over a branch point connecting three arms. In this chapter, a star with three moderately entangled linear arms is studied as an example to test the method. The segmental diffusions are calculated, where it is found that the branch point diffuses the slowest and the free ends move the fastest. The same trend is observed from the calculation of Rouse mode coordinates. The diffusive behaviour of threearmed stars is found to be close to that of a linear chain with the same molecular weight, while in terms of the rheology the star polymer behaves like its longest linear backbone chain.

Stars with various arm lengths are studied by Twentanglement model in Chapter 3. Scaling laws for linear chain diffusion and zero shear viscosity as functions of molecular mass are found in quantitative agreement with the experimental observations and theoretical predictions. These results underline that the length of the linear polymers and stars studied in 
this model are in the crossover from the Rouse model to reptation theory. Analysis of the dynamical and rheological properties of melts of star polymers are made and compared with those of linear polymers. By increasing the arm length, the star polymers gradually show a slower diffusion than both matching linear chains, as well as a delayed relaxation. The differences between linear and star viscoelastic behaviour can also be seen from their storage and loss moduli; linear chains show a wider wedge than the corresponding star polymer after the crossing frequency of those two curves. Furthermore, in this chapter the zero shear viscosities of star melts are calculated from their stress moduli. The observed exponential scaling law in the arm molecular mass of star polymer shows a reasonable agreement with experimental and theoretical investigations for very large star polymers presented in the literature.

In Chapter 4, the dynamics and viscoelastic behaviour of melts of asymmetric threearmed polymers has been simulated by the TWENTANGLEMENT method. A short side arm, well below the entanglement length, shows already a notable change in the properties of the melt. The presence of this agile side chain allows for relatively fast local stress relaxations and dilates the tube constraining the backbone. For long side chains, approaching the entanglement length, the relaxation dynamics of the melt shows the hall-marks of the arm retraction process. The rarity of this process, occurring about once per $0.4 \mu$ s per arm, sets the slowest time scale of the melt.

The investigation of polymer systems at different time and length scales requires different coarse graining levels. Chapter 5 presents a highly coarse grained model, Responsive Particle Dynamics (RaPiD), where each polymer is represented by one particle. In this model, forces act on the centers of mass of each polymer. Those coarse grained forces can be split into conservative interactions described by the extended Flory-Huggins potential, 'transient' forces depending on the contacts between polymers to represent entanglement effects, and random thermal forces. In this work, instead of the top-down approach currently employed to tune the RaPiD model, the parameterization is obtained in a bottom-up approach as well as considerations of polymer concepts in physics. The Kremer-Grest model is applied for the atomistic or microscopic simulation of three-armd star polymers with approximately one entanglement mass per arm. The radial distribution function generated by RaPiD reproduces the small scale simulation data very well, which indicates that the extended Flory-Huggins potential describes the statistical properties of this star system very well. The diffusion co- 
efficient in $\mathrm{RaPiD}$ is tuned by adjusting the friction per contact. The shear stress modulus as calculated in RaPiD, without adjusting additional parameters, is in reasonable agreement with the small scale simulations. This work proves that a many-body model is capable of describing the system dynamics very well. Suggestions on how to improve the agreement are made at the end of this chapter. 



\section{Samenvatting}

In dit proefschrift zijn de eigenschappen van smelten van lineaire en ster-vormige polymeren bestudeerd met grof-korrelige simulaties. Polymeren vertonen verschillend dynamisch en reologisch gedrag afhankelijk van hun architectuur. Het doel hier is zichtbaar maken hoe architectuur het polymeergedrag beïnvloedt. De resultaten verkregen in dit werk zullen niet alleen bijdragen aan een fundamenteel begrip van het karakter van ster-polymeer systemen, maar mogelijk ook helpen bij het ontwerpen van industriële architectonisch complexe polymeermaterialen met specifieke macroscopische eigenschappen. Simulaties zijn uitgevoerd met grof-korrelige methodes op meerdere niveaus: in Hoofdstuk 2 tot en met 4 het TWENTANGLEMENT model geïntroduceerd door Padding en Briels, waar elk polymeer wordt gerepresenteerd door een keten van zachte 'blobs'; in Hoofdstuk 5 zowel Moleculaire Dynamica (MD) simulaties op atomair niveau en extreem grof-korrelige Responsive Particle Dynamics ( RaPiD) simulaties, waarin een polymeer gereduceerd is tot een enkel deeltje.

In Hoofdstuk 2 wordt het TWENTANGLEMENT algoritme voor mesoscopische simulaties van polymeren uitgebreid voor de bestudering van drie-armige polymeren. Deze techniek simuleert polymeren als korte ketens van zachte "blobs", die polymeersegmenten representeren, met bindingsdoorsnijdingen voorkomen door niet-doorsnijdings restricties. Nadat de verbindingsstructuur tussen een vertakkingspunt en lineaire armen is gedefinieerd, werd een test uitgevoerd op twee lineaire ketens verbonden door een vertakkingspunt om er zeker van te zijn dat de 'entanglements' (d.w.z. verknopingspunten waar twee polymeren aan elkaar haken) over een vertakkingspunt kunnen glijden van de ene arm naar een andere. Aanvullende geometrische testen werden geïmplementeerd voor verknopingspunten die glijden over een vertakkingspunt verbonden met drie armen. In dit Hoofdstuk wordt een ster met drie matig verknoopte armen bestudeerd als een voorbeeld om de methode te testen. De diffusies van segmenten zijn berekend, waarbij gevonden wordt dat het vertakkingspunt het traagst diffundeert en de vrije einden het snelst. De zelde trend wordt gezien in de berekening van Rouse mode coordinaten. Het diffusieve gedrag van drie-armige sterren blijkt dicht te liggen bij dat van een lineaire keten met hetzelfde moleculaire gewicht, terwijl in termen van de reologie het ster-polymeer zich gedraagt als zijn langste lineaire ruggengraat. 
Sterren met verscheidene armlengtes nabij de reptatie-overgang worden bestuurd met het TWENTANGLEMENT model in Hoofdstuk 3. Schalingswetten voor de diffusie en viscositeit van lineaire ketens als functie van de moleculaire massa blijken in quantitatieve overeenstemming met experimentele observaties en theoretische voorspellingen. Deze resultaten onderstrepen dat de lengte van de lineaire polymeren en sterren die met dit model bestudeerd worden in het overgangsgebied liggen van het Rouse model naar reptatie-theorie. Analyses van de dynamische en reologische eigenschappen van smelten van ster-polymeren zijn gemaakt en vergeleken met met die van lineaire polymeren. Door de armlengte te vergroten, vertonen de ster-polymeren geleidelijk een tragere diffusie dan beide overeenstemmende lineaire polymeren, alsmede een vertraagde relaxatie. De verschillen tussen lineair en ster visco-elastisch gedrag kunnen ook worden gezien in hun opslag en verlies moduli; lineare ketens vertonen een bredere wig dan de overeenstemmende ster-polymeer na de kruisingsfrequentie van deze twee curves. Bovendien, in dit Hoofdstuk zijn de viscositeiten van ster smelten (in de limiet van oneindig trage afschuifstroming) berekend uit hun spanningsmoduli. De waargenomen exponentiële schalingswet met de moleculaire massa van de arm van het ster-polymeer vertoont een redelijke overeenkomst met experimentele en theoretische onderzoekingen voor zeer grote ster-polymeren beschreven in de literatuur.

In Hoofdstuk 4 worden asymmetrische ster-polymeren bediscussieerd waarvan één arm verschilt in lengte van de andere armen. We gebruiken de TWENTANGLEMENT techniek zoals beschreven in Hoofdstuk 2. Twee types asymmetrische sterren worden beschouwd: één heeft twee korte lineaire ruggengraat-armen en één langere asymmetrische arm; terwijl de ander twee relatief lange ruggengraat-armen en een korte asymmetrische arm bevat. Sructurele verschillen veroorzaakt door de lengte van de asymmetrische arm zijn bestudeerd door middel van hun autocorrelatiefuncties. Zoals verwacht, the langere arm vereist een langere relaxatietijd. De dynamica en ster relaxatie zijn verder bestudeerd door de vergelijking met overeenkomstige lineaire en symmetrische ster polymeren met vergelijkbare grootte. Door de lengte van asymmetrische armen te variëren vertonen de asymmetrische sterren een gedrag tussen die van de lineaire ketens en symmetrische sterren. Een duidelijkere trend wordt gezien in de relaxatie van asymmetrische sterren met twee lange ruggengraat armen; die sterren relaxeren veel trager dan alle overeenkomstige lineaire ketens. We verwachten betere statistische resultaten te vinden met een langere simulatietijd voor lineaire of ster polymeren met 
hogere moleculaire massa.

Het onderzoek van polymerensystemen op verschillende tijd- en lengteschalen vereist verschillende niveaus van grofkorreligheid. Hoofdstuk 5 presenteert een zeer grofkorrelig model, Responsive Particle Dynamics (RaPiD), waar elk polymeer is weergegeven door één deeltje. Krachten werken op de massamiddelpunten van elk polymeer. Deze grofkorrelige krachten kunnen worden gesplitst in conservatieve interacties beschreven door de uitgebreide Flory-Huggins potentiaal, "kortstondige" krachten afhankelijk van de contacten tussen polymeren om verknopingseffecten weer te geven, en willekeurige thermische krachten. In dit werk, in plaats van de 'top-down' (van boven naar beneden) aanpak die nu wordt gebruikt voor de parameterisering van $\mathrm{RaPiD}$, wordt de parametrisering verkregen in een 'bottom-up' (van onder naar boven) aanpak zowel als overwegingen van polymeerconcepten in de natuurkunde. Het Kremer-Grest model is gebruikt voor de atomaire of microscopische simulatie van drie-armige ster-polymeren met ongeveer één verknopingsmassa per arm. De radiëledistributiefunctie gegenereerd met RaPiD reproduceert de kleinschalige simulatiedata erg goed, wat er op wijst dat de uitgebreide Flory-Huggins potentiaal de statistische eigenschappen van dit ster-systeem zeer goed beschrijft. De diffusiecoefficient in RaPiD is gekozen door de wrijving per contact aan te passen. The afschuifspanning modulus zoals berekend in $\mathrm{RaPiD}$, zonder aanpassing van toegevoegde parameters, is in redelijke overeenstemming met de kleinschalige simulaties. Dit werk bewijst dat een veel-deeltjes model in staat is om de systeem dynamica zeer goed te beschrijven. Suggesties om deze overeenkomst te verbeteren worden gegeven aan het eind van dit Hoofdstuk . 



\section{Acknowledgment}

I have conceived of this moment many times in my mind, now it becomes reality in front of me. I believe it cannot be happened without support, patience, understanding and love, I am extremely grateful to all of you who share me with these.

First of all, I owe a debt of gratitude to my supervisor, Prof. Briels. Thank you Wim, for giving me the opportunity to become a CBPer, for leading me to the correct direction of my Ph.D. studies, for all your invaluable suggestions on important things. I would like to thank my assistant supervisor Dr. Wouter den Otter, for tackling the occurring problems and teaching on the research softwares. I want to say thank you to Dr. Johan Padding for introducing me the model with very helpful and supportive discussions.

I also would like to express my appreciation to Alessandra, Barry, Gabriel, Ioana, Igor, Tom, Matteo and Vishal, thank you for all your help and encouragement, especially in the period of preparing for my thesis, thanks for reading my manuscripts, giving me good suggestions and talking with me. There are also Csaba, Habi, Riccardo and Omar, I feel very happy to know you.

Abhi, Johannes, Phan Phi, and PG, my first ever foreign friends, thank you for the memorable Christmas dinner and the club nights we have spent with together. I want to say thank you to Xiaoming and Bowen, met @FOM conference, who both gave me very important encouragement when I doubted myself, Bowen thank you for your postcard from San Francisco. Liu Yi and Yuan Zhe, thank you for the dinner and I enjoy very much talking with you. Xiaoyue and Haizheng, I am glad to have you as my friends as well as the great neighbours. Lulu, Leilei and Hainan, I wish I could know you all earlier, times with you are very pleasant. Jinggang, Chao, Xiaokui, haishan, Yingzhe, Peng and Kenan, my brothers in Enschede, taking care of me and dealing with my troubles with no complaint, I owe you a big thanks.

Very special thanks to my parents, your endless love gives me the most powerful strength and your unlimited trust is the source of my courage. I also wish to thank my parents-in-law for their support and understanding. Last but not the least, I would like to take this opportunity to thank my beloved husband Shuwen. I am lucky to have you always support and encourage me aside. We have been through a very hard time, however it makes us even stronger. We 
will never forget that you can when you believe.

Here, I also want to appriciate my life in the past five years, during which I have grown up into 30+, I have enjoyed happiness and experienced difficulties, I have tasted sweet and sour... Probably I haven't figured it out absolutely, but I know it is not an end but a new start, this is life. 


\section{List of publications}

- Chapter 2: L. Liu, J.T. Padding, W.K. den Otter and W.J. Briels,

Coarse-grained simulations of moderately entangled star polyethylene melts,

J. Chem. Phys., 138, 244912, 2013.

- Chapter 3: L. Liu, W.K. den Otter and W.J. Briels,

Simulations of symmetric three-armed star polymers with different arm lengths, submitted to the Journal of Chemical Physics, 2014.

- Chapter 4: L. Liu, W.K. den Otter and W.J. Briels,

Dynamics of asymmetric star polymers under coarse-grained simulations,

in Manuscript.

- Chapter 5: L. Liu, W.K. den Otter and W.J. Briels,

Coarse Grain Forces in Star Polymer Melts,

submitted to Soft Matter, 2014. 



\section{Bibliography}

[1] J. M. G. Cowie. Polymers: Chemistry and Physics of modern materials. Blackie Academic \& Professional, London, U. K., 1991.

[2] R. G. Larson. The Structure and Rheology of Complex Fluids. Oxford University Press, Oxford, U. K., 1999.

[3] Mihaela Pascu Cornelia Vasile. Practical Guide to Polyethylene. Rapra Technology Limited, 2005.

[4] W. Pyckhout-Hintzen, J. Allgaier, and D. Richter. Recent developments in polymer dynamics investigations of architectually complex systems. European Polymer Journal, 47:474-485, 2011.

[5] Z. Wang, X. Chen, and R. G. Larson. Comparing tube models for predicting the linear rheology of branched polymer melts. J. Rheol., 54:223, 2010.

[6] D. J. Lohse, S. T. Milner, L. J. Fetters, and M. Xenidou. Well-defined, model long chain branched polyethylene. 2. melt rheological behavior. Macromolecules, 35:3066-3075, 2002.

[7] A. Jabbarzadeh, J. D. Atkinson, and R. I. Tanner. Effect of molecular shape on rheological properties in molecular dynamics simulation of star, h, comb, and linear polymer melts. Macromolecules, 36:5020, 2003.

[8] C. Baig, O. Alexiadis, and V. G. Mavrantzas. Advanced monte carlo algorithm for the atomistic simulation of short- and long-chain branched polymers: Implementation for model h-shaped, a3aa3 multiarm (pompom), and short-chain branched polyethylene melts. Macromolecules, 43:986, 2010.

[9] C. R. Bartels, Jr. B. Crist, L. J. Fetters, and W. W. Graessley. Self-diffusion in branched polymer melts. Macromolecules, 19:785, 1986.

[10] T. Cherdhirankorn, G. Floudas, H. J. Butt, and K. Koynov. Effects of chain topology on the tracer diffusion in star polyisoprenes. Macromolecules, 42:9183, 2009.

[11] K. Hur, C. Jeong, R. G. Winkler, N. Lacevic, R. H. Hee, and D. Y. Yoon. Chain dynamics of ring and linear polyethylene melts from molecular dynamics simulations. Macromolecules, 118:2451, 2011.

[12] L. J. Fetters, A. D. Kiss, and D. S. Pearson. Rheological behavior of star-shaped polymers. Macromolecules, 26:647, 1993.

[13] D. S. Pearson and E. Helfand. Viscoelastic properties of star-shaped polymers. Macromolecules, 17:888, 1984.

[14] W. W. Graessley and J. Roovers. Melt rheology of four-arm and six-arm star polystyrenes. Macromolecules, 12:959, 1979.

[15] M. Zamponi amd W. Pyckhout-Hintzen, A. Wischnewski, M. Monkenbusch, L. Willner, G. Kali, and D. Richter. Molecular observation of branch point motion in star polymer melts. Macromolecules, 43:518, 2010. 
[16] S. T. Milner and T. C. B. McLeish. Arm-length dependence of stress relaxation in star polymer melts. Macromolecules, 31:7479, 1998.

[17] C. H. Adams, L. R. Hutchings, P. G. Klein, T. C. B. McLeish, and R. W. Richards. Synthesis and dynamic rheological behavior of polybutadiene star polymers. Macromolecules, 29:5717, 1996.

[18] S. T. Milner and T. C. B. McLeish. Parameter-free theory for stress relaxation in star polymer melts. Macromolecules, 30:2159, 1997.

[19] J. T. Padding, E. van Ruymbeke, D. Vlassopoulos, and W. J. Briels. Computer simulation of the rheology of concentrated star polymer suspensions. Rheol. Acta, 49:473-484, 2010

[20] N. Ch. Karayiannis and V. G. Mavrantzas. Hierarchical modeling of the dynamics of polymers with a nonlinear molecular architecture: calculation of branch point friction and chain reptation time of h-shaped polyethylene melts from long molecular dynamics simulations. Macromolecules, 38:8583, 2005.

[21] Nikos Ch. Karayiannis, Ageliki E. Giannousaki, and Vlasis G. Mavrantzas. An advanced monte carlo method for the equilibration of model long-chain branched polymers with a well-defined molecular architecture: Detailed atomistic simulation of an h-shaped polyethylene melt. J. Chem. Phys., 118:2451-2454, 2003.

[22] Glen H. Kirby and Jennifer A. Lewis. Comb polymer architecture effects on the rheological property evolution of concentrated cement suspensions. J. Am. Ceram. Soc., 87:16431652, 2008

[23] D. R. Daniels, T. C. B. McLeish, B. J. Crosby, R. N. Young, and C. M. Fernyhough. Molecular rheology of comb polymer melts. 1. linear viscoelastic response. Macromolecules, 34:70257033, 2001.

[24] E. van Ruymbeke, K. Orfanou, M. Kapnistos, H. Iatrou, M. Pitsikalis, N. Hadjichristidis, D. J. Lohse, and D. Vlassopoulos. Entangled dendritic polymers and beyond: rheology of symmetric cayley-tree polymers and macromolecular self-assemblies. Macromolecules, 40:59415952, 2007.

[25] V. A. Harmandaris, V. G. Mavrantzas, D. N. Theodorou, M. Kröger, J. Ramírez, H. C. Öttinger, and D. Vlassopoulos. Crossover from the rouse to the entangled polymer melt regime: Signals from long, detailed atomistic molecular dynamics simulations, supported by rheological experiments. Macromolecules, 36:1376, 2003 .

[26] K. Kremer, G. S. Grest, and I. Carmesin. Crossover from rouse to reptation dynamics: a molecular-dynamics simulation. Phys. Rev. Lett., 61:566, 1988

[27] D. S. Pearson, G. Ver Strate, E. Von Meerwall, and F. C. Schilling. Viscosity and self-diffusion coefficient of linear polyethylene. Macromolecules, 20:1133-1141, 1987.

[28] D. S. Pearson, L. J. Fetters, and W. W. Graessley. Viscosity and self-diffusion coefficient of hydrogenated plybutadiene. Macromolecules, 27:711, 1994.

[29] S. Onogi, T. Masuda, and K. Kitagawa. Rheological properties of anionic polystyrenes. i. dynamic viscoelasticity of narrow-distributution polystyrenes. Macromolecules, 3:109, 1969.

[30] J. Liu, W. Yu, and C. Zhou. Polymer chain topological map as determined by linear viscoelasticity. J. Rheol., 55:545, 2011. 
[31] C. B. Gell, W. W. Graessley, and L. J. Fetters. Viscoelasticity and self-diffusion in melts of entangled linear polymers. J. Polym. Sci. B Polym. Phys., 35:1933, 1997.

[32] J. D. Moore, S. T. Cui, H. D. Cochran, and P. T. Cummings. A molecular dynamics study of a short-chain polyethylene melt.: I. steady-state shear. J. Non-Newtonian Fluid Mech., 93:83, 2000.

[33] W. Paul, G. D. Smith, and D. Y. Yoon. Static and dynamic properties of a n-c100h202 melt from molecular dynamics simulations. Macromolecules, 30:7772, 1997.

[34] P.E.Rouse. A theory of the linear viscoelastic properties of dilute solutions of coiling polymers. J. Chem. Phys., 21:1272, 1953.

[35] M. Doi and S. F. Edwards. The Theory of Polymers Dynamics. Oxford Science Publications, Oxford, U. K., 1986.

[36] P. G. de Gennes. Scaling Concepts in Polymer Physics. Cornell University Press, Ithaca, New York, 1979.

[37] Iwao Teraoka. Polymer Solutions: An Introduction to Physical Properties. A John Wiley \& Sons, Inc., Publication, Brooklyn, New York, 2002.

[38] M. P. Allen and D. J. Tildesley. Computer Simulations of Liquids. Oxford University Press, Oxford, U. K., 1987.

[39] H. C. Öttinger. Stochastic Processes in Polymeric Fluids - Tools and Examples for Developing Simulation Algorithms. Springer, Berlin, Germany, 1996.

[40] F. Müller-Plathe. Coarse-graining in polymer simulation: From the atomistic to the mesoscopic scale and back. Chem. Phys. Chem., 3:754, 2002.

[41] J. T. Padding and W. J. Briels. Systematic coarse-graining of the dynamics of entangled polymer melts: the road from chemistry to rheology. J. Phys. Condens. Matter, 23:233101, 2011.

[42] T. Strauch, L. Yelash, and W. Paul. A coarse-graining procedure for polymer melts applied to 1,4polybutadiene. Phys. Chem. Chem. Phys., 11:1942-1948, 2009.

[43] K. Kremer and G. S. Grest. Dynamics of entangled linear polymer melts: A molecular-dynamics simulation. J. Chem. Phys, 92:5057, 1990.

[44] J. T. Padding and W. J. Briels. Uncrossability constraints in mesoscopic polymer melt simulations: Nonrouse behavior of c120h242. J. Chem. Phys., 115:2846, 2001.

[45] J. T. Padding and W. J. Briels. Time and length scales of polymer melts studied by coarse-grained molecular dynamics simulations. J. Chem. Phys., 117:925, 2002.

[46] L. Liu, J. T. Padding, W. K. den Otter, and W. J. Briels. Coarse-grained simulations of moderately entangled star polyethylene melts. J. Chem. Phys., 138:244912, 2013.

[47] R. Prez-Aparicio, J. Colmenero, F. Alvarez, J.T. Padding, and W.J. Briels. Chain dynamics of poly(ethylenealt-propylene) melts by means of coarse-grained simulations based on atomistic molecular dynamics. $J$. Chem. Phys., 132:024904, 2010.

[48] I. S. Santos de Oliveira, B. W. Fitzgerald, W. K. den Otter, and W. J. Briels. Mesoscale modeling of shearthinning polymer solutions. J. Chem. Phys., 140:104903, 2014. 
[49] R. L. C. Akkermans and W. J. Briels. A structure-based coarse-grained model for polymer melts. J. Chem Phys., 114:1020, 2001.

[50] W. J. Briels. Transient forces in flowing soft matter. Soft Matter, 5:4401, 2009.

[51] P. Kindt and W. J. Briels. A single particle model to simulate the dynamics of entangled polymer melts. $J$. Chem. Phys., 127:134901, 2007.

[52] J. Sprakel, J. T. Padding, and W. J. Briels. Transient forces and non-equilibrium states in sheared polymer networks. Europhys. Lett., 93:58003, 2011.

[53] A. van den Noort, W. K. den Otter, and W. J. Briels. Coarse graining of slow variables in dynamic simulations of soft matter. Europhys. Lett., 80:28003, 2007.

[54] A. van den Noort and W. J. Briels. Coarse-grained simulations of elongational viscosities, superposition rheology and shear banding in model core-shell systems. Macromol. Theory Simul., 16:742-754, 2007.

[55] A. van den Noort and W. J. Briels. Brownian dynamics simulations of concentration coupled shear banding. J. Non-Newtonian Fluid Mech., 152:148-155, 2008.

[56] I. S. Santos de Oliveira, A. van den Noort, J. T. Padding, W. K. den Otter, and W. J. Briels. Alignment of particles in sheared viscoelastic fluids. J. Chem. Phys., 135:104902, 2011.

[57] I. S. Santos de Oliveira, W. K. den Otter, and W. J. Briels. The origin of flow-induced alignment of spherical particles in shear-thinning viscoelastic fluids. accepted for publication on the J. Chem. Phys., 137:204908, 2012.

[58] J. T. Padding, L. V. Mohite, D. Auhl, W. J. Briels, and C. Bailly. Mesoscale modeling of the rheology of pressure sensitive adhesives through inclusion of transient forces. Soft Matter, 7:5036, 2011.

[59] W. J. Briels. Theory of Polymer Dynamics, lecture notes. It can be downloaded from: http://cbp.tnw.utwente.nl/PolymeerDictaat, Enschede, The Netherlands, 1998.

[60] J. L. Barrat and J. P. Hansen. Basic Concepts for Simple and Complex Liquids. Cambridge University Press, UK, 2003.

[61] L. J. Fetters, A. D.Kiss, and D. S. Pearson. Rheological behaviors of star-shaped polymers. Macromolecules, 26:647, 1993.

[62] Tom C. B. McLeish and Scott T. Milner. Entangled dynamics and melt flow of branched polymers. Adv. in Polym. Sci., 143:195, 1999.

[63] M. Doi and S. F. Edwards. The Theory of Polymer Dynamics. Oxford:Clarendon, 1986.

[64] D. S. Pearson, G. Ver Strate, E. Von Meerwall, and F. C. Schilling. Viscosity and self-diffusion coefficient of linear polyethylene. Macromolecules, 20:1133-1141, 1997.

[65] S. Q. Wang. Chain dynamics in entangled polymers: Diffusion versus rheology and their comparison. $J$. Polym. Sci. Part B Polym. Phys., 41:1589, 2003.

[66] V. A. Harmandaris, V. G. Mavrantzas, and D. N. Theodorou. Atomistic molecular dynamics simulation of polydisperse linear polyethylene melts. Macromolecules, 31:7934, 1998. 
[67] Y. L. Zhu, H. Liu, and Z. Y. Lu. A highly coarse-grained model to simulate entangled polymer melts. J. Chem. Phys., 136:144903, 2012.

[68] G. Ianniruberto and G. Marrucci. Entangled melts of branched ps behave like linear ps in the steady state of fast elongational flows. Macromolecules, 46:267, 2013.

[69] Y. Masubuchi, G. Ianniruberto, F. Greco, and G. Marrucci. Entanglement molecular weight and frequency response of sliplink networks. J. Chem. Phys., 119:6925, 2003.

[70] Y. Masubuchi, J. Takimoto, K. Koyama, G. Ianniruberto, G. Marrucci, and F. Greco. Brownian simulations of a network of reptating primitive chains. J. Chem. Phys., 115:4387, 2001.

[71] C. C. Hua and J. D. Schieber. Segment connectivity, chain-length breathing, segmental stretch, and constraint release in reptation models. i. theory and single-step strain predictions. J. Chem. Phys., 109:10018, 1998.

[72] D. M. Nair and J. D. Schieber. Linear viscoelastic predictions of a consistently unconstrained brownian slip-link model. Macromolecules, 39:3386, 2006

[73] A. E. Likhtman. Single-chain slip-link model of entangled polymers: Simultaneous description of neutron spin-echo, rheology, and diffusion. Macromolecules, 38:6128, 2005.

[74] R. S. Graham, A. E. Likhtman, T. C. B. McLeish, and S. T. Milner. Microscopic theory of linear, entangled polymer chains under rapid deformation including chain stretch and convective constraint release. J. Rheol., 47:1171, 2003.

[75] A. E. Likhtman1, S. T. Milner, and T. C. B. McLeish. Microscopic theory for the fast flow of polymer melts. Phys. Rev. Letters, 85:4550, 2000.

[76] B. H. Zimm and W. H. Stockmayer. The dimensions of chain molecules containing branches and rings. $J$. Chem. Phys., 17:1301, 1949.

[77] N. Hadjichristidis et al., D. J. Lohse et al., R. A. Mendelson, T. Sun, and C. J. Ruff. Well-defined, model long chain branched polyethylene. 1. synthesis and characterization. Macromolecules, 33:2424, 2000.

[78] G. Kraus and J. T. Gruver. Rheological properties of multichain polybutadienes. J. Polym. Sci., A3:105122, 1965.

[79] C. R. Bartels, Jr. B. Crist, L. J. Fetters, and W. W. Graessley. Self-diffusion in branched polymer melts. Macromolecules, 19:785, 1986.

[80] P. G. de Gennes. Reptation of stars. J. Phys. France, 36:1199, 1975.

[81] M. Doi and N. Y. Kuzuu. Rheology of star polymers in concentrated solutions and melts. J. Polym. Sci., Polym. Lett. Ed., 18:775, 1980.

[82] D. S. Pearson and E. Helfand. Viscoelastic properties of star-shaped polymers. Macromolecules, 17:888, 1984.

[83] S. T. Milner and T. C. B. McLeish. Arm-length dependence of stress relaxation in star polymer melts. Macromolecules, 31:7479, 1998. 
[84] R. C. Ball and T. C. B. McLeish. Dynamic dilution and the viscosity of star-polymer melts. Macromolecules, 22:1911, 1989.

[85] J. Klein, D. Fletcher, and L.J. Fetters. Diffusional behavior of entangled star polymers. Nature, 304:526, 1983.

[86] K. R. Shull, E. J. Kramer, and L. J. Fetters. Effect of number of arms on diffusion of star polymers. Nature, 345:790, 1990.

[87] I. Prigogine, Stuart A. Rice, Gary S. Grest, Lewis J. Fetters, John S. Huang, and Dieter Richter. Star polymers: Experiment theory and simulation. Advances in Chemical Physics: Polymeric Systems, 94, 2007.

[88] S. T. Milner and T. C. B. McLeish. Parameter-free theory for stress relaxation in star polymer melts. Macromolecules, 30:2159, 1997.

[89] D. J. Read, D. Auhl, C. Das, J. den Doelder, M. Kapnistos, I. Vittorias, and T. C. McLeish. Linking models of polymerization and dynamics to predict branched polymer structure and flow. Science, 333:1871, 2011.

[90] C. Das, N. J. Inkson, D. J. Read, M. A. Kelmanson, and T. C. B. McLeish. Computational linear rheology of general branch-on-branch polymers. Journal of Rheology, 50:207-234, 2006.

[91] R. G. Larson. Combinatorial rheology of branched polymer melts. Macromolecules, 34:4556, 2001.

[92] V. A. Harmandaris, V. G. Mavrantzas, and D. N. Theodorou. Atomistic molecular dynamics simulation of polydisperse linear polyethylene melts. Macromolecules, 31:7934, 1998.

[93] K. Kremer and G. S. Grest. Dynamics of entangled linear polymer melts: A molecular-dynamics simulation. J. Chem. Phys, 92:5057, 1990.

[94] G. S. Grest, K. Kremer, S. T. Milner, and T. A. Witten. Relaxation of self-entangled many-arm star polymers. Macromolecules, 22:1904, 1989.

[95] G. S. Grest, K. Kremer, and T. A. Witten. Structure of many arm star polymers: a molecular dynamics simulation. Macromolecules, 20:1987, 1987.

[96] Q. Zhou and R. G. Larson. Direct molecular dynamics simulation of branch point motion in asymmetric star polymer melts. Macromolecules, 40:3443, 2007.

[97] P. Bačová, L.G.D. Hawke, D.J. Read, and A.J. Moreno. Dynamics of branched polymers: A combined study by molecular dynamics simulations and tube theory. Macromolecules, 46:4633, 2013.

[98] S. Shanbhag and R. G. Larson. A slip-link model of branch-point motion in entangled polymers. Macromolecules, 37:8160, 2004.

[99] Y. Masubuchi, T. Yaoita, Y. Matsumiya, and H. Watanabe. Primitive chain network simulations for asymmetric star polymers. J. Chem. Phys., 134:194905, 2011.

[100] I. Carmesin and K. Kremer. The bond fluctuation method: a new effective algorithm for the dynamics of polymers in all spatial dimensions. Macromolecules, 21:4219, 1988.

[101] J. Scott Shaffer. Effects of chain topology on polymer dynamics: Bulk melts. J. Chem. Phys., 101:4205, 1994. 
[102] Tadeusz Pakula. Static and dynamic properties of computer simulated melts of multiarm polymer stars. Computational and Theoretical Polymer Science, 8:21, 1998.

[103] T. Pakula, D. Vlassopoulos, G. Fytas, and J. Roovers. Structure and dynamics of melts of multiarm polymer stars. Macromolecules, 31:8931, 1998.

[104] S. Brown and G. Szamel. Computer simulaion of three-arm star polymers. Macromol. Theory Simul., 9:14, 2000 .

[105] D. Reith, M. Pütz, and F. Müller-Plathe. Deriving effective mesoscale potentials from atomistic simulations. J. Comput. Chem., 24:1624, 2003.

[106] M. Mondello, G. S. Grest, E. B. Webb III, and P. Peczak. Dynamics of $n$-alkanes: Comparison to rouse. $J$. Chem. Phys, 109:798, 1998 .

[107] M. Mondello and G. S. Grest. Viscosity calculation of $n$-alkanes by equilibrium molecular dynamics. $J$. Chem. Phys, 106:9327, 1997.

[108] M. Kapnistos, G. Koutalas, N. Hadjichristidis, J. Roovers, D. J. Lohse, and D. Vlassopoulos. Linear rheology of comb polymers with star-like backbones: melts and solutions. Rheol. Acta, 46:273, 2006.

[109] H. Watanabe. Viscoelasticity and dynamics of entangled polymers. Progr. in Polym. Sci., 24:1253, 1999.

[110] T. C. B. McLeish. Tube theory of entangled polymer dynamics. Advances in Physics, 51:1379, 2002.

[111] G. Quack and L.J. Fetters. Rheological properties of multichain polybutadienes. J. Polym. Sci., A3:105122, 1977.

[112] S. J. Park, S. Shanbhag, and R. G. Larson. A hierarchical algorithm for predicting the linear viscoelastic properties of polymer melts with long-chain branching. Rheol. Acta, 44:319, 2005.

[113] X. Chen and R. G. Larson. Effect of branch point position on the linear rheology of asymmetric star polymers. Macromolecules, 41:6871, 2008.

[114] J. T. Padding and W. J. Briels. Zero-shear stress relaxation and long time dynamics of a linear polyethylene melt: A test of rouse theory. J. Chem. Phys., 114:8685, 2001.

[115] Y. Li, B. C. Abberton, and M. Kröger. Challenges in multiscale modeling of polymer dynamics. Polymers, $5: 751,2013$.

[116] S. D. Anogiannakis, C. Tzoumanekas, and D. N. Theodorou. Microscopic description of entanglements in polyethylene networks and melts:strong, weak, pairwise, and collective attributes. Macomolecules, 45:9475, 2012.

[117] V. A. Harmandaris and K. Kremer. Dynamics of polystyrene melts through hierarchical multiscale simulations. Macromolecules, 42:791, 2009.

[118] Y. Masubuchi, J. I. Takimoto, K. Koyama, G. Ianniruberto, G. Marrucci, and F. Greco. Brownian simulations of a network of reptating primitive chains. J. Chem. Phycs., 115:4387, 2001.

[119] R. B. Bird, R. C. Armstrong, and O. Hassager. Dynamics of Polymeric Liquids, Volume 1, Fluid Mechanics, 2nd Edition. John Wiley \& Sons, Inc., United states of America, 1987. 
[120] V. Schmitt, C. M. Marques, and F. Lequeux. Shear-induced phase separation of complex fluids: The role of flow-concentration coupling. Phys. Rev. E, 52:4009, 1995.

[121] J. K. G. Dhont, K. Kang, M. P. Lettinga, and W. J. Briels. Shear-banding instabilities. Korea-Australia Rheology Journal, 22:291, 2010.

[122] A. van den Noort, W. K. den Otter, and W. J. Briels. Coarse graining of slow variables in dynamic simulations of soft matter. Europhys. Lett., 80:28003, 2007.

[123] I. Santos de Oliveira, A. van den Noort, J. T. Padding, W. K. den Otter, and W. J. Briels. Alignment of particles in sheared viscoelastic fluids. J. Chem. Phys., 135:104902, 2011.

[124] I. Santos de Oliveira, W. K. den Otter, and W. J. Briels. Alignment and segregation of bidisperse colloids in a shear-thinning viscoelastic fluid under shear flow. EuroPhys. Lett., 101:28002, 2013.

[125] J.T. Padding, L.V. Mohite, D. Auhl, T. Schweizer, W.J. Briels, and C. Bailly. Quantitative mesoscale modeling of the oscillatory and transient shear rheology and the extensional rheology of pressure sensitive adhesives. Soft Matter, 8:7967, 2012.

[126] G. J. Schneider, K. Nusser, S. Neueder, M. Brodeck, L. Willner, B. Farago, O. Holderer, W. J. Briels, and Dieter Richter. Anomalous chain diffusion in unentangled model polymer nanocomposites. Soft Matter, 9:4336, 2013

[127] I. Santos de Oliveira, B. Fitzgerald, W. K. den Otter, and W. J. Briels. Mesoscale modeling of shear-thinning polymer solutions. J. Chem. Phys., 140:104903, 2014.

[128] R. L. C. Akkermans and W. J. Briels. Coarse-grained dynamics of one chain in a polymer melt. J. Chem. Phys., 113:6409, 2000.

[129] C. N. Likos. Effective interactions in soft condensed matter physics. Physics Reports, 348:267, 2001.

[130] C. N. Likos, H. Löwen, M. Watzlawek, B. Abbas, O. Jucknischke, J. Allgaier, and D. Richter. Star polymers viewed as ultrasoft colloidal particles. Physical Review Letters, 80:4450, 1998.

[131] A. Jusuf, J. Dzubiella, C. N. Likos, C. von Ferber, and H. Löwen. Effective interactions between star polymers and colloidal particles. Journal of Physics: Condensed Matter, 13:6177, 2001.

[132] F. Schmid. Self-consistent-field theories for complex fluids. J. Physics-Condensed Matter, 10:8105, 1998.

[133] I. Pagonabarraga and D. Frenkel. Dissipative particle dynamics for interacting systems. J. Chem. Phys., $115: 5015,2001$

[134] S. Y. Trofimov, E. L. F. Nies, and M. A. J. Michels. Thermodynamic consistency in dissipative particle dynamics simulations of strongly nonideal liquids and liquid mixtures. J. Chem. Phys., 117:9383, 2002.

[135] J. M. Deutch and I. Oppenhei. Molecular theory of brownian motion for several particles. J. Chem. Phys., $54: 3547,1971$.

[136] D. L. Ermak and J. A. McCammon. Brownian dynamics with hydrodynamic interactions. J. Chem. Phys., 69:1352, 1978.

[137] S. Kjelstrup, D. Bedeaux, E. Johannessen, and J. Gross. Non-Equilibrium Thermodynamics for Engineers. World Scientific, 2010. 
[138] J. Sprakel, E. Spruijt, J. van der Gucht, J. T. Padding, and W. J. Briels. Failure-mode transition in transient polymer networks with particle-based simulations. Soft Matter, 5:4748, 2009.

[139] W. J. Briels and H. L. Tepper. Crystal growth of the lennard-jones (100) surface by means of equilibrium and nonequilibrium molecular dynamics. Physical Review Letters, 79:5074, 1997.

[140] D. Chandler. Introduction to Modern Statistical Mechanics. Oxford University Press, USA, 1987.

[141] B. Hess and C. Kutzner and D. van der Spoel and E. Lindahl. Gromacs 4: algorithms for highly efficient, load-balanced, and scalable molecular simulation. J. Chem. Theory Comput., 4:435, 2008. 
